\title{
Dynamics of an Epidemic Model under the Influence of Environmental Stress Sangeeta Saha*, Guruprasad Samanta**
}

Department of Mathematics, Indian Institute of Engineering Science and Technology, Shibpur, Howrah - 711103, India

\begin{abstract}
We have considered a compartmental epidemiological model with infectious disease to observe the influence of environmental stress on disease transmission. The proposed model is well-defined as the population at each compartment remains positive and bounded with time. Dynamical behaviour of the model is observed by the stability and bifurcation analysis at the equilibrium points. Also, numerical simulation supports the theoretical proofs and the result shows that the system undergoes a forward bifurcation around the disease-free equilibrium. Our results indicate that with the increase of environmental pollution, the overall infected population increases. Also, the disease transmission rate among the susceptible and stressed population from asymptomatically infected individuals plays a crucial role to make a system endemic. A corresponding optimal control problem has also been proposed to control the disease prevalence as well as to minimize the cost by choosing the vaccination policy before being infected and treatment policy to the infected as control variables. Numerical figures indicate that the vaccination provided to susceptible needs some time to reduce the disease transmission but the vaccination provided to stressed individuals works immediately after implementation. The treatment policy for symptomatically infected individuals works with a higher rate at an earlier stage but the intensity decreases with time. Simultaneous implementation of all control interventions is more useful to reduce the size of overall infective individuals and also to minimize the economic burden. Hence, this literature clearly expresses the impact of environmental pollution (specifically the influence of environmental stress) on the disease transmission in the population.
\end{abstract}

Key words: environmental stress, sensitivity analysis, stability, optimal control.

\section{INTRODUCTION}

In the continuous changing world, there are a variety of stresses from toxins to the vicissitudes of climate change present in the environment which may affect the organisms. The presence of environmental stress enhances the effects of pollutants on organisms. The impact of these stress depends on the dynamical behaviour of the corresponding stressed system. One of the interesting areas is to analyse the combined impacts of environmental stress and infectious diseases. There are many reports indicating that infectious diseases play a key role in the dynamics of many natural populations [1]. So, one can assume that environmental stress either aggravates or mitigates the transmission of infectious disease among susceptible, depending on the aspects of the system which are emphasized.

Let us consider the interplay of disease and stress from the perspective of an individual host which is affected by a host-specific parasite. It is expensive to maintain the immune systems and

\footnotetext{
*sangeetasaha629@gmail.com

**Corresponding author: g_p_samanta@yahoo.co.uk, gpsamanta@math.iiests.ac.in
} 
so, stressed animals may fail to produce enough energy to fulfil a beneficial defence [2]. There are many stressors present, like malnutrition []ㅡ, toxic chemicals in environmental pollution [4], thermal stress due to climate change [5] etc. which increase the probability of getting infected from susceptible through infectious diseases. As a conclusion, it is stated that stressed individuals are more susceptible to infection $[6,1]$. It is evident that within a species, the impact of any stressor varies with genotype [7]. Hence, environmental stresses escalate the transmission of an infectious disease.

Environmental pollution has become a concerning topic these days. It contains water pollution, air pollution as well as soil pollution. The toxic chemicals, present in the environment, can be transmitted to the population by water, air and food. Most of these chemicals make a harmful effect on living species. A lot of work has been done analysing the perilous impact of this pollution on ecosystem $[\underline{8}, \underline{9}, \underline{10}]$. Environmental pollution causes several other diseases including respiratory and cardiovascular diseases. Research leads to the conclusion that increasing environmental pollution create difficulties in the way of survival of animal and plant population.

Growing industrialization causes the air to be highly contaminated by gaseous pollutants and particulate matters like $\mathrm{PM}_{10}$ and $\mathrm{PM}_{2.5}$. Also, there are natural activities like volcanoes, forest fire, and anthropogenic activities etc. which increase the damage to the environment as the air pollutants containing poisonous gases (e.g. $\mathrm{SO}_{2}$, $\mathrm{CO}$, $\mathrm{NO}$ etc.), hydrocarbons, metals (mercury, lead etc.) as well as particulate matters $\left(\mathrm{PM}_{2.5}, \mathrm{PM}_{10}\right)$ increase for these. Moreover, there are some organic pollutants too, for example, pesticides, dioxin etc. which form toxic chemicals.

The mentioned air pollutants can even damage the signalling pathways of hormones and reproductive system [11], cause dysfunction of tissue due to shortage of oxygen (ischaemia), create an irregularity in heartbeats (arrhythmias) [12], premature mortality [13], lung cancer, cardiovascular disease mortality, cardiac problems [14] etc. Javan et al. have conducted a two-year experiment in 2013 to observe the effects of exposure particulate matter $\left(\mathrm{PM}_{10}\right)$ at Zabol in Iran [15]. They have concluded that the total mortality, cardiovascular mortality, respiratory mortality etc. are increased by almost $50 \%$ in 2014-2015 and this situation needs some urgent control actions. There are some research works revealing the relationship between biomass fuels (BMF) and traffic-related air pollution (TRAP) and among those works, some consider the impact of the infectious diseases like tuberculosis, asthma etc. [16] in their system. Moreover, several studies reveal that air pollution makes a negative impact on newborns and children [17]. It is observed that the air pollutants affect the developing embryos [18] and newborn causing increased systolic blood pressure (SBP) [19].

Scarcity of safe drinking water becomes a concerned topic at present. Wastage from different industries and agricultural activities are the main contributors which contaminate various water sources. Now, there is water bactericide which is used for water treatment but these disinfectants have some side effects which make negative impacts. Almost 100,000 water contaminants are registered in the water which is used regularly [20]. Low birth weight, spontaneous abortions, pre-term delivery, stillbirth, birth defects and lung cancer, adverse reproductive and developmental defects etc. are some of the examples of noxious impacts of the consequences of disinfectants $[21, \underline{22}, \underline{23}]$. So, exposure of pregnant women to contaminated water may have risky side-effects $[\underline{24}, \underline{25}, \underline{26}]$.

There are some researches which reveal that the development of the immune system may be impeded due to the exposure to different pollutants [27, 28, 29]. As per the work performed by Hertz-Picciotto et al. [27] it may be concluded that impact of some organic compounds are proved to be more dangerous in the prenatal period and early exposure to these chemicals increases the risk of infection significantly [28]. Raqib et al. [29] collected data of those pregnant women from a rural area in Bangladesh who were affected with arsenic contamination, to make 
their experiment to study the impact of arsenic exposure on the development of the immune system. They concluded that arsenic exposure causes increased morbidity.

Research reveals that climate changes and an increase in temperature due to emission of pollutants and greenhouse gases (because of anthropogenic activities) have made negative impacts on the environment $[30, \underline{31}, \underline{32}, \underline{33}, \underline{34}, \underline{35}, \underline{36}$. Several studies have been done to analyse the impact of environmental pollution on disease transmission [32, 37]. Lipp et al. [32], in their studies proved that the emitted pollutants due to the anthropogenic activities increase the normal temperature and even change the UV intensity. They concluded that this change in temperature would expand the prevalence of V. cholerae. The work done by Patz et al. is dealt with the impact of environmental changes due to environmental pollution on various infectious diseases [31].

There are certain infectious diseases in which we get individuals who can transmit their illness without showing any symptoms. These individuals said to be "carriers", play an important role in the disease transmission. Asymptomatic individuals are those who unaware of their illness and hence can infect others easily. Typhoid fever caused by the bacteria Salmonella Typhi is an infectious disease where in some cases people do not exhibit any symptoms for a long time. At the beginning of the $20^{\text {th }}$ century people came to know about this fever from the cases of Typhoid Mary in the US and "Mr. N the milker" in England. In that case, the asymptomatically infected individuals transmitted disease among many people over the years at workplaces as well as at homes. Even today, almost 21 million people are getting infected and approximately 200,000 people are died by Typhoid fever worldwide each year. Asymptomatic individuals are considered to play a vital role in the transmission of Typhi bacteria which is an obligation for the eradication of Typhoid fever using vaccination and treatment [38].

Let us take another major infectious disease hepatitis B in which long-term asymptomatic carriage is observed. It is a liver disease caused by the HBV virus from the Hepadnavirus family. In most of the cases, the infected people completely recover after proper treatment and develop lifetime immunity. Several reports say that almost 5-10\% of adults suffer from chronic HBV infection, and about 15-25\% of them develop the liver disease later. Jaundice, nausea, abdominal pain, joint pain, fatigue etc. are some of the basic symptoms of Hepatitis B. But approximately $30 \%$ of infected people do not show any of the mentioned symptoms. The existence of a large proportion of chronic asymptomatic individuals hinders to control hepatitis B infection in many countries as they can transmit most of the infections. There are many other infectious diseases where infected people go through this asymptomatic stage. Further, there is another virus named, Epstein-Barr Virus (EBV) of the herpes family which causes infectious mononucleosis, known as glandular fever. Most of the infected people remain in an asymptomatic stage in this case as there do not any symptoms developed but the virus remains dormant in the cells of the throat and the immune system for the rest of their lives. Further, Clostridium difficile associated disease (CDAD) occurs from Clostridium difficile bacillus. The disease cause diarrhoea, toxic mega-colon, pseudo-membranous colitis, perforation of the colon etc. More than 300,000 cases of diarrhoea have been reported yearly in the United States and up to $30 \%$ of asymptomatic infected have been reported in long-term care facilities. So, it can be assumed that asymptomatically infected people may propagate the disease transmission causing large outbreaks of CDAD in North America and Europe [39].

Rest of the paper has been organized as follows: in the following section, we have formulated a compartmental epidemiological model to analyse the impact of environmental stress on the disease transmission. A new compartment has been introduced where individuals are considered to be affected by environmental pollution and hence have a larger chance to become infected. Section 3 shows that the model is well-posed as the state variables are positive and bounded over time. In Section 4, equilibrium analysis with basic reproduction number has been performed. 
Section 5 analyses the sensitivity of different parameters in $R_{0}$. Section 6 deals with the stability conditions of the equilibrium points. In Section 7, it has been shown that the system undergoes a transcritical bifurcation at $R_{0}=1$. Section 8 contains the pictorial scenarios of the system dynamics without applying any control strategies. In section 9, control interventions have been implemented to control the infection. The subsequent section contains the numerical simulations for the proposed control system and the last section includes a brief conclusion.

\section{MATHEMATICAL MODEL: BASIC EQUATIONS}

We have formulated a five compartmental epidemic model to analyse the effect of environmental pollution on the spread of infectious diseases mainly caused by air or water pollution or even radioactive contamination. Let us consider the total population as $N(t)$ which is subdivided into five phases at any time $t$, i.e., susceptible population $X_{1}(t)$, portion of susceptible population which is affected by environmental pollution and moved into stressed population $X_{2}(t)$, asymptomatically infected population $I_{1}(t)$, symptomatically infected population $I_{2}(t)$ and recovered population $R(t)$. Natural recruitment rate of total susceptible population is taken as a constant $\Lambda$, out of which, a proportion $p$ of them enters directly into stressed class $X_{2}(t)$ and remaining $(1-p)$ stays in $X_{1}(t)$ class. Now, the susceptible individuals in $X_{1}$ class move to the stressed class with a rate of $\theta$ because regular exposure to environmental pollution is considered here. At every stage, the natural death rate of human populations is $d$ whereas the disease-related death rate is given by $\delta$ and it has been taken only in two infected stages.

A susceptible population can become infected (asymptomatically) after getting touch with both asymptomatically and symptomatically infected population with a rate of $\beta_{1}$ and $\beta_{2}$ respectively. It is evident that the stressed population can be infected more easily and so, it is assumed that the disease transmission rates are higher for the stressed population than the susceptible population. Considering this fact, we have introduced some stress-related parameters $\omega_{1}, \omega_{2}$ and $\beta_{1}^{\prime}, \beta_{2}^{\prime}$, where, $\omega_{1}, \omega_{2}$ denote the levels of stress while $\beta_{1}^{\prime}, \beta_{2}^{\prime}$ represent the extents to which stress can be increased for $\beta_{1}$ and $\beta_{2}$ respectively [40]. Hence, both the transmission rates for individuals of $X_{2}$ class is modified as $\beta_{1}\left(1+\omega_{1} \beta_{1}^{\prime}\right)$ and $\beta_{2}\left(1+\omega_{2} \beta_{2}^{\prime}\right)$ respectively . The asymptomatically infected individuals move to the symptomatic phase at a rate of $\eta$ while on the other hand, symptomatically infected individuals are moved to recovered class at a constant rate of $\alpha$. It is assumed that the recovered people can move back to susceptible and stressed class due to the presence of environmental pollution with a rate of $\gamma$. So, a proportion $q$ of recovered population joins the $X_{2}(t)$ class and the remaining fraction $(1-q)$ enters into the $X_{1}(t)$ class. A schematic diagram has been provided in Figure 1 to get a better insight into the proposed model.

Considering all the assumptions and taking all the parameters as a positive quantity, we get our system as:

$$
\begin{aligned}
\frac{d X_{1}}{d t} & =(1-p) \Lambda-d X_{1}-\theta X_{1}+(1-q) \gamma R-\left(\beta_{1} I_{1}+\beta_{2} I_{2}\right) X_{1}, \quad X_{1}(0)>0, \\
\frac{d X_{2}}{d t} & =p \Lambda+\theta X_{1}-d X_{2}+q \gamma R-\left\{\beta_{1}\left(1+\omega_{1} \beta_{1}^{\prime}\right) I_{1}+\beta_{2}\left(1+\omega_{2} \beta_{2}^{\prime}\right) I_{2}\right\} X_{2}, \quad X_{2}(0)>0, \\
\frac{d I_{1}}{d t} & =\left(\beta_{1} I_{1}+\beta_{2} I_{2}\right) X_{1}+\left\{\beta_{1}\left(1+\omega_{1} \beta_{1}^{\prime}\right) I_{1}+\beta_{2}\left(1+\omega_{2} \beta_{2}^{\prime}\right) I_{2}\right\} X_{2}-(d+\delta) I_{1}-\eta I_{1}, \quad I_{1}(0) \geq 0, \\
\frac{d I_{2}}{d t} & =\eta I_{1}-(d+\delta) I_{2}-\alpha I_{2}, \quad I_{2}(0) \geq 0, \\
\frac{d R}{d t} & =\alpha I_{2}-\gamma R-d R, \quad R(0) \geq 0 .
\end{aligned}
$$




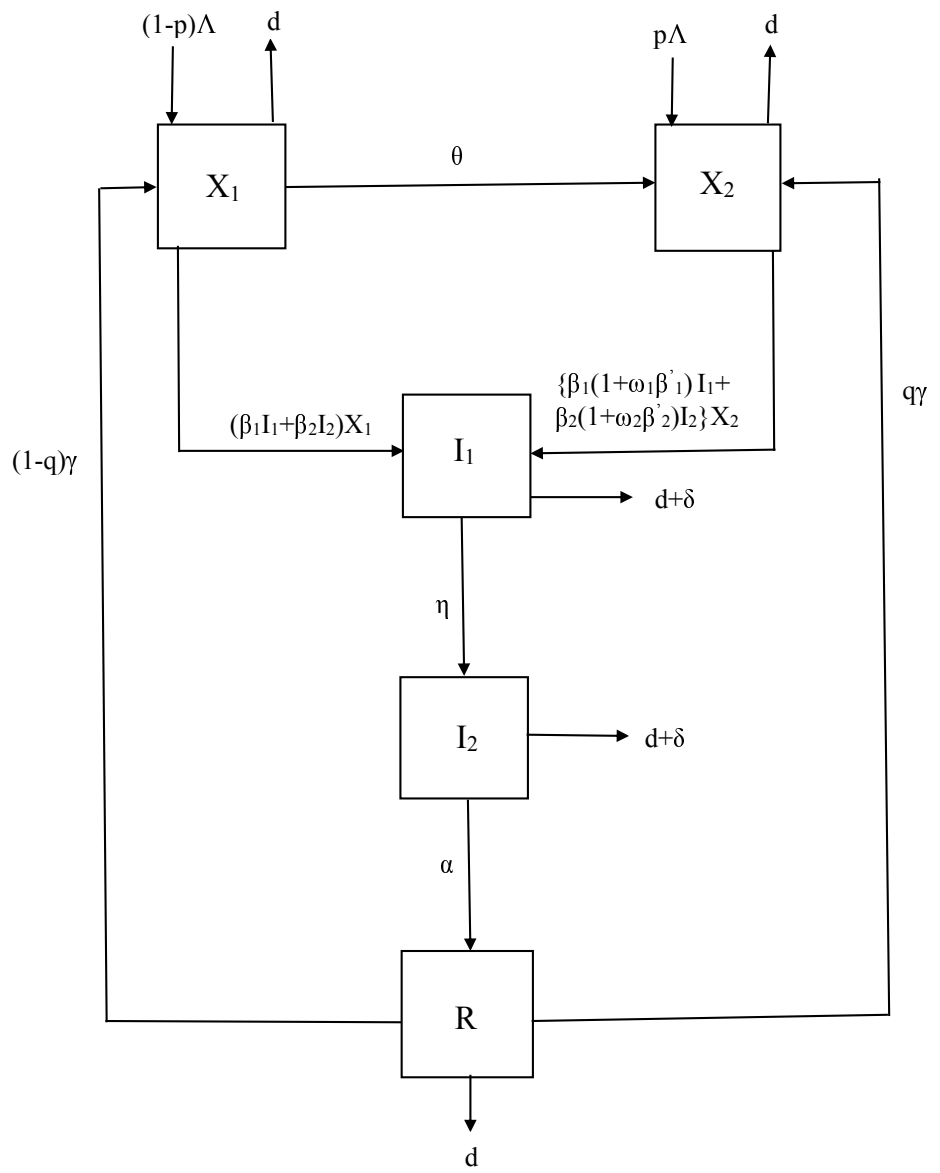

Fig. 1. Schematic diagram of system (1).

\section{POSITIVITY AND BOUNDEDNESS}

This section analyse the positivity and boundedness of the system variables to show the well-posedness of system (1).

The or e m 1. Solutions of system (1) starting in $\mathbb{R}_{+}^{5}$ are positive for all time.

P r o o f. Right hand side of system $(\overline{1})$ is continuous and locally Lipschitzian on $C$ (space of continuous functions) which implies that a unique solution $\left(X_{1}(t), X_{2}(t), I_{1}(t), I_{2}(t), R(t)\right)$ of (1) exists on $[0, \xi)$, where $0<\xi \leq+\infty$ [41]. We show that, $X_{1}(t)>0, \forall t \in[0, \xi)$. If it doesn't hold, then $\exists t_{1} \in[0, \xi)$ such that $X_{1}\left(t_{1}\right)=0, \dot{X}_{1}\left(t_{1}\right) \leq 0$ and $X_{1}(t)>0, \forall t \in\left[0, t_{1}\right)$. So there must have $I_{1}(t) \geq 0, \forall t \in\left[0, t_{1}\right)$. Suppose the statement is not true. Then $\exists t_{2} \in\left(0, t_{1}\right)$ such that $I_{1}\left(t_{2}\right)=0, \dot{I}_{1}\left(t_{2}\right)<0$ and $I_{1}(t) \geq 0, \forall t \in\left[0, t_{2}\right)$. Our claim is $I_{2}(t) \geq 0, \forall t \in\left[0, t_{2}\right)$. Again, if it is not true, then $\exists t_{3} \in\left(0, t_{2}\right)$ such that $I_{2}\left(t_{3}\right)=0, \dot{I}_{2}\left(t_{3}\right)<0$ and $I_{2}(t) \geq 0, \forall t \in\left[0, t_{3}\right)$. From $4^{\text {th }}$ equation of (1):

$$
\left.\frac{d I_{2}}{d t}\right|_{t=t_{3}}=\eta I_{1}\left(t_{3}\right)-(d+\delta+\alpha) I_{2}\left(t_{3}\right)=\eta I_{1}\left(t_{3}\right) \geq 0,
$$

which is a contradiction to $\dot{I}_{2}\left(t_{3}\right)<0$. So, $I_{2}(t) \geq 0, \forall t \in\left[0, t_{2}\right)$.

Next we claim $R(t) \geq 0, \forall t \in\left[0, t_{2}\right)$. Suppose it is not true. Then $\exists t_{4} \in\left(0, t_{2}\right)$ such that 
$R\left(t_{4}\right)=0, \dot{R}\left(t_{4}\right)<0$ and $R(t) \geq 0, \forall t \in\left[0, t_{4}\right)$. Now from the $5^{t h}$ equation of (1):

$$
\left.\frac{d R}{d t}\right|_{t=t_{4}}=\alpha I_{2}\left(t_{4}\right)-(d+\gamma) R\left(t_{4}\right)=\alpha I_{2}\left(t_{4}\right) \geq 0,
$$

which is a contradiction to $\dot{R}\left(t_{4}\right)<0$. So, $R(t) \geq 0, \forall t \in\left[0, t_{2}\right)$.

Next we claim $X_{2}(t)>0, \forall t \in\left[0, t_{2}\right)$. If it is not true, then $\exists t_{5} \in\left(0, t_{2}\right)$ such that $X_{2}\left(t_{5}\right)=$ $0, \dot{X}_{2}\left(t_{5}\right) \leq 0$ and $X_{2}(t)>0, \forall t \in\left[0, t_{5}\right)$. From the $2^{\text {nd }}$ equation of (1):

$$
\begin{aligned}
\left.\frac{d X_{2}}{d t}\right|_{t=t_{5}} & =p \Lambda+\theta X_{1}\left(t_{5}\right)-d X_{2}\left(t_{5}\right)+q \gamma R\left(t_{5}\right)-\left\{\beta_{1}\left(1+\omega_{1} \beta_{1}^{\prime}\right) I_{1}\left(t_{5}\right)+\beta_{2}\left(1+\omega_{2} \beta_{2}^{\prime}\right) I_{2}\left(t_{5}\right)\right\} X_{2}\left(t_{5}\right) \\
& =p \Lambda+\theta X_{1}\left(t_{5}\right)+q \gamma R\left(t_{5}\right)>0
\end{aligned}
$$

which is a contradiction to $\dot{X}_{2}\left(t_{5}\right) \leq 0$. So, $X_{2}(t)>0, \forall t \in\left[0, t_{2}\right)$.

Now from the $3^{\text {rd }}$ equation of (1):

$$
\begin{aligned}
\left.\frac{d I_{1}}{d t}\right|_{t=t_{2}} & =\left(\beta_{1} I_{1}\left(t_{2}\right)+\beta_{2} I_{2}\left(t_{2}\right)\right) X_{1}\left(t_{2}\right)+\left\{\beta_{1}\left(1+\omega_{1} \beta_{1}^{\prime}\right) I_{1}\left(t_{2}\right)+\beta_{2}\left(1+\omega_{2} \beta_{2}^{\prime}\right) I_{2}\left(t_{2}\right)\right\} X_{2}\left(t_{2}\right) \\
& -(d+\delta) I_{1}\left(t_{2}\right)-\eta I_{1}\left(t_{2}\right) \\
& =\beta_{2} I_{2}\left(t_{2}\right) X_{1}\left(t_{2}\right)+\beta_{2}\left(1+\omega_{2} \beta_{2}^{\prime}\right) I_{2}\left(t_{2}\right) X_{2}\left(t_{2}\right) \geq 0,
\end{aligned}
$$

which is a contradiction to $\dot{I}_{1}\left(t_{2}\right)<0$. So, $I_{1}(t) \geq 0, \forall t \in\left[0, t_{1}\right)$. Hence, $X_{2}(t)>0, I_{2}(t) \geq$ $0, R(t) \geq 0, \forall t \in\left[0, t_{1}\right)$.

Again from the $1^{\text {st }}$ equation of (1):

$$
\begin{aligned}
\left.\frac{d X_{1}}{d t}\right|_{t=t_{1}} & =(1-p) \Lambda-d X_{1}\left(t_{1}\right)-\theta X_{1}\left(t_{1}\right)+(1-q) \gamma R\left(t_{1}\right)-\left(\beta_{1} I_{1}\left(t_{1}\right)+\beta_{2} I_{2}\left(t_{1}\right)\right) X_{1}\left(t_{1}\right) \\
& =(1-p) \Lambda+(1-q) \gamma R\left(t_{1}\right)>0,
\end{aligned}
$$

which is a contradiction to $\dot{X}_{1}\left(t_{1}\right) \leq 0$. It shows that $X_{1}(t)>0, \forall t \in[0, \xi)$. By the previous steps we have $X_{2}>0, I_{1} \geq 0, I_{2} \geq 0$ and $R \geq 0 \forall t \in[0, \xi)$, where $0<\xi \leq+\infty$. Hence the theorem.

The o r e m 2. All solutions of system (1) starting in $\mathbb{R}_{+}^{5}$ are uniformly bounded.

Pro of.

$$
\text { Consider, } \begin{aligned}
N(t) & =X_{1}(t)+X_{2}(t)+I_{1}(t)+I_{2}(t)+R(t) . \\
\therefore \frac{d N}{d t} & =\Lambda-d N-\delta\left(I_{1}+I_{2}\right) \leq \Lambda-d N .
\end{aligned}
$$

The solution $N(t)$ has the following property:

$0<N(t) \leq N(0) e^{-d t}+\frac{\Lambda}{d}\left(1-e^{-d t}\right)$, where $N(0)=X_{1}(0)+X_{2}(0)+I_{1}(0)+I_{2}(0)+R(0)$.

As $t \rightarrow \infty, 0<N(t) \leq \frac{\Lambda}{d}$.

Therefore, all solutions of system (1) enter into the region:

$$
\Omega=\left\{\left(X_{1}, X_{2}, I_{1}, I_{2}, R\right) \in \mathbb{R}_{+}^{5}: 0<N(t) \leq \frac{\Lambda}{d}\right\}
$$




\section{EQUILIBRIUM ANALYSIS}

In this section, we analyse the equilibrium points of system (1) along with their feasibility conditions. For epidemiological models, usually basic reproduction number $R_{0}$ determines the existence of endemic equilibrium and stability of disease-free equilibrium of a system. System (1) has following equilibrium points:

(1) Disease-free equilibrium (DFE): $E_{0}\left(X_{10}, X_{20}, 0,0,0\right)=\left(\frac{(1-p) \Lambda}{d+\theta}, \frac{\Lambda(p d+\theta)}{d(d+\theta)}, 0,0,0\right)$. (2) Endemic equilibrium: $E^{*}\left(X_{1}^{*}, X_{2}^{*}, I_{1}^{*}, I_{2}^{*}, R^{*}\right)$.

\subsection{Basic reproduction number $\left(R_{0}\right)$}

Ability of an infectious disease to invade a population is one of the important concerns. Basic reproduction number $R_{0}$ is the number of average number of newly infective individuals generated by a single infectious individual when introduced into a susceptible population. Method developed by van den Driessche and Watmough [42] has been used here to determine $R_{0}$ of system (1).

For this system, infection is introduced only in asymptomatic phase and the infected compartments are asymptomatic $\left(I_{1}\right)$ and symptomatic stages $\left(I_{2}\right)$. Let us take $x \equiv\left(I_{1}, I_{2}\right)$. Third and fourth equations of system (1) can be written as:

$$
\begin{gathered}
\frac{d x}{d t}=\mathfrak{F}(x)-v(x), \\
\mathfrak{F}(x)=\left(\begin{array}{c}
\left(\beta_{1} I_{1}+\beta_{2} I_{2}\right) X_{1}+\left\{\beta_{1}\left(1+\omega_{1} \beta_{1}^{\prime}\right) I_{1}+\beta_{2}\left(1+\omega_{2} \beta_{2}^{\prime}\right) I_{2}\right\} X_{2} \\
0
\end{array}\right), \\
v(x)=\left(\begin{array}{c}
(d+\delta+\eta) I_{1} \\
-\eta I_{1}+(d+\delta+\alpha) I_{2}
\end{array}\right),
\end{gathered}
$$

where $\mathfrak{F}(x)$ has only new infection term and $v(x)$ has the other terms. Now the corresponding linearized matrices of $\mathfrak{F}(x)$ and $\vee(x)$ evaluated at disease-free equilibrium $E_{0}=$ $\left(X_{10}, X_{20}, 0,0,0\right)$ are respectively

$$
\begin{gathered}
F=(D \mathfrak{F}(x))\left(E_{0}\right)=\left(\begin{array}{cc}
\beta_{1} X_{10}+\beta_{1}\left(1+\omega_{1} \beta_{1}^{\prime}\right) X_{20} & \beta_{2} X_{10}+\beta_{2}\left(1+\omega_{2} \beta_{2}^{\prime}\right) X_{20} \\
0 & 0
\end{array}\right), \\
V=(D v(x))\left(E_{0}\right)=\left(\begin{array}{cc}
(d+\delta+\eta) & 0 \\
-\eta & (d+\delta+\alpha)
\end{array}\right) .
\end{gathered}
$$

Consider, $A=\beta_{1}(d+\delta+\alpha)+\beta_{2} \eta$ and $B=\beta_{1}\left(1+\omega_{1} \beta_{1}^{\prime}\right)(d+\delta+\alpha)+\beta_{2}\left(1+\omega_{2} \beta_{2}^{\prime}\right) \eta$. $R_{0}$ is the spectral radius of the next generation matrix $F V^{-1}$. So, the basic reproduction number 
of system (1) is [42]:

$$
\begin{aligned}
R_{0} & =\frac{\eta\left\{\beta_{2} X_{10}+\beta_{2}\left(1+\omega_{2} \beta_{2}^{\prime}\right) X_{20}\right\}+(d+\delta+\alpha)\left\{\beta_{1} X_{10}+\beta_{1}\left(1+\omega_{1} \beta_{1}^{\prime}\right) X_{20}\right\}}{(d+\delta+\eta)(d+\delta+\alpha)} \\
& =\frac{\left\{\beta_{1}(d+\delta+\alpha)+\beta_{2} \eta\right\} X_{10}+\left\{\beta_{1}\left(1+\omega_{1} \beta_{1}^{\prime}\right)(d+\delta+\alpha)+\beta_{2}\left(1+\omega_{2} \beta_{2}^{\prime}\right) \eta\right\} X_{20}}{(d+\delta+\eta)(d+\delta+\alpha)} \\
& =\frac{A X_{10}+B X_{20}}{(d+\delta+\eta)(d+\delta+\alpha)} \\
& =\frac{\Lambda\{A(1-p) d+B(p d+\theta)\}}{d(d+\theta)(d+\delta+\eta)(d+\delta+\alpha)} .
\end{aligned}
$$

Existence of endemic equilibrium point $E^{*}\left(X_{1}^{*}, X_{2}^{*}, I_{1}^{*}, I_{2}^{*}, R^{*}\right)$ :

Let, $A=\beta_{1}(d+\delta+\alpha)+\beta_{2} \eta, B=\beta_{1}\left(1+\omega_{1} \beta_{1}^{\prime}\right)(d+\delta+\alpha)+\beta_{2}\left(1+\omega_{2} \beta_{2}^{\prime}\right) \eta$ and $C=(d+\gamma)(d+\delta+\eta)(d+\delta+\alpha)$. From system (1), we have:

$$
\begin{array}{r}
(1-p) \Lambda-d X_{1}^{*}-\theta X_{1}^{*}+(1-q) \gamma R^{*}-\left(\beta_{1} I_{1}^{*}+\beta_{2} I_{2}^{*}\right) X_{1}^{*}=0, \\
p \Lambda+\theta X_{1}^{*}-d X_{2}^{*}+q \gamma R^{*}-\left\{\beta_{1}\left(1+\omega_{1} \beta_{1}^{\prime}\right) I_{1}^{*}+\beta_{2}\left(1+\omega_{2} \beta_{2}^{\prime}\right) I_{2}^{*}\right\} X_{2}^{*}=0 \\
\left(\beta_{1} I_{1}^{*}+\beta_{2} I_{2}^{*}\right) X_{1}^{*}+\left\{\beta_{1}\left(1+\omega_{1} \beta_{1}^{\prime}\right) I_{1}^{*}+\beta_{2}\left(1+\omega_{2} \beta_{2}^{\prime}\right) I_{2}^{*}\right\} X_{2}^{*}-(d+\delta) I_{1}^{*}-\eta I_{1}^{*}=0, \\
\eta I_{1}^{*}-(d+\delta) I_{2}^{*}-\alpha I_{2}^{*}=0, \\
\alpha I_{2}^{*}-(\gamma+d) R^{*}=0 .
\end{array}
$$

Solving:

$I_{1}^{*}=\left(\frac{d+\delta+\alpha}{\eta}\right) I_{2}^{*}, \quad R^{*}=\left(\frac{\alpha}{d+\gamma}\right) I_{2}^{*}, \quad X_{1}^{*}=\frac{\eta\left\{\Lambda(1-p)(d+\gamma)+\gamma \alpha(1-q) I_{2}^{*}\right\}}{(d+\gamma)\left[\eta(d+\theta)+A I_{2}^{*}\right]}$,

$X_{2}^{*}=\frac{p \eta \Lambda(d+\gamma)\left[\eta(d+\theta)+A I_{2}^{*}\right]+q \eta \gamma \alpha\left[\eta(d+\theta)+A I_{2}^{*}\right] I_{2}^{*}+\Lambda \theta \eta^{2}(1-p)(d+\gamma)+\theta \gamma \alpha \eta^{2}(1-q) I_{2}^{*}}{(d+\gamma)\left[\eta(d+\theta)+A I_{2}^{*}\right]\left[d \eta+B I_{2}^{*}\right]}$

and $I_{2}^{*}$ is a positive root of the equation

$$
P_{1} I_{2}^{2}+P_{2} I_{2}+P_{3}=0
$$

where, $P_{1}=A B[\gamma \eta \alpha(1-q)+q \eta \gamma \alpha-C]=$

$$
\begin{aligned}
& =-A B[d(d+\delta+\eta)(d+\delta+\alpha)+\gamma(d+\delta)(d+\delta+\alpha+\eta)]<0 ; \\
P_{2} & =A \eta\{d \gamma \alpha \eta(1-q)+B \Lambda(1-p)(d+\gamma)\}+B\left\{A p \eta \Lambda(d+\gamma)+\gamma \alpha \eta^{2}(q d+\theta)\right\}- \\
& -C \eta\{A d+B(d+\theta)\}, \\
P_{3} & =C d(d+\theta) \eta^{2}\left[\frac{\Lambda\{A(1-p) d+B(p d+\theta)}{d(d+\theta)(d+\delta+\eta)(d+\delta+\alpha)}-1\right]= \\
& =C d \eta^{2}(d+\theta)\left(R_{0}-1\right) .
\end{aligned}
$$

Here $P_{3}<0(>0)$ according as $R_{0}<1(>1)$. So, for $R_{0}>1$, we get a unique equilibrium point while for $R_{0}<1$, we get no feasible equilibrium point or at most two equilibrium points. Hence we have the following theorem.

Theorem 3. System (1) always has a disease-free equilibrium $E_{0}\left(X_{10}, X_{20}, 0,0,0\right)$ irrespective of any parametric values. Moreover, if $R_{0}>1$, it admits a unique endemic equilibrium $E^{*}\left(X_{1}^{*}, X_{2}^{*}, I_{1}^{*}, I_{2}^{*}, R^{*}\right)$. 


\section{SENSITIVITY ANALYSIS}

Basic reproduction number $R_{0}$ of system (1) depends on thirteen parameters, namely, recruitment rate $(\Lambda)$, probabilities of recruitment in susceptible and stressed population $(p, 1-$ $p)$, transmission rates $\left(\beta_{1}, \beta_{2}\right)$, stress levels $\left(\omega_{1}, \omega_{2}\right)$, levels upto which stress can be increased $\left(\beta_{1}^{\prime}, \beta_{2}^{\prime}\right)$, disease related death rate $(\delta)$, natural death rate $(d)$, progression rate of susceptible population into stressed class $(\theta)$, progression rate of asymptomatically infectious population into symptomatic class $(\eta)$, progression rate of symptomatically infectious population into recovered class $(\alpha)$. Now among all these parameters, we can not control some of the parameters like $\Lambda, p, d, \delta, \eta$.

Now, $R_{0}=\frac{\Lambda\left[d A+\theta B+p d\left\{\omega_{1} \beta_{1} \beta_{1}^{\prime}(d+\delta+\alpha)+\omega_{2} \beta_{2} \beta_{2}^{\prime} \eta\right\}\right]}{d(d+\theta)(d+\delta+\eta)(d+\delta+\alpha)}$, where $A=\beta_{1}(d+\delta+\alpha)+\beta_{2} \eta$ and $B=\beta_{1}\left(1+\omega_{1} \beta_{1}^{\prime}\right)(d+\delta+\alpha)+\beta_{2}\left(1+\omega_{2} \beta_{2}^{\prime}\right) \eta$ and from the expression of $R_{0}$ :

$$
\begin{aligned}
& \frac{\partial R_{0}}{\partial \beta_{1}}=\frac{\Lambda\left\{d+\theta\left(1+\omega_{1} \beta_{1}^{\prime}\right)+p d \omega_{1} \beta_{1}^{\prime}\right\}}{d(d+\theta)(d+\delta+\eta)}>0 \\
& \frac{\partial R_{0}}{\partial \beta_{2}}=\frac{\Lambda \eta\left[d+\theta\left(1+\omega_{2} \beta_{2}^{\prime}\right)+p d \omega_{2} \beta_{2}^{\prime}\right]}{d(d+\theta)(d+\delta+\eta)(d+\delta+\alpha)}>0 \\
& \frac{\partial R_{0}}{\partial \beta_{1}^{\prime}}=\frac{\Lambda \beta_{1} \omega_{1}(\theta+p d)}{d(d+\theta)(d+\delta+\eta)}>0 \\
& \frac{\partial R_{0}}{\partial \beta_{2}^{\prime}}=\frac{\Lambda \beta_{2} \omega_{2} \eta(\theta+p d)}{d(d+\theta)(d+\delta+\eta)(d+\delta+\alpha)}>0 \\
& \frac{\partial R_{0}}{\partial \omega_{1}}=\frac{\Lambda \beta_{1} \beta_{1}^{\prime}(\theta+p d)}{d(d+\theta)(d+\delta+\eta)}>0 \\
& \frac{\partial R_{0}}{\partial \omega_{2}}=\frac{\Lambda \beta_{2} \beta_{2}^{\prime} \eta(\theta+p d)}{d(d+\theta)(d+\delta+\eta)(d+\delta+\alpha)}>0 \\
& \frac{\partial R_{0}}{\partial \alpha}=-\frac{\Lambda \beta_{2} \eta\left[\theta\left(1+\omega_{2} \beta_{2}^{\prime}\right)+p d \omega_{2} \beta_{2}^{\prime}\right]}{d(d+\theta)(d+\delta+\eta)(d+\delta+\alpha)^{2}}<0 \\
& \frac{\partial R_{0}}{\partial \theta}=\frac{\Lambda d\left\{\beta_{1}\left(1+\omega_{1} \beta_{1}^{\prime}\right)(d+\delta+\alpha)+\beta_{2}\left(1+\omega_{2} \beta_{2}^{\prime}\right) \eta\right\}}{d(d+\theta)^{2}(d+\delta+\eta)(d+\delta+\alpha)}>0
\end{aligned}
$$

In order to examine the sensitivity of $R_{0}$ to the parameters $\beta_{1}, \beta_{2}, \omega_{1}, \omega_{2}, \beta_{1}^{\prime}, \beta_{2}^{\prime}, \alpha$ and $\theta$, we compute normalized forward sensitivity index with respect to each of these parameters using the method of Arriola and Hyman [43]:

$$
\begin{aligned}
& \Gamma_{\beta_{1}}=\left|\frac{\frac{\partial R_{0}}{R_{0}}}{\frac{\partial \beta_{1}}{\beta_{1}}}\right|=\left|\frac{\beta_{1}}{R_{0}} \frac{\partial R_{0}}{\partial \beta_{1}}\right|=\left|\frac{\beta_{1}(d+\delta+\alpha)\left\{d+\theta\left(1+\omega_{1} \beta_{1}^{\prime}\right)+p d \omega_{1} \beta_{1}^{\prime}\right\}}{d A+\theta B+p d\left\{\omega_{1} \beta_{1} \beta_{1}^{\prime}(d+\delta+\alpha)+\omega_{2} \beta_{2} \beta_{2}^{\prime} \eta\right\}}\right|, \\
& \Gamma_{\beta_{2}}=\left|\frac{\frac{\partial R_{0}}{R_{0}}}{\frac{\partial \beta_{2}}{\beta_{2}}}\right|=\left|\frac{\beta_{2}}{R_{0}} \frac{\partial R_{0}}{\partial \beta_{2}}\right|=\left|\frac{\beta_{2} \eta\left[d+\theta\left(1+\omega_{2} \beta_{2}^{\prime}\right)+p d \omega_{2} \beta_{2}^{\prime}\right]}{d A+\theta B+p d\left\{\omega_{1} \beta_{1} \beta_{1}^{\prime}(d+\delta+\alpha)+\omega_{2} \beta_{2} \beta_{2}^{\prime} \eta\right\}}\right| \text {, } \\
& \Gamma_{\beta_{1}^{\prime}}=\left|\frac{\frac{\partial R_{0}}{R_{0}}}{\frac{\partial \beta_{1}^{\prime}}{\beta_{1}^{\prime}}}\right|=\left|\frac{\beta_{1}^{\prime}}{R_{0}} \frac{\partial R_{0}}{\partial \beta_{1}^{\prime}}\right|=\left|\frac{\beta_{1} \beta_{1}^{\prime} \omega_{1}(p d+\theta)(d+\delta+\alpha)}{d A+\theta B+p d\left\{\omega_{1} \beta_{1} \beta_{1}^{\prime}(d+\delta+\alpha)+\omega_{2} \beta_{2} \beta_{2}^{\prime} \eta\right\}}\right| \text {, } \\
& \Gamma_{\beta_{2}^{\prime}}=\left|\frac{\frac{\partial R_{0}}{R_{0}}}{\frac{\partial \beta_{2}^{\prime}}{\beta_{2}^{\prime}}}\right|=\left|\frac{\beta_{2}^{\prime}}{R_{0}} \frac{\partial R_{0}}{\partial \beta_{2}^{\prime}}\right|=\left|\frac{\beta_{2} \beta_{2}^{\prime} \omega_{2} \eta(p d+\theta)}{d A+\theta B+p d\left\{\omega_{1} \beta_{1} \beta_{1}^{\prime}(d+\delta+\alpha)+\omega_{2} \beta_{2} \beta_{2}^{\prime} \eta\right\}}\right|,
\end{aligned}
$$




$$
\begin{gathered}
\Gamma_{\omega_{1}}=\left|\frac{\frac{\partial R_{0}}{R_{0}}}{\frac{\partial \omega_{1}}{\omega_{1}}}\right|=\left|\frac{\omega_{1}}{R_{0}} \frac{\partial R_{0}}{\partial \omega_{1}}\right|=\left|\frac{\beta_{1} \beta_{1}^{\prime} \omega_{1}(p d+\theta)(d+\delta+\alpha)}{d A+\theta B+p d\left\{\omega_{1} \beta_{1} \beta_{1}^{\prime}(d+\delta+\alpha)+\omega_{2} \beta_{2} \beta_{2}^{\prime} \eta\right\}}\right|, \\
\Gamma_{\omega_{2}}=\left|\frac{\frac{\partial R_{0}}{R_{0}}}{\frac{\partial \omega_{2}}{\omega_{2}}}\right|=\left|\frac{\omega_{2}}{R_{0}} \frac{\partial R_{0}}{\partial \omega_{2}}\right|=\left|\frac{\beta_{2} \beta_{2}^{\prime} \omega_{2} \eta(p d+\theta)}{d A+\theta B+p d\left\{\omega_{1} \beta_{1} \beta_{1}^{\prime}(d+\delta+\alpha)+\omega_{2} \beta_{2} \beta_{2}^{\prime} \eta\right\}}\right|, \\
\Gamma_{\alpha}=\left|\frac{\frac{\partial R_{0}}{R_{0}}}{\frac{\partial \alpha}{\alpha}}\right|=\left|\frac{\alpha}{R_{0}} \frac{\partial R_{0}}{\partial \alpha}\right|=\left|\frac{\alpha \beta_{2} \eta\left\{+(p d+\theta) \beta_{2}^{\prime} \omega_{2}\right\}}{(d+\delta+\alpha)\left[d A+\theta B+p d\left\{\omega_{1} \beta_{1} \beta_{1}^{\prime}(d+\delta+\alpha)+\omega_{2} \beta_{2} \beta_{2}^{\prime} \eta\right\}\right]}\right|, \\
\Gamma_{\theta}=\left|\frac{\frac{\partial R_{0}}{R_{0}}}{\frac{\partial \theta}{\theta}}\right|=\left|\frac{\theta}{R_{0}} \frac{\partial R_{0}}{\partial \theta}\right|=\left|\frac{d \theta\left\{\beta_{1}\left(1+\omega_{1} \beta_{1}^{\prime}\right)(d+\delta+\alpha)+\beta_{2}\left(1+\omega_{2} \beta_{2}^{\prime}\right) \eta\right\}}{(d+\theta)\left[d A+\theta B+p d\left\{\omega_{1} \beta_{1} \beta_{1}^{\prime}(d+\delta+\alpha)+\omega_{2} \beta_{2} \beta_{2}^{\prime} \eta\right\}\right]}\right| .
\end{gathered}
$$

From the mentioned discussion, it is observed that the normalized sensitivity index for stress level $\beta_{1}^{\prime}$ is same as $\omega_{1}$ implying that the basic reproduction number $R_{0}$ is equally sensitive for both the parameters $\omega_{1}$ and $\beta_{1}^{\prime}$. Moreover, $R_{0}$ is equally sensitive for both $\omega_{2}$ and $\beta_{2}^{\prime}$ also. Also, all these four parameters are directly proportional with $R_{0}$, i.e., the increase in these parameters cause a increase in $R_{0}$ and decrease cause a decrease in $R_{0}$. So, this analysis shows that if the stress levels increase, then disease can invade into population quickly. Also, the disease transmission coefficients $\beta_{1}, \beta_{2}$ are directly proportional with $R_{0}$ and it is quite obvious that increasing transmission rates cause quick invasion of disease in susceptible population. It is also evident that, more infection can spread in population if more people move into stressed class. The sensitivity index for $\theta$ proves that this parameter has a direct proportion with $R_{0}$. On the contrary, $\alpha$ maintains a inverse proportion with $R_{0}$, i.e., increment in the parameter leads to a decrease in $R_{0}$ and if this decreases, $R_{0}$ increases. From the calculations, it is obtained that $R_{0}$ is more sensitive to changes in $\beta_{1}^{\prime}, \beta_{2}^{\prime}$ or even $\omega_{1}, \omega_{2}$ than $\beta_{1}, \beta_{2}$ and $\theta$. So, it is sensible to focus on $\beta_{i}^{\prime}, \omega_{i}$ for $i=1,2$ which are stress levels and the extents upto which stresses can be increased.

\section{STABILITY ANALYSIS}

Now we are going to discuss the local stability criterion for the equilibrium points. The Jacobian matrix of system (1) is as follows:

$$
\bar{J}=\left(\begin{array}{ccccc}
a_{11} & a_{12} & a_{13} & a_{14} & a_{15} \\
a_{21} & a_{22} & a_{23} & a_{24} & a_{25} \\
a_{31} & a_{32} & a_{33} & a_{34} & a_{35} \\
a_{41} & a_{42} & a_{43} & a_{44} & a_{45} \\
a_{51} & a_{52} & a_{53} & a_{54} & a_{55}
\end{array}\right)
$$

where $a_{11}=-(d+\theta)-\left(\beta_{1} I_{1}+\beta_{2} I_{2}\right), a_{12}=0, a_{13}=-\beta_{1} X_{1}, a_{14}=\beta_{2} X_{1}, a_{15}=(1-q) \gamma$; $a_{21}=\theta, a_{22}=-d-\left\{\beta_{1}\left(1+\omega_{1} \beta_{1}^{\prime}\right) I_{1}+\beta_{2}\left(1+\omega_{2} \beta_{2}^{\prime}\right) I_{2}\right\}, a_{23}=-\beta_{1}\left(1+\omega_{1} \beta_{1}^{\prime}\right) X_{2}, a_{24}=$ $-\beta_{2}\left(1+\omega_{2} \beta_{2}^{\prime}\right) X_{2}, a_{25}=q \gamma$

$a_{31}=\beta_{1} I_{1}+\beta_{2} I_{2}, a_{32}=\beta_{1}\left(1+\omega_{1} \beta_{1}^{\prime}\right) I_{1}+\beta_{2}\left(1+\omega_{2} \beta_{2}^{\prime}\right) I_{2}, a_{33}=\beta_{1} X_{1}+\beta_{1}\left(1+\omega_{1} \beta_{1}^{\prime}\right) X_{2}-$ $(d+\delta+\eta), a_{34}=\beta_{2} X_{1}+\beta_{2}\left(1+\omega_{2} \beta_{2}^{\prime}\right) X_{2}, a_{35}=0$

$a_{41}=0, a_{42}=0, a_{43}=\eta, a_{44}=-(d+\delta+\alpha), a_{45}=0$

$a_{51}=0, a_{52}=0, a_{53}=0, a_{54}=\alpha, a_{55}=-(d+\gamma)$. 


\subsection{Local Stability of $E_{0}$}

Jacobian matrix corresponding to equilibrium point $E_{0}$ is given by:

$$
\left.\bar{J}\right|_{E_{0}}=\left(\begin{array}{ccccc}
-(d+\theta) & 0 & -\beta_{1} X_{10} & -\beta_{2} X_{10} & (1-q) \gamma \\
\theta & -d & -\beta_{1}\left(1+\omega_{1} \beta_{1}^{\prime}\right) X_{20} & -\beta_{2}\left(1+\omega_{2} \beta_{2}^{\prime}\right) X_{20} & q \gamma \\
0 & 0 & a_{33} & \beta_{2} X_{10}+\beta_{2}\left(1+\omega_{2} \beta_{2}^{\prime}\right) X_{20} & 0 \\
0 & 0 & \eta & -(d+\delta+\alpha) & 0 \\
0 & 0 & 0 & \alpha & -(d+\gamma)
\end{array}\right),
$$

where, $a_{33}=\beta_{1} X_{10}+\beta_{1}\left(1+\omega_{1} \beta_{1}^{\prime}\right) X_{20}-(d+\delta+\eta)$. Eigenvalues of the characteristic equation of $\left.\bar{J}\right|_{E_{0}}$ are $\lambda_{1}=-(d+\theta), \lambda_{2}=-d, \lambda_{3}=-(d+\gamma)$, and other two eigenvalues are the roots of the equation $\lambda^{2}+P_{1} \lambda+P_{2}=0$, where, $P_{1}=2(d+\delta)+\eta+\alpha-\beta_{1} X_{10}-\beta_{1}\left(1+\omega_{1} \beta_{1}^{\prime}\right) X_{20}$ and $P_{2}=-\left[(d+\delta+\alpha) a_{33}+\eta\left\{\beta_{2} X_{10}+\beta_{2}\left(1+\omega_{2} \beta_{2}^{\prime}\right) X_{20}\right\}\right]=-(d+\delta+\alpha)(d+\delta+\eta)\left(R_{0}-1\right)$. So, $P_{2}>0$ when $R_{0}<1$. Now, the characteristic equation has roots with negative real parts only when $P_{1}, P_{2}>0$. So, we have the following theorem.

Theorem 4. The disease free equilibrium $E_{0}$ is locally asymptotically stable (LAS) if $R_{0}<1$ along with $\beta_{1} X_{10}+\beta_{1}\left(1+\omega_{1} \beta_{1}^{\prime}\right) X_{20}<2(d+\delta)+\eta+\alpha$.

\subsection{Global Stability of $E_{0}$}

For global stability conditions of the disease free equilibrium point $E_{0}$, approach from [44] is used here. Let system (1) can be written as follows

$$
\begin{aligned}
\frac{d X}{d t} & =F(X, Z), \\
\frac{d Z}{d t} & =G(X, Z), \quad G(X, O)=O,
\end{aligned}
$$

where $X \in \mathbb{R}^{m}$ and $Z \in \mathbb{R}^{n}$ denote the number of uninfected and infected population respectively. So, by this notation, the disease free equilibrium point $E_{0}$ is denoted by $P_{0}=$ $\left(X_{0}, O\right)$. The following two conditions guarantee the global stability of DFE:

(A1) $X_{0}$ is globally asymptotically stable for $\frac{d X}{d t}=F(X, O)$.

$(A 2) G(X, Z)=B Z-\widehat{G}(X, Z)$, where $\widehat{G}(X, Z) \geq O$ for all $(X, Z) \in \Omega$ and $B=D_{Z} G\left(X_{0}, O\right)$ is a M-matrix (M-matrix: matrix with non-negative off-diagonal elements) and $\Omega$ is the region where the solutions of the model exist biologically. Then the following lemma [44] states the condition for global stability of the disease free equilibrium point $E_{0}$.

Lemma: The equilibrium point $P_{0}=\left(X_{0}, O\right)$ is globally asymptotically stable for $R_{0} \leq 1$ when the stated conditions $(A 1)$ and $(A 2)$ are satisfied.

The or e m 5. The disease-free equilibrium point $E_{0}$ of system (1) is GAS when $R_{0}<1$.

Pro of. For system (1), (A1) is as follows

$$
F(X, 0)=\left(\begin{array}{c}
(1-p) \Lambda-(\theta+d) X_{1} \\
p \Lambda+\theta X_{1}-d X_{2}
\end{array}\right) .
$$

The characteristic polynomial of the system is given by $(\lambda+d)(\lambda+(d+\theta))=0$ which gives the eigenvalues as $\lambda_{1}=-d$ and $\lambda_{2}=-(d+\theta)$. Hence $X=X_{0}$ is globally asymptotically 
stable. Now we have

$$
\begin{aligned}
& G(X, Z)=B Z-\widehat{G}(X, Z)= \\
& =\left(\begin{array}{ccc}
\beta_{1} X_{10}+\beta_{1}\left(1+\omega_{1} \beta_{1}^{\prime}\right) X_{20}-(d+\delta+\eta) & \beta_{2} X_{10}+\beta_{2}\left(1+\omega_{2} \beta_{2}^{\prime}\right) X_{20} & 0 \\
\eta & -(d+\delta+\alpha) & 0 \\
0 & \alpha & -(d+\gamma)
\end{array}\right)\left(\begin{array}{c}
I_{1} \\
I_{2} \\
R
\end{array}\right)- \\
& -\left(\begin{array}{c}
{\left[\left\{\beta_{1} X_{10}+\beta_{1}\left(1+\omega_{1} \beta_{1}^{\prime}\right) X_{20}\right\} I_{1}+\left\{\beta_{2} X_{10}+\beta_{2}\left(1+\omega_{2} \beta_{2}^{\prime}\right) X_{20}\right\} I_{2}\right.} \\
\left.-\left(\beta_{1} I_{1}+\beta_{2} I_{2}\right) X_{1}-\left\{\beta_{1}\left(1+\omega_{1} \beta_{1}^{\prime}\right) I_{1}+\beta_{2}\left(1+\omega_{2} \beta_{2}^{\prime}\right) I_{2}\right\} X_{2}\right] \\
0 \\
0
\end{array}\right)
\end{aligned}
$$

Here, $B$ is a M-matrix and $\widehat{G}(X, Z) \geq O$. Hence, the conditions stated in $(A 1)$ and $(A 2)$ are satisfied and the theorem follows.

\subsection{Local Stability of $E^{*}$}

Th e or e m 6. When $R_{0}>1$, the endemic equilibrium point $E^{*}$ of system (1) is LAS under the conditions (i), (ii), (iii) and (iv) (stated in the proof).

Pr o o f. From (4), the Jacobian matrix corresponding to $E^{*}$ is

$$
\bar{J}=\left(\begin{array}{ccccc}
a_{11} & 0 & a_{13} & a_{14} & a_{15} \\
a_{21} & a_{22} & a_{23} & a_{24} & a_{25} \\
a_{31} & a_{32} & a_{33} & a_{34} & 0 \\
0 & 0 & a_{43} & a_{44} & 0 \\
0 & 0 & 0 & a_{54} & a_{55}
\end{array}\right)
$$

where $a_{11}=-(d+\theta)-\left(\beta_{1} I_{1}^{*}+\beta_{2} I_{2}^{*}\right), a_{13}=-\beta_{1} X_{1}^{*}, a_{14}=\beta_{2} X_{1}^{*}, a_{15}=(1-q) \gamma$;

$a_{21}=\theta, a_{22}=-d-\left\{\beta_{1}\left(1+\omega_{1} \beta_{1}^{\prime}\right) I_{1}^{*}+\beta_{2}\left(1+\omega_{2} \beta_{2}^{\prime}\right) I_{2}^{*}\right\}, a_{23}=-\beta_{1}\left(1+\omega_{1} \beta_{1}^{\prime}\right) X_{2}^{*}, a_{24}=$ $-\beta_{2}\left(1+\omega_{2} \beta_{2}^{\prime}\right) X_{2}^{*}, a_{25}=q \gamma$

$a_{31}=\beta_{1} I_{1}^{*}+\beta_{2} I_{2}^{*}, a_{32}=\beta_{1}\left(1+\omega_{1} \beta_{1}^{\prime}\right) I_{1}^{*}+\beta_{2}\left(1+\omega_{2} \beta_{2}^{\prime}\right) I_{2}^{*}, a_{33}=\beta_{1} X_{1}^{*}+\beta_{1}(1+$ $\left.\omega_{1} \beta_{1}^{\prime}\right) X_{2}^{*}-(d+\delta+\eta), a_{34}=\beta_{2} X_{1}^{*}+\beta_{2}\left(1+\omega_{2} \beta_{2}^{\prime}\right) X_{2}^{*}$

$a_{43}=\eta, a_{44}=-(d+\delta+\alpha) ; a_{54}=\alpha, a_{55}=-(d+\gamma)$.

Characteristic equation of $\left.\bar{J}\right|_{E_{1}}$ is $\lambda^{5}+Q_{1} \lambda^{4}+Q_{2} \lambda^{3}+Q_{3} \lambda^{2}+Q_{4} \lambda+Q_{5}=0$,

where $Q_{1}=-\left(a_{11}+a_{22}+a_{33}+a_{44}+a_{55}\right)$,

$$
\begin{aligned}
Q_{2} & =a_{55}\left(a_{11}+a_{22}+a_{33}+a_{44}\right)+a_{33} a_{44}+a_{11} a_{22}+\left(a_{11}+a_{22}\right)\left(a_{33}+a_{44}\right) \\
& -a_{34} a_{43} a_{23} a_{32}-a_{13} a_{31} \\
Q_{3} & =-a_{55}\left\{a_{33} a_{44}+a_{11} a_{22}+\left(a_{11}+a_{22}\right)\left(a_{33}+a_{44}\right)\right\}-a_{33} a_{44}\left(a_{11}+a_{22}\right) \\
& -a_{11} a_{22}\left(a_{33}+a_{44}\right)+a_{34} a_{43}\left(a_{11}+a_{22}+a_{55}\right)+a_{23} a_{32}\left(a_{11}+a_{44}+a_{55}\right) \\
& -a_{43} a_{32} a_{24}-a_{21} a_{13} a_{32}+a_{13} a_{31}\left(a_{22}+a_{44}+a_{55}\right)-a_{14} a_{43} a_{31} \\
Q_{4} & =a_{55}\left\{a_{33} a_{44}\left(a_{11}+a_{22}\right)+a_{11} a_{22}\left(a_{33}+a_{44}\right)\right\}+a_{11} a_{22} a_{33} a_{44} \\
& -a_{34} a_{43}\left\{a_{55}\left(a_{11}+a_{22}\right)+a_{11} a_{22}\right\}-a_{23} a_{32}\left\{a_{55}\left(a_{11}+a_{44}\right)+a_{11} a_{44}\right\} \\
& +a_{43} a_{32} a_{24}\left(a_{11}+a_{55}\right)+a_{21} a_{32} a_{13}\left(a_{44}+a_{55}\right)-a_{13} a_{31}\left\{a_{55}\left(a_{22}+a_{44}\right)+a_{22} a_{44}\right\} \\
& -a_{14} a_{43} a_{32} a_{21}+a_{14} a_{43} a_{31}\left(a_{22}+a_{55}\right)-a_{54} a_{43} a_{32} a_{25}-a_{54} a_{43} a_{31} a_{15}
\end{aligned}
$$




$$
\begin{aligned}
Q_{5} & =-a_{11} a_{22} a_{33} a_{44} a_{55}+a_{11} a_{22} a_{34} a_{43} a_{55}+a_{11} a_{23} a_{32} a_{44} a_{55} \\
& -a_{11} a_{55} a_{43} a_{32} a_{24}-a_{21} a_{13} a_{32} a_{44} a_{55}+a_{13} a_{31} a_{22} a_{44} a_{55}+a_{14} a_{43} a_{32} a_{21} a_{55} \\
& -a_{14} a_{43} a_{31} a_{22} a_{55}+a_{11} a_{54} a_{43} a_{32} a_{25}-a_{54} a_{43} a_{32} a_{21} a_{15}+a_{54} a_{43} a_{31} a_{15} a_{22} .
\end{aligned}
$$

So, by Routh-Hurwitz criterion, $E^{*}$ is locally asymptotically stable (LAS) if the following conditions hold: (i) $Q_{i}>0$ for $i=1,2,3,4,5$;

(ii) $Q_{3}\left(Q_{1} Q_{2}-Q_{3}\right)>0$

(iii) $\left(Q_{1} Q_{2}-Q_{3}\right) Q_{3}>Q_{1}^{2} Q_{4}-Q_{1} Q_{5}$,

(iv) $Q_{4}\left\{Q_{1}\left(Q_{2} Q_{3}-Q_{1} Q_{4}\right)-Q_{3}^{2}+Q_{1} Q_{5}\right\}>Q_{5}\left\{Q_{1}\left(Q_{2}^{2}-Q_{4}\right)-Q_{2} Q_{3}+Q_{5}\right\}$.

\subsection{Global Stability of $E^{*}$}

We check the global stability of the endemic equilibrium point $E^{*}$ of system (1) here.

Theorem 7. Assume that $R_{0}>1$ and consider

$$
\begin{aligned}
& P=\left(\beta_{2} X_{1}^{*} I_{2}^{*}+\beta_{2}\left(1+\omega_{2} \beta_{2}^{\prime}\right) X_{2}^{*} I_{2}^{*}-\eta I_{1}^{*}\right), \\
& Q=\left(\beta_{1} X_{1}^{*} I_{1}^{*}+\beta_{1}\left(1+\omega_{1} \beta_{1}^{\prime}\right) X_{2}^{*} I_{1}^{*}-\alpha I_{2}^{*}\right), \\
& U=\left(\Lambda+(d+\theta) X_{1}^{*}+d X_{2}^{*}+(d+\delta+\eta) I_{1}^{*}+(d+\delta+\alpha) I_{2}^{*}+(d+\gamma) R^{*}\right) .
\end{aligned}
$$

Then the endemic equilibrium $E^{*}$ of system (1) is globally asymptotically stable (GAS) in the region $\Xi=\left\{\left(X_{1}, X_{2}, I_{1}, I_{2}, R\right) \in \mathbb{R}_{+}^{5}: P I_{2}^{*} I_{1}+Q I_{1}^{*} I_{2}>U I_{1}^{*} I_{2}^{*}\right\}$.

Pro of. Consider a function $V$ as:

$$
\begin{aligned}
V(t) & =\left(X_{1}-X_{1}^{*}-X_{1}^{*} \ln \frac{X_{1}}{X_{1}^{*}}\right)+\left(X_{2}-X_{2}^{*}-X_{2}^{*} \ln \frac{X_{2}}{X_{2}^{*}}\right)+\left(I_{1}-I_{1}^{*}-I_{1}^{*} \ln \frac{I_{1}}{I_{1}^{*}}\right) \\
& +\left(I_{2}-I_{2}^{*}-I_{2}^{*} \ln \frac{I_{2}}{I_{2}^{*}}\right)+\left(R-R^{*}-R^{*} \ln \frac{R}{R^{*}}\right)
\end{aligned}
$$

Time derivative of $V$ computed along the solutions of system (1) is given by

$$
\begin{aligned}
& \frac{d V}{d t}=\left(1-\frac{X_{1}^{*}}{X_{1}}\right) \frac{d X_{1}}{d t}+\left(1-\frac{X_{2}^{*}}{X_{2}}\right) \frac{d X_{2}}{d t}+\left(1-\frac{I_{1}^{*}}{I_{1}}\right) \frac{d I_{1}}{d t}+\left(1-\frac{I_{2}^{*}}{I_{2}}\right) \frac{d I_{2}}{d t}+ \\
& +\left(1-\frac{R^{*}}{R}\right) \frac{d R}{d t}= \\
& =\left(1-\frac{X_{1}^{*}}{X_{1}}\right)\left[(1-p) \Lambda-d X_{1}-\theta X_{1}+(1-q) \gamma R-\left(\beta_{1} I_{1}+\beta_{2} I_{2}\right) X_{1}\right]+ \\
& +\left(1-\frac{X_{2}^{*}}{X_{2}}\right)\left[p \Lambda+\theta X_{1}-d X_{2}+q \gamma R-\left\{\beta_{1}\left(1+\omega_{1} \beta_{1}^{\prime}\right) I_{1}+\beta_{2}\left(1+\omega_{2} \beta_{2}^{\prime}\right) I_{2}\right\} X_{2}\right]+ \\
& +\left(1-\frac{I_{1}^{*}}{I_{1}}\right)\left[\left(\beta_{1} I_{1}+\beta_{2} I_{2}\right) X_{1}+\left\{\beta_{1}\left(1+\omega_{1} \beta_{1}^{\prime}\right) I_{1}+\beta_{2}\left(1+\omega_{2} \beta_{2}^{\prime}\right) I_{2}\right\} X_{2}-(d+\delta+\eta) I_{1}\right]+ \\
& +\left(1-\frac{I_{2}^{*}}{I_{2}}\right)\left[\eta I_{1}-(d+\delta+\alpha) I_{2}\right]+\left(1-\frac{R^{*}}{R}\right)\left[\alpha I_{2}-(\gamma+d) R\right] .
\end{aligned}
$$$$
\text { Let, } \frac{X_{1}}{X_{1}^{*}}=u, \quad \frac{X_{2}}{X_{2}^{*}}=v, \quad \frac{I_{1}}{I_{1}^{*}}=r, \quad \frac{I_{2}}{I_{2}^{*}}=s, \quad \frac{R}{R^{*}}=y \text {. }
$$ 


$$
\begin{aligned}
& \frac{d V}{d t}=(d+\theta) X_{1}^{*}\left(2-u-\frac{1}{u}\right)-(1-q) \gamma R^{*}\left(1-y-\frac{1}{u}+\frac{y}{u}\right)+\beta_{1} X_{1}^{*} I_{1}^{*}\left(1-u r-\frac{1}{u}+r\right)+ \\
& +\beta_{2} X_{1}^{*} I_{2}^{*}\left(1-u s+s-\frac{1}{u}\right)-\theta X_{1}^{*}\left(1-\frac{1}{v}-u+\frac{u}{v}\right)+d X_{2}^{*}\left(2-v-\frac{1}{v}\right)- \\
& -q \gamma R^{*}\left(1-y-\frac{1}{v}+\frac{y}{v}\right)+\beta_{1}\left(1+\omega_{1} \beta_{1}^{\prime}\right) X_{2}^{*} I_{1}^{*}\left(1-v r-\frac{1}{v}+r\right)+\beta_{2}\left(1+\omega_{2} \beta_{2}^{\prime}\right) X_{2}^{*} I_{2}^{*} \times \\
& \times\left(1-v s-\frac{1}{v}+s\right)-\beta_{1} X_{1}^{*} I_{1}^{*}\left(1-u r-\frac{1}{r}+u\right)-\beta_{2} X_{1}^{*} I_{2}^{*}\left(1-u s-\frac{1}{r}+\frac{u s}{r}\right)- \\
& -\beta_{1}\left(1+\omega_{1} \beta_{1}^{\prime}\right) X_{2}^{*} I_{1}^{*}\left(1-v r-\frac{1}{r}+v\right) \beta_{2}\left(1+\omega_{2} \beta_{2}^{\prime}\right) X_{2}^{*} I_{2}^{*}\left(1-v s-\frac{1}{r}+\frac{v s}{r}\right)+ \\
& +(d+\delta+\eta) I_{1}^{*}\left(2-r-\frac{1}{r}\right)-\eta I_{1}^{*}\left(1-r-\frac{1}{s}+\frac{r}{s}\right)+(d+\delta+\alpha) I_{2}^{*}\left(2-s-\frac{1}{s}\right)- \\
& -\alpha I_{2}^{*}\left(1-s-\frac{1}{y}+\frac{s}{t}\right)+(d+\gamma) R^{*}\left(2-y-\frac{1}{y}\right) .
\end{aligned}
$$

Steady state of system (1) at $E^{*}$ gives

$$
\begin{array}{r}
(1-p) \Lambda-d X_{1}^{*}-\theta X_{1}^{*}+(1-q) \gamma R^{*}-\left(\beta_{1} I_{1}^{*}+\beta_{2} I_{2}^{*}\right) X_{1}^{*}=0 \\
p \Lambda+\theta X_{1}^{*}-d X_{2}^{*}+q \gamma R^{*}-\left\{\beta_{1}\left(1+\omega_{1} \beta_{1}^{\prime}\right) I_{1}^{*}+\beta_{2}\left(1+\omega_{2} \beta_{2}^{\prime}\right) I_{2}^{*}\right\} X_{2}^{*}=0 \\
\left(\beta_{1} I_{1}^{*}+\beta_{2} I_{2}^{*}\right) X_{1}^{*}+\left\{\beta_{1}\left(1+\omega_{1} \beta_{1}^{\prime}\right) I_{1}^{*}+\beta_{2}\left(1+\omega_{2} \beta_{2}^{\prime}\right) I_{2}^{*}\right\} X_{2}^{*}-(d+\delta) I_{1}^{*}-\eta I_{1}^{*}=0 \\
\eta I_{1}^{*}-(d+\delta) I_{2}^{*}-\alpha I_{2}^{*}=0 \\
\alpha I_{2}^{*}-(\gamma+d) R^{*}=0,
\end{array}
$$

which leads to

$$
\begin{aligned}
\frac{d V}{d t} & =-X_{1}^{*}\left(d+\beta_{1} I_{1}^{*}\right) u-\frac{(1-p) \Lambda}{u}-d R^{*} y-\frac{p \Lambda}{v}-\left\{\beta_{1} X_{1}^{*} I_{1}^{*}+\beta_{1}\left(1+\omega_{1} \beta_{1}^{\prime}\right) X_{2}^{*} I_{1}^{*}-\alpha I_{2}^{*}\right\} s- \\
& -\left\{\beta_{2} X_{1}^{*} I_{2}^{*}+\beta_{2}\left(1+\omega_{2} \beta_{2}^{\prime}\right) X_{2}^{*} I_{2}^{*}-\eta I_{1}^{*}\right\} r-\left\{d+\beta_{1}\left(1+\omega_{1} \beta_{1}^{\prime}\right) I_{1}^{*}\right\} X_{2}^{*} v+\left\{\Lambda+(d+\theta) X_{1}^{*}+\right. \\
& \left.+d X_{2}^{*}+(d+\delta+\eta) I_{1}^{*}+(d+\delta+\alpha) I_{2}^{*}+(d+\gamma) R^{*}\right\} \leq \\
& \leq\left\{\Lambda+(d+\theta) X_{1}^{*}+d X_{2}^{*}+(d+\delta+\eta) I_{1}^{*}+(d+\delta+\alpha) I_{2}^{*}+(d+\gamma) R^{*}\right\}- \\
& -\frac{I_{1}}{I_{1}^{*}}\left\{\beta_{2} X_{1}^{*} I_{2}^{*}+\beta_{2}\left(1+\omega_{2} \beta_{2}^{\prime}\right) X_{2}^{*} I_{2}^{*}-\eta I_{1}^{*}\right\}-\frac{I_{2}}{I_{2}^{*}}\left\{\beta_{1} X_{1}^{*} I_{1}^{*}+\beta_{1}\left(1+\omega_{1} \beta_{1}^{\prime}\right) X_{2}^{*} I_{1}^{*}-\alpha I_{2}^{*}\right\}= \\
& =U-P \frac{I_{1}}{I_{1}^{*}}-Q \frac{I_{2}}{I_{2}^{*}}<0 .
\end{aligned}
$$

where, $P=\beta_{2} X_{1}^{*} I_{2}^{*}+\beta_{2}\left(1+\omega_{2} \beta_{2}^{\prime}\right) X_{2}^{*} I_{2}^{*}-\eta I_{1}^{*}, Q=\beta_{1} X_{1}^{*} I_{1}^{*}+\beta_{1}\left(1+\omega_{1} \beta_{1}^{\prime}\right) X_{2}^{*} I_{1}^{*}-\alpha I_{2}^{*}$ and $U=\Lambda+(d+\theta) X_{1}^{*}+d X_{2}^{*}+(d+\delta+\eta) I_{1}^{*}+(d+\delta+\alpha) I_{2}^{*}+(d+\gamma) R^{*}$. Therefore, $\frac{d V}{d t}<0$ in $\Xi$ and $\left.\frac{d V}{d t}\right|_{E^{*}}=0$. Hence, by Lyapunov LaSalle's theorem [45], $E^{*}$ is globally asymptotically stable in the interior of $\Xi$ for $R_{0}>1$.

\section{DIRECTION OF BIFURCATION AT $R_{0}=1$}

In system (1), an unique endemic equilibrium point $E^{*}$ exists only when $R_{0}>1$. When $R_{0}$ lies below unity, either there will be no endemic equilibrium point or at most two endemic equilibrium points. So, we have to search for the existence of a bifurcation around the equilibrium point when $R_{0}=1$. For this purpose let us state the central manifold theorem [46]:

Th e o r e m 8. Consider the following system of ODEs with a parameter $\Phi$ : 


$$
\frac{d x}{d t}=f(x, \Phi), f: \mathbb{R}^{n} \times \mathbb{R} \rightarrow \mathbb{R}^{n} \text { and } f \in C^{2}\left(\mathbb{R}^{n} \times \mathbb{R}\right),
$$

with $O_{e q}$ as an equilibrium of this system and $f\left(O_{e q}, \Phi\right)=O$ for all $\Phi$. Assume (a) $M=D_{x} f\left(O_{e q}, 0\right)=\left(\frac{\partial f_{i}}{\partial x_{j}}\left(O_{e q}, 0\right)\right)$ is the linearized matrix of the above system around the equilibrium point $O_{e q}$ with $\Phi$ evaluated at 0 . Zero is a simple eigenvalue of the matrix $M$ and all other eigenvalues of the matrix have negative real parts.

(b) Matrix $M$ has a non-negative right eigenvector $w$ and a left eigenvector $v$ corresponding to the zero eigenvalue.

Let $f_{k}$ be the $k^{\text {th }}$ component of $f$ and

$$
\begin{gathered}
a=\sum_{k, i, j=1}^{n} v_{k} w_{i} w_{j} \frac{\partial^{2} f_{k}}{\partial x_{i} \partial x_{j}}\left(O_{e q}, 0\right), \\
b=\sum_{k, i=1}^{n} v_{k} w_{i} \frac{\partial^{2} f_{k}}{\partial x_{i} \partial \Phi}\left(O_{e q}, 0\right),
\end{gathered}
$$

then the local dynamics of the system around $O_{e q}$ is totally determined by $a$ and $b$ as follows:

1. $a>0, b>0$. When $\Phi<0$ with $|\Phi| \ll 1, O_{e q}$ is locally asymptotically stable, and there exists a positive unstable equilibrium; when $0<\Phi \ll 1, O_{e q}$ is unstable and there exists a negative and locally asymptotically stable equilibrium.

2. $a<0, b<0$. When $\Phi<0$ with $|\Phi| \ll 1, O_{\text {eq }}$ is unstable; when $0<\Phi \ll 1, O_{\text {eq }}$ is locally asymptotically stable, and there exists a positive unstable equilibrium.

3. $a>0, b<0$. When $\Phi<0$ with $|\Phi| \ll 1, O_{e q}$ is unstable, and there exists a locally asymptotically stable negative equilibrium; when $0<\Phi \ll 1, O_{\text {eq }}$ is stable, and a positive unstable equilibrium appears.

4. $a<0, b>0$. When $\Phi$ changes from negative to positive, $O_{e q}$ changes its stability from stable to unstable. Correspondingly a negative unstable equilibrium becomes positive and locally asymptotically stable.

The non-negativity of components of the eigenvector $w$ is not necessary when corresponding component of equilibrium is positive. It is described in Remark 1 in [46].

The requirement that $w$ is non-negative in the previous theorem is not necessary and even when some components in $w$ are negative, we still can apply the theorem. But in this case, one has to compare the components of $w$ with the equilibrium because the general parameterization of the center manifold before the coordinate change is

$$
W^{c}=\left\{x_{0}+c(t) y+k(c, \Phi): v \cdot k(c, \Phi),|c| \leq c_{0}, c(0)=0\right\}
$$

given that $x_{0}$ is a non-negative equilibrium point of system (usually $x_{0}$ is the DFE). Hence, $x_{0}-2 \frac{b \Phi}{a}>0$ needs that $w(j)>0$ when $x_{0}(j)=0 . x_{0}(j)>0$ does not imply that $w(j)$ is positive.

Define $X_{1}=x_{1}, X_{2}=x_{2}, I_{1}=x_{3}, I_{2}=x_{4}$ and $R=x_{5}$, then system (1) can be rewritten 
as:

$$
\begin{aligned}
& \frac{d x_{1}}{d t}=(1-p) \Lambda-d x_{1}-\theta x_{1}+(1-q) \gamma x_{5}-\left(\beta_{1} x_{3}+\beta_{2} x_{4}\right) x_{1} \equiv f_{1}, \\
& \frac{d x_{2}}{d t}=p \Lambda+\theta x_{1}-d x_{2}+q \gamma x_{5}-\left\{\beta_{1}\left(1+\omega_{1} \beta_{1}^{\prime}\right) x_{3}+\beta_{2}\left(1+\omega_{2} \beta_{2}^{\prime}\right) x_{4}\right\} X_{2} \equiv f_{2}, \\
& \frac{d x_{3}}{d t}=\left(\beta_{1} x_{3}+\beta_{2} x_{4}\right) x_{1}+\left\{\beta_{1}\left(1+\omega_{1} \beta_{1}^{\prime}\right) x_{3}+\beta_{2}\left(1+\omega_{2} \beta_{2}^{\prime}\right) x_{4}\right\} x_{2}-(d+\delta) x_{3}-\eta x_{3} \equiv f_{3}, \\
& \frac{d x_{4}}{d t}=\eta x_{3}-(d+\delta) x_{4}-\alpha x_{4} \equiv f_{4} \\
& \frac{d x_{5}}{d t}=\alpha x_{4}-(\gamma+d) x_{5} \equiv f_{5}
\end{aligned}
$$

Let us consider $\Phi=\beta_{1}$ as bifurcation parameter for $R_{0}=1$. Thus at $\Phi=\Phi^{*}=$ $\beta_{1}^{*}, R_{0}=1$ gives $\beta_{1}^{*}=\frac{(d+\delta+\eta)(d+\delta+\alpha)-\beta_{2} \eta\left\{X_{10}+\left(1+\omega_{1} \beta_{1}^{\prime}\right) X_{20}\right\}}{(d+\delta+\alpha)\left\{X_{10}+\left(1+\omega_{1} \beta_{1}^{\prime}\right) X_{20}\right\}}$. The linearized matrix of system (5) at $E_{0}\left(X_{10}\left(\beta_{1}^{*}\right), X_{20}\left(\beta_{1}^{*}\right), 0,0,0\right) \equiv\left(X_{10}^{*}, X_{20}^{*}, 0,0,0\right)$ with bifurcation parameter $\beta_{1}=\beta_{1}^{*}$ is given by

$$
\left.\bar{J}\right|_{E_{0}}=\left(\begin{array}{ccccc}
-(d+\theta) & 0 & -\beta_{1}^{*} X_{10}^{*} & -\beta_{2} X_{10}^{*} & (1-q) \gamma \\
\theta & -d & -\beta_{1}^{*}\left(1+\omega_{1} \beta_{1}^{\prime}\right) X_{20}^{*} & -\beta_{2}\left(1+\omega_{2} \beta_{2}^{\prime}\right) X_{20}^{*} & q \gamma \\
0 & 0 & a_{33} & \beta_{2} X_{10}^{*}+\beta_{2}\left(1+\omega_{2} \beta_{2}^{\prime}\right) X_{20}^{*} & 0 \\
0 & 0 & \eta & -(d+\delta+\alpha) & 0 \\
0 & 0 & 0 & \alpha & -(d+\gamma)
\end{array}\right),
$$

where, $a_{33}=\beta_{1}^{*} X_{10}^{*}+\beta_{1}^{*}\left(1+\omega_{1} \beta_{1}^{\prime}\right) X_{20}^{*}-(d+\delta+\eta)$. Here, $\lambda_{1}=-(d+\theta), \lambda_{2}=-d, \lambda_{3}=$ $-(d+\gamma)$, and other two eigenvalues are the roots of the equation: $\lambda^{2}+P_{1} \lambda+P_{2}=0$, where, $P_{1}=2(d+\delta)+\eta+\alpha-\beta_{1}^{*} X_{10}^{*}-\beta_{1}^{*}\left(1+\omega_{1} \beta_{1}^{\prime}\right) X_{20}^{*}$ and $P_{2}=-(d+\delta+\alpha)(d+\delta+\eta)\left(R_{0}-1\right)$.

At $R_{0}=1, P_{2}=0$ resulting in one zero eigenvalue of $\left.\bar{J}\right|_{E_{0}}\left(\beta_{1}^{*}\right)$ and one eigenvalue with negative real part (by LAS condition). Right eigenvector corresponding to the zero eigenvalue of the matrix $\left.\bar{J}\right|_{E_{0}}\left(\beta_{1}^{*}\right)$ is denoted by $w=\left(w_{1}, w_{2}, w_{3}, w_{4}, w_{5}\right)^{T}$ where,

$$
\begin{aligned}
w_{1} & =d\left\{(1-q) \gamma \alpha \eta-\beta_{1}^{*} X_{10}^{*}(d+\delta+\alpha)(d+\gamma)-\beta_{2} X_{10}^{*} \eta(d+\gamma)\right\}, \\
w_{2} & =\left[\gamma \alpha \eta(\theta+q d)-\beta_{1}^{*}(d+\delta+\alpha)(d+\gamma)\left\{\theta X_{10}^{*}+\left(1+\omega_{1} \beta_{1}^{\prime}\right) X_{20}^{*}(d+\theta)\right\}\right. \\
& \left.-\beta_{2}\left\{X_{10}^{*} \eta \theta(d+\gamma)+\eta(d+\gamma)(d+\theta)\left(1+\omega_{2} \beta_{2}^{\prime}\right) X_{20}^{*}\right\}\right], \\
w_{3} & =d(d+\gamma)(d+\theta)(d+\delta+\alpha), \\
w_{4} & =d \eta(d+\gamma)(d+\theta), \\
w_{5} & =d \alpha \eta(d+\theta) .
\end{aligned}
$$

Also the left eigenvector of $\left.\bar{J}\right|_{E_{0}}\left(\beta_{1}^{*}\right)$ corresponding to zero eigenvalue is $v=\left(v_{1}, v_{2}, v_{3}, v_{4}\right)^{T}=$ $(0,0,1,0,0)$. Therefore,

$$
\begin{aligned}
a & =\sum_{k, i, j=1}^{n} v_{k} w_{i} w_{j} \frac{\partial^{2} f_{k}}{\partial x_{i} \partial x_{j}}\left(E_{0}\right)=v_{3} \sum_{i, j=1}^{5} w_{i} w_{j} \frac{\partial^{2} f_{3}}{\partial x_{i} \partial x_{j}}\left(E_{0}\right)= \\
& =2 w_{1} w_{3} \beta_{1}+2 w_{1} w_{4} \beta_{2}+2 w_{2} w_{3} \beta_{1}\left(1+\omega_{1} \beta_{1}^{\prime}\right)+2 w_{2} w_{4} \beta_{2}\left(1+\omega_{2} \beta_{2}^{\prime}\right)= \\
& =2\left[w_{1} \beta_{1}+w_{2} \beta_{1}\left(1+\omega_{1} \beta_{1}^{\prime}\right)\right] w_{3}+2\left[w_{1} \beta_{2}+w_{2} \beta_{2}\left(1+\omega_{2} \beta_{2}^{\prime}\right)\right] w_{4} .
\end{aligned}
$$




$$
\begin{aligned}
b & =\sum_{k, i=1}^{n} v_{k} w_{i} \frac{\partial^{2} f_{k}}{\partial x_{i} \partial \Phi}\left(E_{0}\right)=v_{3} \sum_{i=1}^{5} w_{i} \frac{\partial^{2} f_{3}}{\partial x_{i} \partial \beta_{1}}\left(E_{0}\right)= \\
& =w_{3}\left\{X_{10}^{*}+\left(1+\omega_{1} \beta_{1}^{\prime}\right) X_{20}^{*}\right\}= \\
& =d(d+\gamma)(d+\theta)(d+\delta+\alpha)\left\{X_{10}^{*}+\left(1+\omega_{1} \beta_{1}^{\prime}\right) X_{20}^{*}\right\}>0 .
\end{aligned}
$$

Now, applying the last condition of Theorem 8 , it is observed that the system possesses a forward bifurcation when $a<0$ and a backward bifurcation when $a>0$. So, we get the theorem as follows:

Theorem 9. If $a>0$, then system (1) undergoes a backward bifurcation at $R_{0}=1$, otherwise if $a<0$, then it undergoes a forward bifurcation and the endemic equilibrium is locally asymptotically stable for $R_{0}>1$ with the bifurcation parameter $\beta_{1}=\beta_{1}^{*}$.

\section{NUMERICAL SIMULATION WITHOUT ANY CONTROL POLICY}

This section contains the numerical simulation of model system (1) where no control interventions are applied. Let us fix most of the parameters in Table $\underline{1}$.

Table 1. Parameter values used for numerical simulation of system (1)

\begin{tabular}{|l|l|l|l|l|l|l|}
\hline \multicolumn{7}{|c|}{ Parametric Values } \\
\hline$\Lambda$ & $p$ & $d$ & $\theta$ & $q$ & $\beta_{2}$ & $\gamma$ \\
\hline 10 & 0.4 & 0.02 & 0.04 & 0.6 & 0.00005 & 0.04 \\
\hline \hline$\omega_{1}$ & $\omega_{2}$ & $\beta_{1}^{\prime}$ & $\beta_{2}^{\prime}$ & $\delta$ & $\eta$ & $\alpha$ \\
\hline 0.3 & 0.05 & 0.1 & 0.01 & 0.03 & 0.02 & 0.05 \\
\hline
\end{tabular}

Figure 2 shows that for $\beta_{1}=0.00002$, we have the basic reproduction number as $R_{0}=$ $0.22(<1)$ and the trajectory starting from $x_{0} \equiv(0.5,0.03,0.5,0.03,0.5)$ ultimately converges to $E_{0}(100,400,0,0,0)$ where only susceptible population and stressed population live as a steady state.

But for an increasing value of $\beta_{1}$ (i.e., $\left.\beta_{1}=0.0002\right), R_{0}=1.53(>1)$ and hence disease invade in the system resulting in occurrence of endemic equilibrium point. Figure $\underline{3}$ shows that the trajectory starting from the same initial point approaches to endemic equilibrium point $E^{*}(85.95,234.25,54.80,10.96,9.13)$ and $E_{0}$ becomes unstable in this situation.

So, it is noticed that from the steady state situation of $E_{0}$, if the disease transmission rate among asymptomatically infected individual starts to increase, then at a threshold value of $\beta_{1}, E_{0}$ loses its stability and becomes unstable. Figure $\underline{4}$ shows that the system without any control interventions undergoes a forward bifurcation specifically a transcritical bifurcation around $E_{0}$ at $\beta=\beta_{[T C]}=0.00017$.

Now $\theta$ denotes the rate at which susceptible population moves into stressed class due to environmental pollution and toxin. So, it is quite obvious that increasing level of pollution leads to move more people entering into stressed class. In Figure $\underline{5}$ we portray the same scenario. It is concluded from this figure that if $\theta$ starts to increase, then the number of people in stressed population class increases with time. 

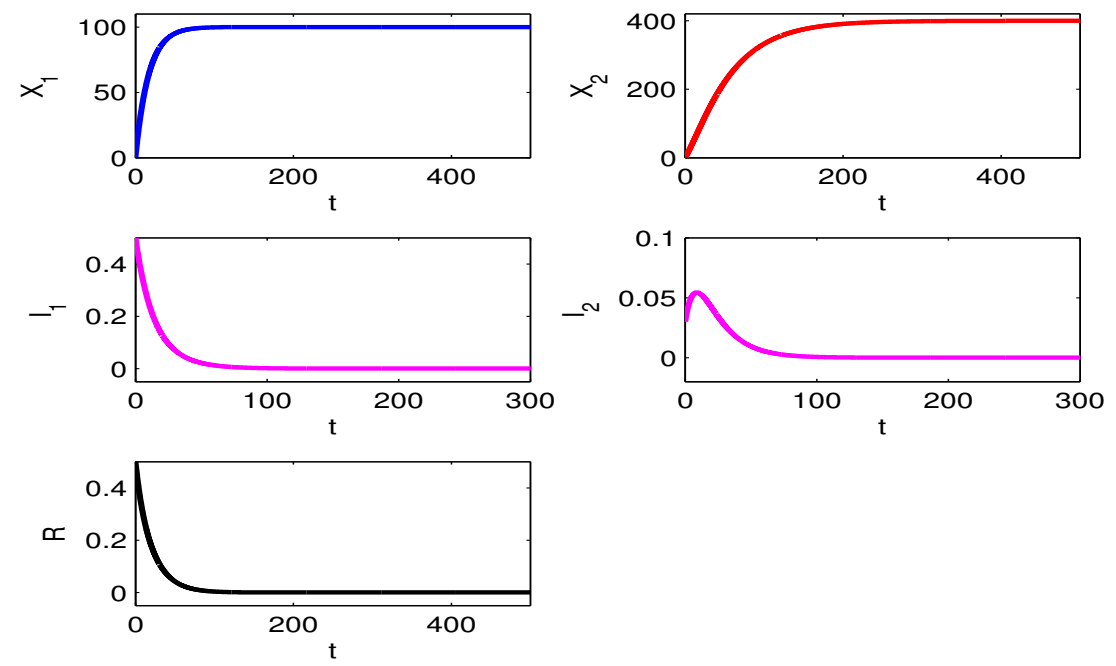

Fig. 2. Stability of the populations around disease-free equilibrium $E_{0}$.
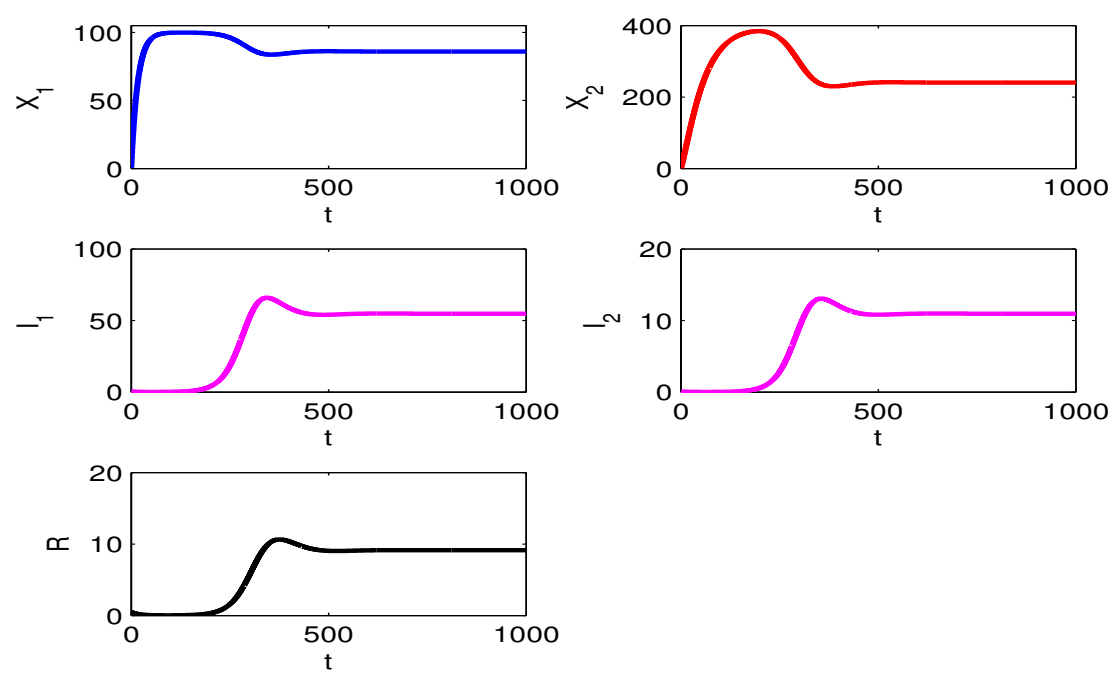

Fig. 3. Stability of the populations around endemic equilibrium $E^{*}$.

Figure 6 depicts the impact of $\theta$ on the stressed class more specifically. It is observed that the population in stressed class increases with a higher rate for smaller value of $\theta$. But for a comparatively higher value of $\theta$ (i.e., $\theta>0.3$ ), the curve loses its steepness and increases with a slower rate.

In Figure 7, the sensitivities of some of the parameters in disease transmission have been observed. The pictures depict that the transmission rate among asymptomatically infected individuals $\left(\beta_{1}\right)$ is most sensitive to control the reproduction number $\left(R_{0}\right)$. Besides of it, the transmission rate among symptomatically infected individual $\left(\beta_{2}\right)$, the disease transmission rate among asymptomatically infected individuals due to pollution $\left(\beta_{1}^{\prime}\right)$, one of the stress-related parameters $\left(\omega_{1}\right)$ and the rate at which susceptible moves to stressed class $(\theta)$ also increase with an increase of $R_{0}$ but the rate of increment is slower in these cases. Further $\beta_{2}^{\prime}$ and $\omega_{2}$ hardly participate to control $R_{0}$. On the other hand, the recovery rate $(\alpha)$ is inversely proportional to $R_{0}$ which is true, i.e., $R_{0}$ starts to increases as $\alpha$ decreases and the disease invade in the system.

Figure 8 shows how the rate at which susceptible moves to stressed class $(\theta)$ affects disease 


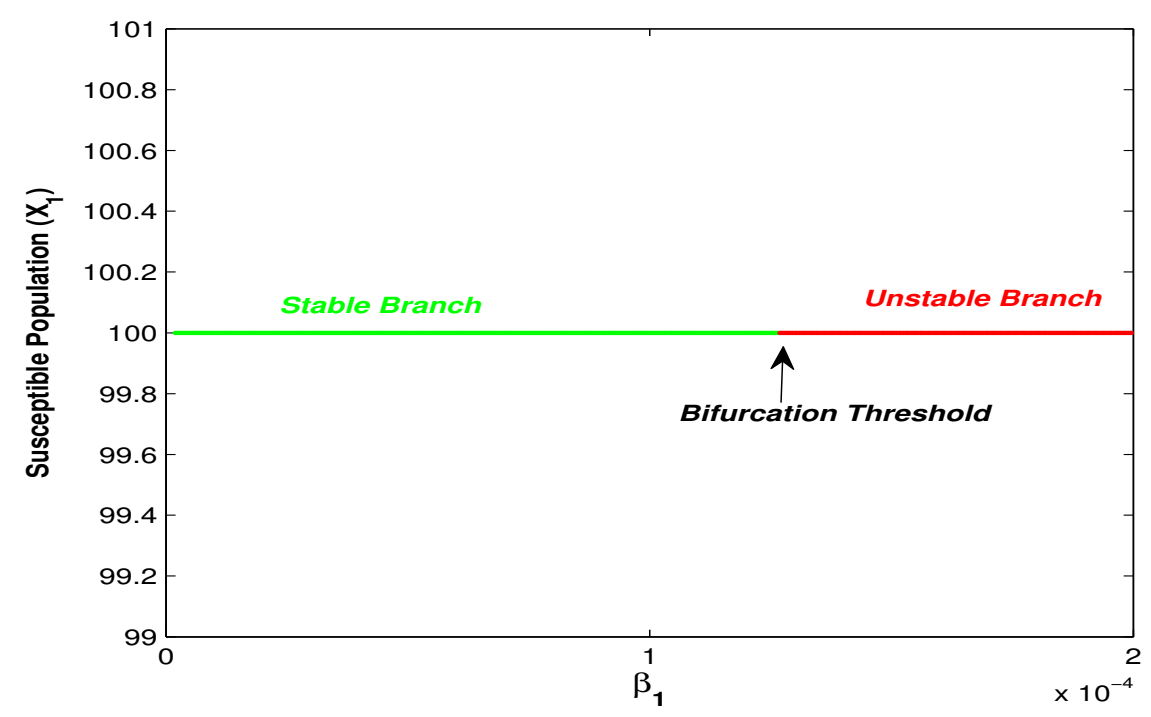

Fig. 4. Trancritical bifurcation around $E_{0}$ taking $\beta_{1}$ as bifurcation parameter.

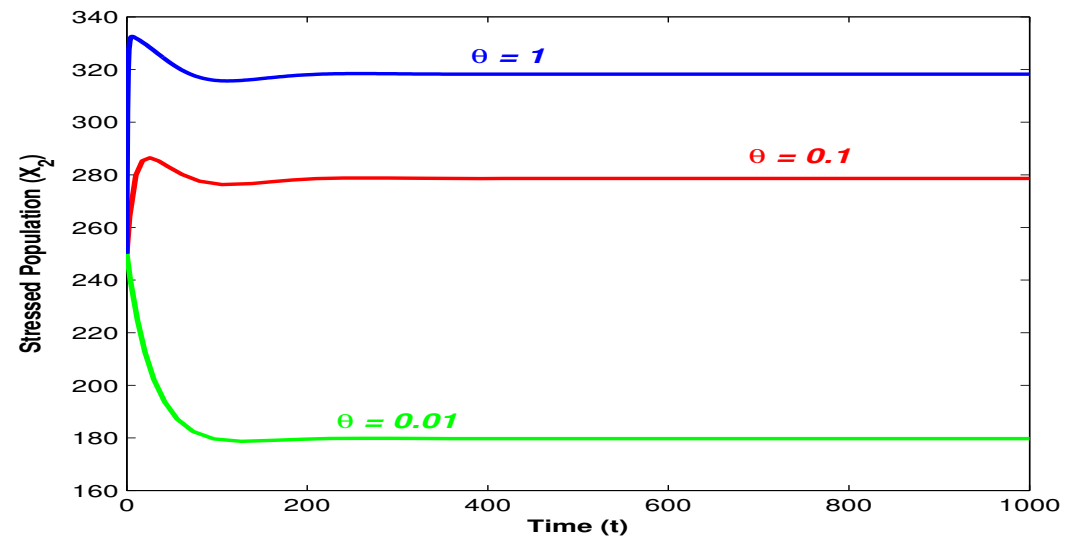

Fig. 5. Trajectory profiles of stressed population $\left(X_{2}\right)$ for different values of $\theta$.

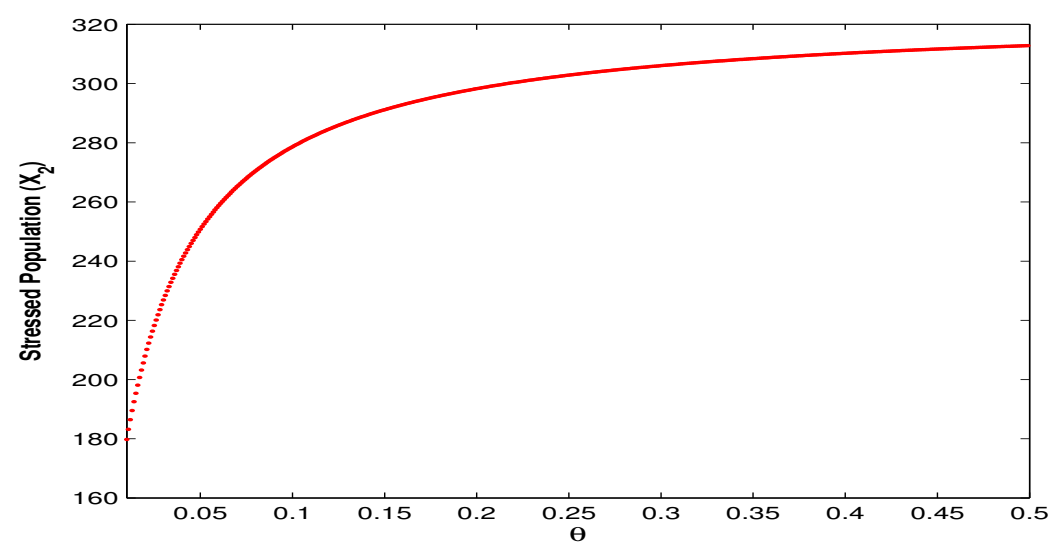

Fig. 6. Variation of steady state level of stressed population for different values of $\theta$.

transmission. From the figure, it is concluded that increasing value of $\theta$ leads to the increase of asymptomatically infected individuals $\left(I_{1}\right)$. If more population enters into stressed class, then the disease can be transmitted easily and we get more of asymptomatically infected individuals. 


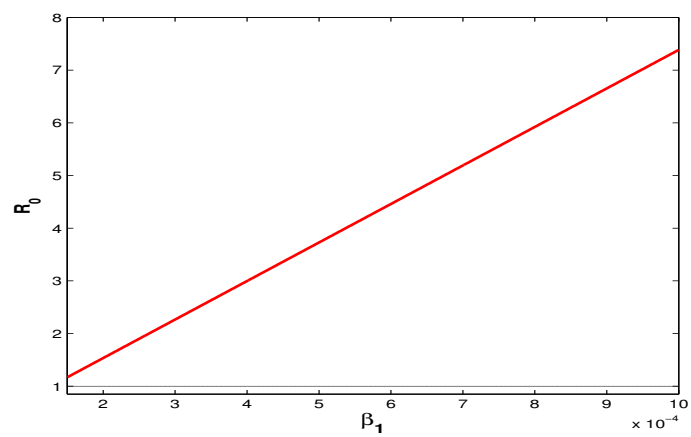

(a)

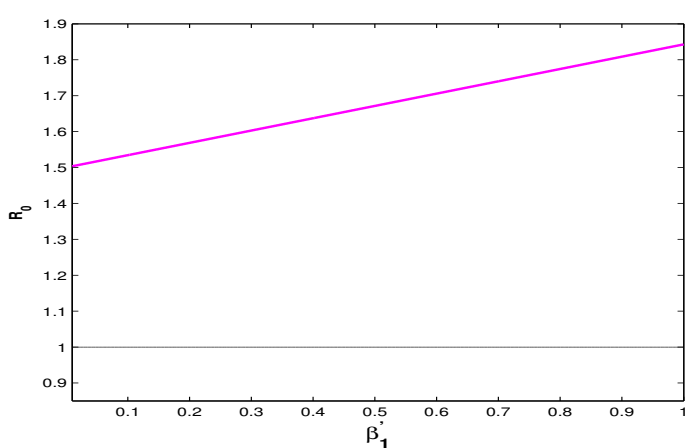

(c)

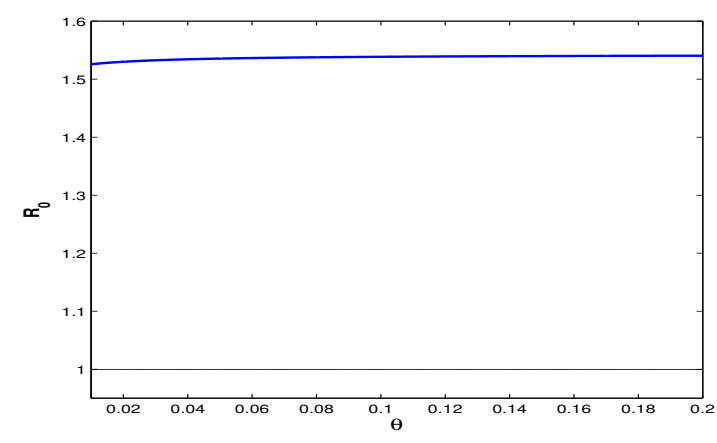

(e)

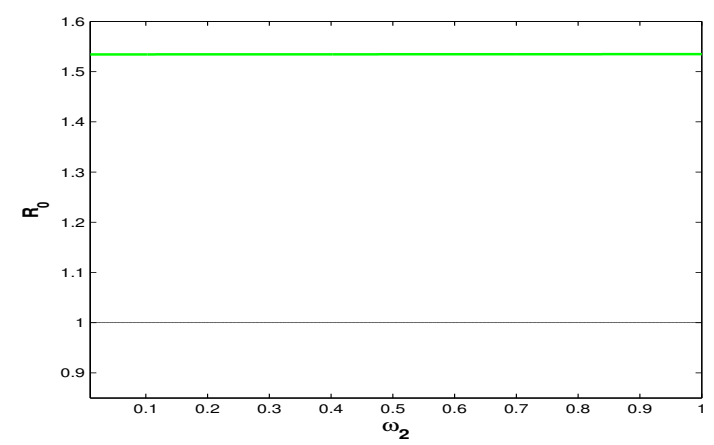

(g)

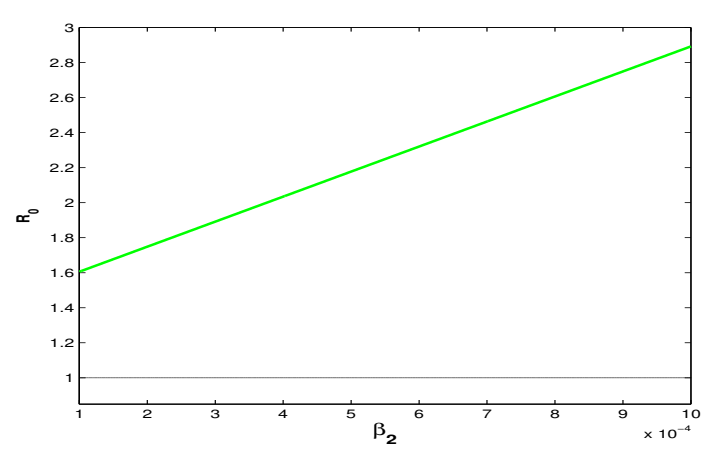

(b)

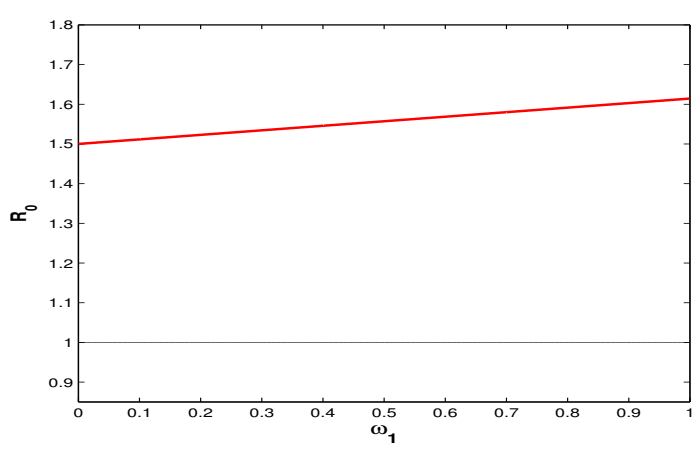

(d)

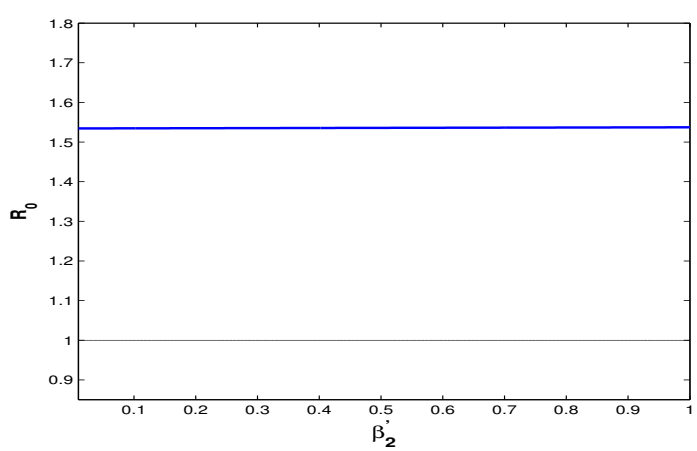

(f)

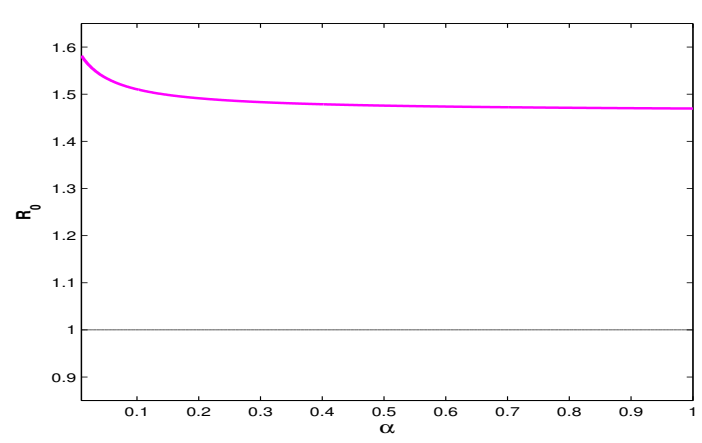

(h)

Fig. 7. Relationship between basic reproduction number $R_{0}$ with $\beta_{1}, \beta_{2}, \beta_{1}^{\prime}, \omega_{1}, \theta, \beta_{2}^{\prime}, \omega_{2}$ and $\alpha$.

In Figure 9, we have plotted the asymptomatically infected individuals against of stress-related parameters $\left(\omega_{1}, \omega_{2}\right)$ to observe the variation of $I_{1}$ over time for different values of $p$. In this figure, it is shown that the infected population increases when $p$ starts to increase. In 


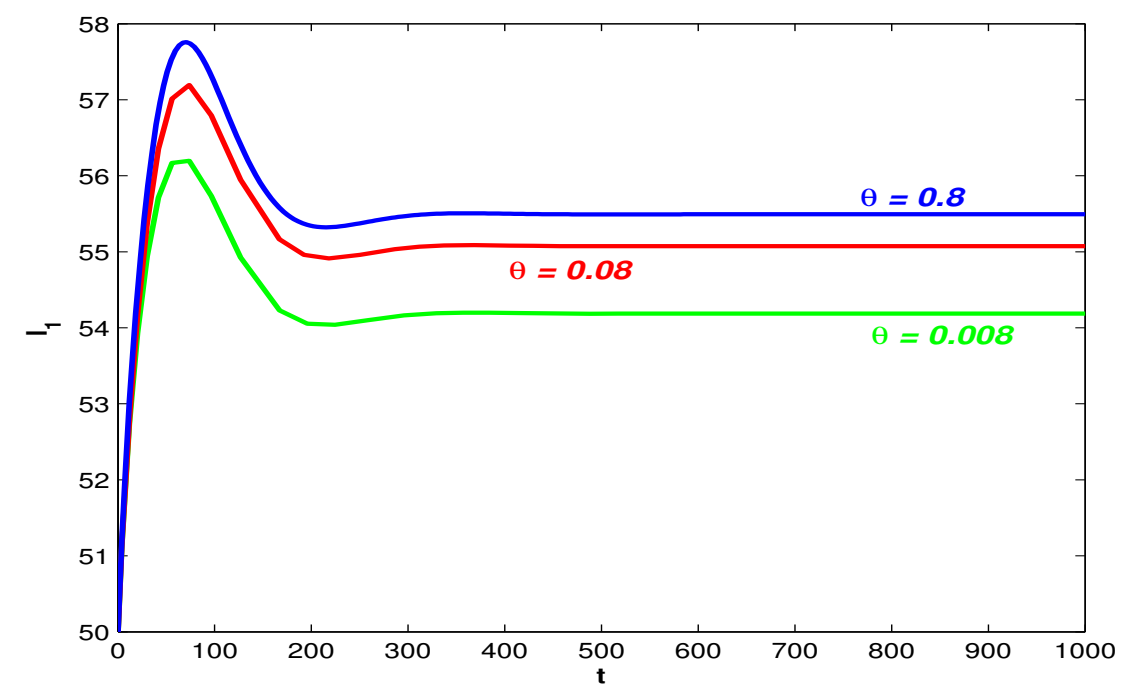

Fig. 8. Trajectory profiles of asymptomatically infected population $\left(I_{1}\right)$ for different values of $\theta$.

Figure 9,a the rate of increment is higher than of Figure 9,b, i.e., $\omega_{1}$ has more impact on disease transmission than $\omega_{2}$. It is also concluded that the higher level of environmental stress can cause an increase in disease transmission.

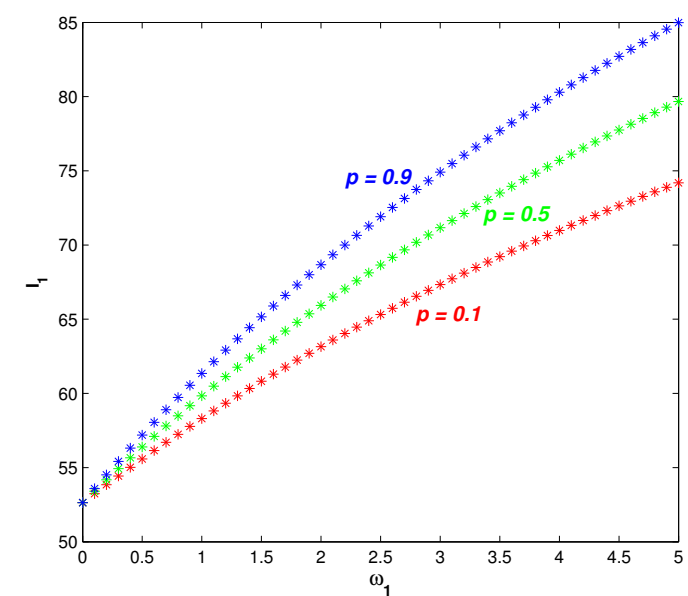

(a)

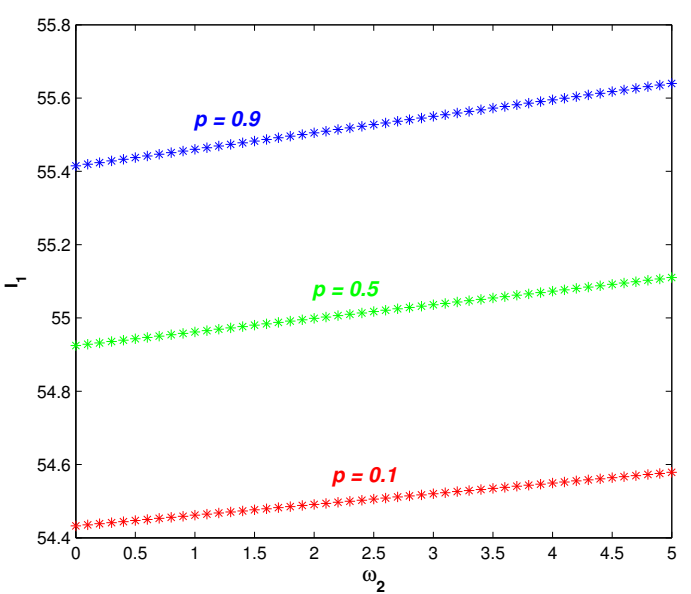

(b)

Fig. 9. (a) Variation of asymptomatically infected population $\left(I_{1}\right)$ due to change in pollution level, $\omega_{1}$ for different values of $p$. (b) Variation of asymptomatically infected population $\left(I_{1}\right)$ due to change in pollution level, $\omega_{2}$ for different values of $p$.

Also, infected population changes for increasing stress levels $\left(\beta_{1}^{\prime}, \beta_{2}^{\prime}\right)$ with increasing value of $\theta$ (see Figure 10). In figures 11 and 12, the impacts of stress related parameters $\omega_{1}, \omega_{2}, \beta_{1}^{\prime}, \beta_{2}^{\prime}$ on asymptomatically infected individual have been studied for different values of $\theta$ and $p$ respectively. From Figure 9 - Figure 12, it is concluded that the asymptomatically infected individuals increase with $\omega_{i}, \boldsymbol{\beta}_{i}^{\prime}$ for $i=\overline{1}, 2$ when $\theta$ and $p$ are increased. As a result, the environmental pollution has an important role in disease transmission.

In Figure 13, the growth of asymptomatically infected individuals has been observed in presence as well as in absence of stressed population. In the case of absence of stressed population, we have taken $p=0, \theta=0$ and $q=0$ to stop the influx of people in $X_{2}$ class. It is observed that the growth of asymptomatically infected population is lower when there is no 


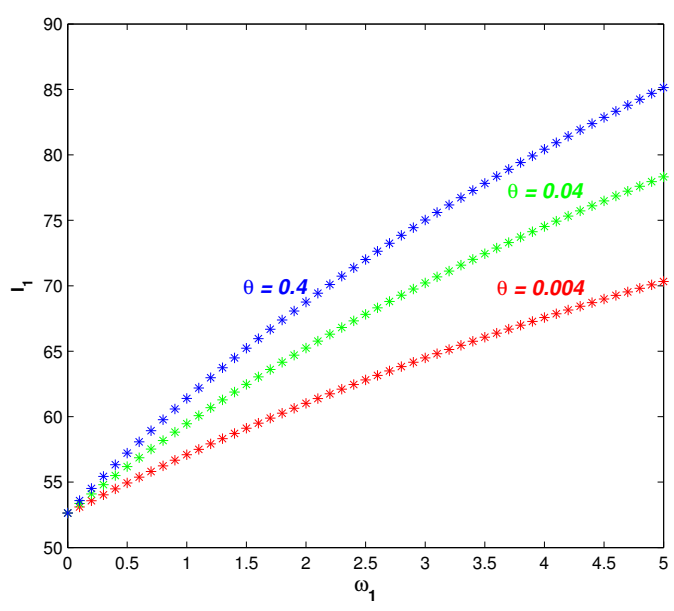

(a)

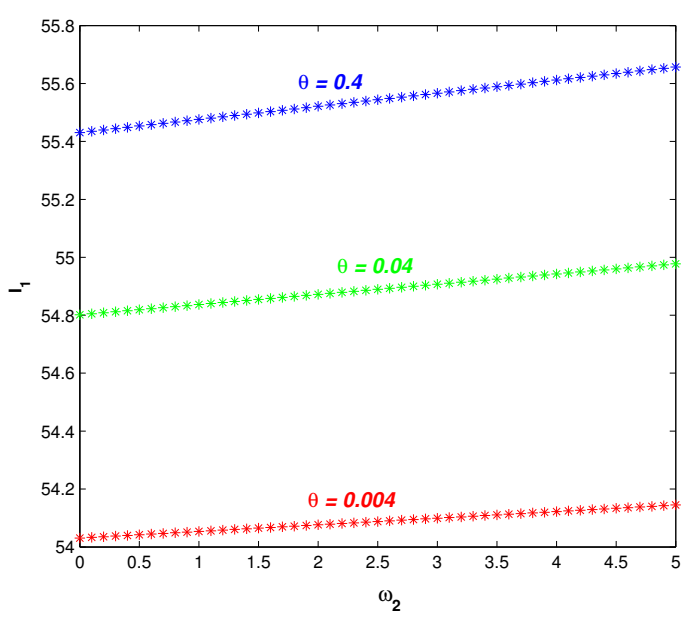

(b)

Fig. 10. (a) Variation of asymptomatically infected population $\left(I_{1}\right)$ due to change in pollution level, $\omega_{1}$ for different values of $\theta$. (b) Variation of asymptomatically infected population $\left(I_{1}\right)$ due to change in pollution level, $\omega_{2}$ for different values of $\theta$.

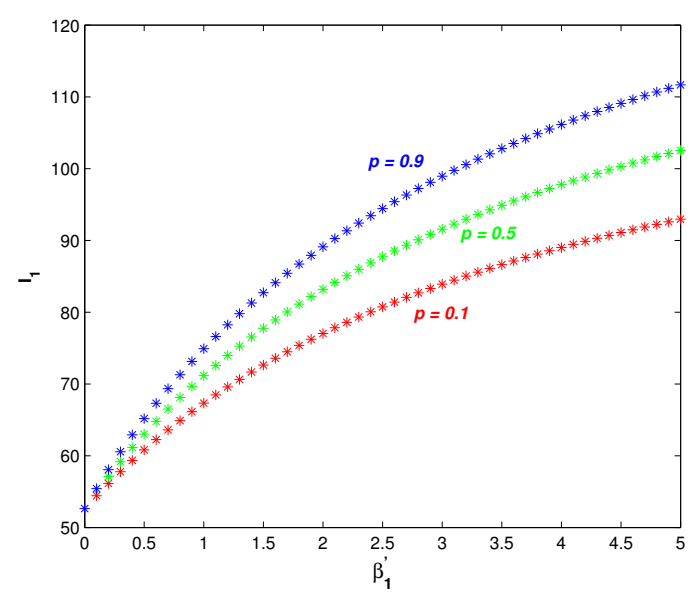

(a)

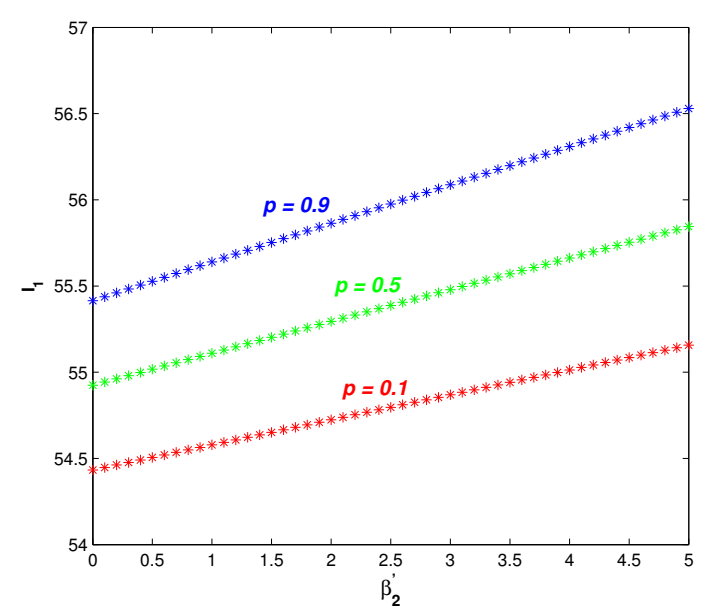

(b)

Fig. 11. (a) Variation of asymptomatically infected population $\left(I_{1}\right)$ due to $\beta_{1}^{\prime}$, which is effect of pollution on $\beta_{1}$ for different values of $p$. (b) Variation of asymptomatically infected population ( $\left.I_{1}\right)$ due to $\beta_{2}^{\prime}$, which is effect of pollution on $\beta_{2}$ for different values of $p$. 


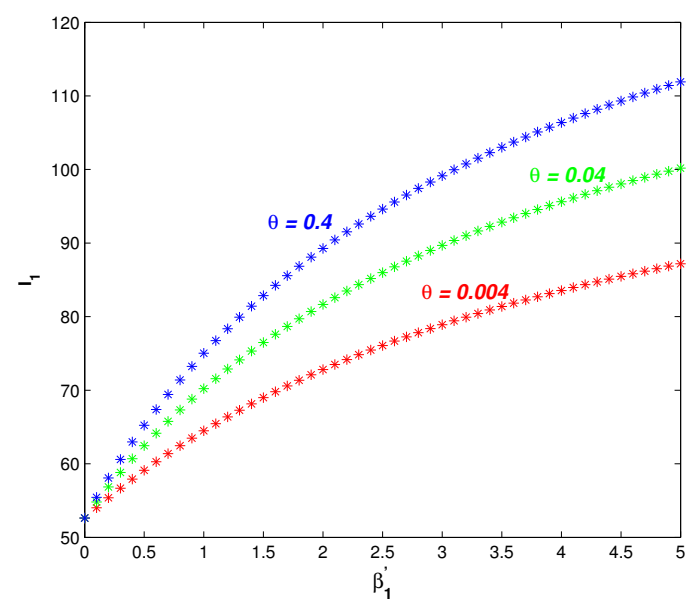

(a)

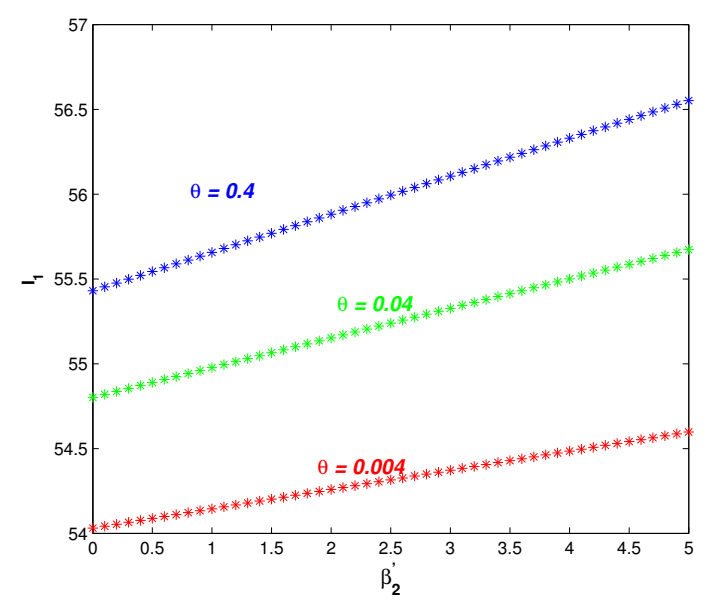

(b)

Fig. 12. (a) Variation of asymptomatically infected population $\left(I_{1}\right)$ due to $\beta_{1}^{\prime}$, which is effect of pollution on $\beta_{1}$ for different values of $\theta$. (b) Variation of asymptomatically infected population $\left(I_{1}\right)$ due to $\beta_{2}^{\prime}$, which is effect of pollution on $\beta_{2}$ for different values of $\theta$.

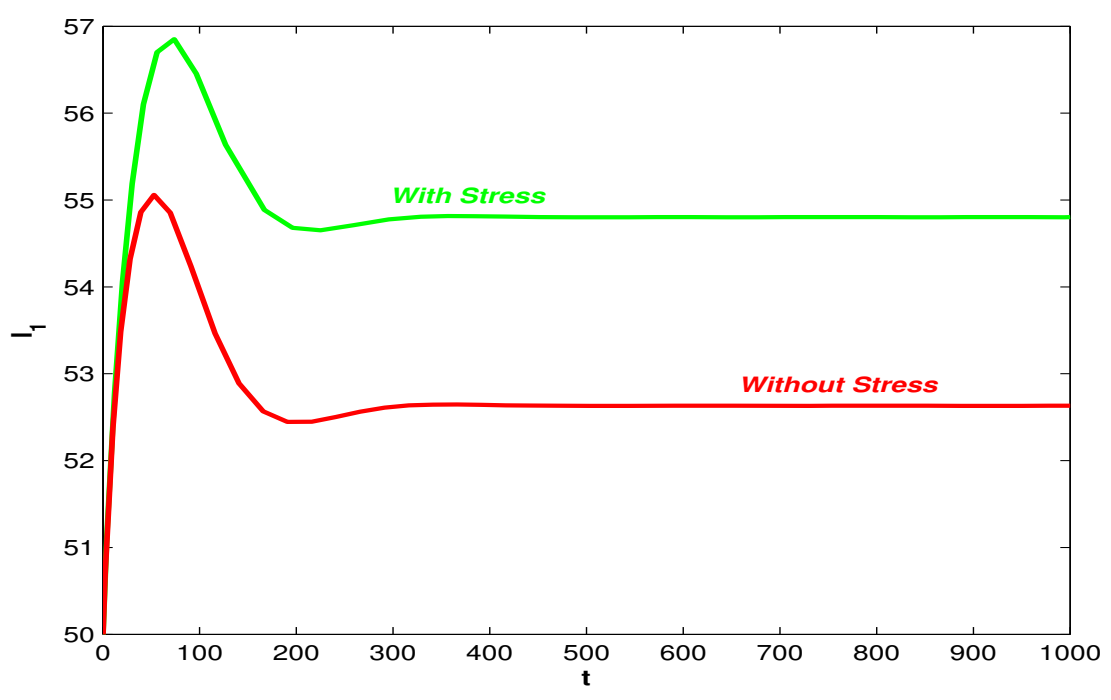

Fig. 13. Trajectories of asymptomatically infected population in the presence and absence of stress.

environmental stress.

\section{OPTIMAL CONTROL PROBLEM}

Here we have formulated an optimal control problem corresponding to system (1) taking (i) treatment policy to symptomatically infected individuals and (ii) vaccination policies for susceptible and stressed people as control strategies. We have analysed how these control parameters make an impact on disease transmission and also try to optimize the cost incurred in their implementations [47, 48, 49]. A brief description about these control policies will help to understand their influence for controlling the disease transmission.

(i) Providing treatment to symptomatically infected individuals: More of infectious people enter into recovered class if proper and better treatment can be given to them at symptomatically infected stage. So, a better treatment can low the disease prevalence resulting in a lesser infected 
present in the system and hence the transmission from susceptible and stressed classes to infected class also reduce. Now, medical treatments or diagnosis provided to patients are limited and this is why we have incorporated a saturated treatment rate function $\frac{\epsilon_{3} u_{3} I_{2}}{1+\zeta_{3} I_{2}}$ to obtain (7). Here $\epsilon_{3}$ denotes the treatment rate with intensity $u_{3}$ and saturation constant $\zeta_{3}^{-1}$. All the expenses regarding diagnosis procedure have been considered at the time of treatment. Also, the treatment intensity $u_{3}$ is the control variable with the restriction $0 \leq u_{3} \leq 1$.

(ii) Providing vaccines to susceptible and stressed individuals: Proper vaccination given to susceptible and stressed population acts as a precaution from getting infected. If we can introduce some vaccination processes for both the susceptible and stressed individuals so that they can directly move into recovered class, then that can be considered as a possible tool to decrease the disease transmission in the population. In our model system (7), $u_{1}, u_{2}$ represent the intensities of vaccination processes satisfying $0 \leq u_{1} \leq 1,0 \leq u_{2} \leq 1$ where 0 implies no improvement by vaccine and 1 implies full improvement by proper vaccine. Also, $\epsilon_{1}, \epsilon_{2}$ denote the rates at which a vaccine is provided to the individuals. So, both $u_{1}$ and $u_{2}$ change with vaccination procedure which implies that $u_{1}, u_{2}$ can be taken as control variables. As this process requires some external bits of help and is not natural, so, vaccine cost etc. are involved in terms of non-linear functions of $u_{1}(t), u_{2}(t)$ to enhance the procedure.

The main aim of formulating a system with control interventions is to obtain optimal treatment as well as optimal vaccination intensity by dint of information with minimum cost. The mentioned analysis gives that the control variables $u_{1}(t), u_{2}(t)$ and $u_{3}(t)$ belong to the following set:

$$
\Pi=\left\{\left(u_{1}(t), u_{2}(t), u_{3}(t)\right) \mid\left(u_{1}(t), u_{2}(t), u_{3}(t)\right) \in[0,1] \times[0,1] \times[0,1], t \in\left[0, T_{f}\right]\right\}
$$

where $T_{f}$ is the final time up to which control policies can be executed, and $u_{1}(t), u_{2}(t)$ and $u_{3}(t)$ all are bounded and measurable functions.

\subsection{Determination of Total Cost}

Let us first determine the total cost that has to be minimized for control interventions in the system.

(i) Cost involved in treatment to symptomatically infected individuals: The total cost associated with disease burden and better treatment for symptomatic infected people is

$$
\int_{0}^{T_{f}}\left[w_{1} I_{2}(t)+w_{4} u_{3}^{2}(t)\right] d t
$$

Cost associated with symptomatically infected people due to loss in manpower and the wealth is represented by $w_{1} I_{2}(t)[\underline{50}, \underline{51}, \underline{52}]$ which expresses the opportunity loss or productivity lose due to sickness. The term $w_{4} u_{3}^{2}$ denotes the cost during treatment including medication charges, the better expenditure of hospitalization etc. For treatment policy, we have considered a quadratic nonlinearity $u_{3}^{2}[\underline{50}, \underline{51}, \underline{52}]$. Non-linearity up to $2^{\text {nd }}$ order represents the seriousness of the drugs which are used during the treatment period. Here $w_{1}, w_{4}$ are the positive weights associated with symptomatically infected people and better treatment policy respectively.

(ii) Cost involved in vaccination procedure: The total cost incurred for the vaccination process given to susceptible and stressed population is

$$
\int_{0}^{T_{f}}\left[w_{2} u_{1}^{2}(t)+w_{3} u_{2}^{2}(t)\right] d t
$$


Here $w_{2} u_{1}^{2}(t)$ and $w_{3} u_{3}^{2}(t)$ denote the costs for vaccines applied to susceptible and stressed population respectively. By convention, $w_{2}, w_{3}$ are the positive weights associated with vaccination. These terms include the costs of the whole procedure of vaccination. Now, all the vaccines are not easily available and also even affordable. So, applying such vaccines are costly enough. Some researchers, in their work, have analysed the impact of the cost associated considering the non-linearity up to order two [포 $\underline{51}, \underline{54}]$ and we have taken the same assumption here. The non-linearity up to order two has been taken here as $u_{1}^{2}(t)$ and $u_{2}^{2}(t)$ which describe the impact of the cost associated with vaccination. These terms reflect the importance of vaccination to reduce disease transmission also.

The following control problem is constructed taking into consideration the previous discussions along with the cost functional $J$ which has to be minimized:

$$
J\left[u_{1}(t), u_{2}(t), u_{3}(t)\right]=\int_{0}^{T_{f}}\left[w_{1} I_{2}(t)+w_{2} u_{1}^{2}(t)+w_{3} u_{2}^{2}(t)+w_{4} u_{3}^{2}(t)\right] d t
$$

subject to the model system:

$$
\begin{aligned}
\frac{d X_{1}}{d t} & =(1-p) \Lambda-d X_{1}-\theta X_{1}+(1-q) \gamma R-\left(\beta_{1} I_{1}+\beta_{2} I_{2}\right) X_{1}-\frac{\epsilon_{1} u_{1} X_{1}}{1+\zeta_{1} X_{1}}, \\
\frac{d X_{2}}{d t} & =p \Lambda+\theta X_{1}-d X_{2}+q \gamma R-\left\{\beta_{1}\left(1+\omega_{1} \beta_{1}^{\prime}\right) I_{1}+\beta_{2}\left(1+\omega_{2} \beta_{2}^{\prime}\right) I_{2}\right\} X_{2}-\frac{\epsilon_{2} u_{2} X_{2}}{1+\zeta_{2} X_{2}}, \\
\frac{d I_{1}}{d t} & =\left(\beta_{1} I_{1}+\beta_{2} I_{2}\right) X_{1}+\left\{\beta_{1}\left(1+\omega_{1} \beta_{1}^{\prime}\right) I_{1}+\beta_{2}\left(1+\omega_{2} \beta_{2}^{\prime}\right) I_{2}\right\} X_{2}-(d+\delta+\eta) I_{1}, \\
\frac{d I_{2}}{d t} & =\eta I_{1}-(d+\delta) I_{2}-\alpha I_{2}-\frac{\epsilon_{3} u_{3} I_{2}}{1+\zeta_{3} I_{2}}, \\
\frac{d R}{d t} & =\alpha I_{2}-(\gamma+d) R+\frac{\epsilon_{1} u_{1} X_{1}}{1+\zeta_{1} X_{1}}+\frac{\epsilon_{2} u_{2} X_{2}}{1+\zeta_{2} X_{2}}+\frac{\epsilon_{3} u_{3} I_{2}}{1+\zeta_{3} I_{2}},
\end{aligned}
$$

with the initial conditions $X_{1}(0)>0, X_{2}(0)>0, I_{1}(0) \geq 0, I_{2}(0) \geq 0$ and $R(0) \geq 0$. Here the functional $J$ is the total cost representing sum of the costs as stated. The integrand:

$$
L\left(X_{1}, X_{2}, I_{1}, I_{2}, R, u_{1}(t), u_{2}(t), u_{3}(t)\right)=w_{1} I_{2}(t)+w_{2} u_{1}^{2}(t)+w_{3} u_{2}^{2}(t)+w_{4} u_{3}^{2}(t)
$$

denotes the cost at time $t$. Parameters $w_{1}, w_{2}, w_{3}$ and $w_{4}$ are positive weight constants which balance the units of integrand $[\underline{50}, \underline{51}]$. Let us denote $u_{1}(t)=u_{1}, u_{2}(t)=u_{2}$ and $u_{3}(t)=u_{3}$ at the time of calculation. The optimal control interventions $u_{1}^{*}, u_{2}^{*}$ and $u_{3}^{*}$ exists in $\Pi$ and it mainly minimizes the cost functional $J$.

Theorem 10. There exists optimal controls $u_{1}^{*}, u_{2}^{*}$ and $u_{3}^{*}$ in $\Pi$ corresponding to the control system (6)-(7) such that $J\left(u_{1}^{*}, u_{2}^{*}, u_{3}^{*}\right)=\min \left[J\left(u_{1}, u_{2}, u_{3}\right)\right]$.

Pr o of. Proof has been given in Appendix.

Now, by Pontryagin's Maximum Principle optimal controls $u_{1}^{*}, u_{2}^{*}$ and $u_{3}^{*}$ of the system can be found as follows.

Theorem 11. If $u_{1}^{*}, u_{2}^{*}$ and $u_{3}^{*}$ be optimal control variables and $X_{1}^{*}, X_{2}^{*}, I_{1}^{*}, I_{2}^{*}, R^{*}$ be the corresponding optimal state variables of the control system (6)-(7), then there exists adjoint 
variable $\lambda=\left(\lambda_{1}, \lambda_{2}, \lambda_{3}, \lambda_{4}, \lambda_{5}\right) \in \mathbb{R}^{5}$ which satisfies the following canonical equations:

$$
\begin{aligned}
\frac{d \lambda_{1}}{d t} & =\lambda_{1}\left[d+\theta+\beta_{1} I_{1}+\beta_{2} I_{2}+\frac{\epsilon_{1} u_{1}}{\left(1+\zeta_{1} X_{1}\right)^{2}}\right]-\lambda_{2} \theta-\lambda_{3}\left(\beta_{1} I_{1}+\beta_{2} I_{2}\right)-\lambda_{5} \frac{\epsilon_{1} u_{1}}{\left(1+\zeta_{1} X_{1}\right)^{2}} \\
\frac{d \lambda_{2}}{d t} & =\lambda_{2}\left[d+\beta_{1}\left(1+\omega_{1} \beta_{1}^{\prime}\right) I_{1}+\beta_{2}\left(1+\omega_{2} \beta_{2}^{\prime}\right) I_{2}+\frac{\epsilon_{2} u_{2}}{\left(1+\zeta_{2} X_{2}\right)^{2}}\right] \\
& -\lambda_{3}\left[\beta_{1}\left(1+\omega_{1} \beta_{1}^{\prime}\right) I_{1}+\beta_{2}\left(1+\omega_{2} \beta_{2}^{\prime}\right) I_{2}\right]-\lambda_{5} \frac{\epsilon_{2} u_{2}}{\left(1+\zeta_{2} X_{2}\right)^{2}}, \\
\frac{d \lambda_{3}}{d t} & =\lambda_{1} \beta_{1} X_{1}+\lambda_{2} \beta_{1}\left(1+\omega_{1} \beta_{1}^{\prime}\right) X_{2}-\lambda_{3}\left\{\beta_{1} X_{1}+\beta_{1}\left(1+\omega_{1} \beta_{1}^{\prime}\right) X_{2}-(d+\delta+\eta)\right\}-\lambda_{4} \eta \\
\frac{d \lambda_{4}}{d t} & =-w_{1}+\lambda_{1} \beta_{2} X_{1}+\lambda_{2} \beta_{2}\left(1+\omega_{2} \beta_{2}^{\prime}\right) X_{2}-\lambda_{3}\left\{\beta_{2} X_{1}+\beta_{2}\left(1+\omega_{2} \beta_{2}^{\prime}\right) X_{2}\right\} \\
& +\lambda_{4}\left(d+\delta+\alpha+\frac{\epsilon_{3} u_{3}}{\left(1+\zeta_{3} I_{2}\right)^{2}}\right)-\lambda_{5}\left(\alpha+\frac{\epsilon_{3} u_{3}}{\left(1+\zeta_{3} I_{2}\right)^{2}}\right) \\
\frac{d \lambda_{5}}{d t} & =-\lambda_{1}(1-q) \gamma-\lambda_{2} q \gamma+\lambda_{5}(d+\gamma)
\end{aligned}
$$

with the transversality conditions $\lambda_{i}\left(T_{f}\right)=0$ for $i=1,2,3,4,5$. The corresponding optimal controls $u_{1}^{*}, u_{2}^{*}$ and $u_{3}^{*}$ are given by

$$
\begin{aligned}
u_{1}^{*} & =\min \left\{\max \left\{0,\left(\frac{\epsilon_{1} X_{1}^{*}}{2 w_{2}\left(1+\zeta_{1} X_{1}^{*}\right)}\left(\lambda_{1}-\lambda_{5}\right)\right)\right\}, 1\right\}, \\
u_{2}^{*} & =\min \left\{\max \left\{0,\left(\frac{\epsilon_{2} X_{2}^{*}}{2 w_{3}\left(1+\zeta_{2} X_{2}^{*}\right)}\left(\lambda_{2}-\lambda_{5}\right)\right)\right\}, 1\right\}, \\
\text { and } \quad u_{3}^{*} & =\min \left\{\max \left\{0,\left(\frac{\epsilon_{3} I_{2}^{*}}{2 w_{4}\left(1+\zeta_{3} I_{2}^{*}\right)}\left(\lambda_{4}-\lambda_{5}\right)\right)\right\}, 1\right\} .
\end{aligned}
$$

Pro of. Proof is given in Appendix.

\section{NUMERICAL SIMULATION IMPLEMENTING THE CONTROL POLICIES}

In previous sections, we have analyzed the stability criterion of the equilibrium points and found the optimal control paths to minimize the total cost considered for corresponding optimal control problem. Here we have performed the numerical simulations for system (7) to support the analytical findings and to observe the effect of control variables in the system dynamics. We have considered the cases of implementation of one or two or all control strategies to find the minimal cost one by one.

The corresponding control system stated in equations (6)-(7) have been solved here numerically for parametric values in Table 2 with the initial population size: $X_{1}(0)=$ $90, X_{2}(0)=250, I_{1}(0)=50, I_{2}(0)=20$ and $R(0)=10$. The pictorial scenarios for different cases can be obtained by MATLAB. The optimal control interventions are found using Forward-backward sweep method. Here the optimal state system and the adjoint state system are respectively solved forward and backward in time. In the next step, the steepest descent method is used to update the optimal controls using Hamiltonian for the optimality of the system [60] and it continues till the convergence. In this work, the control policies are applied and observed for approximately 90 days.

Figure 14 shows the population trajectories with time when there are no control policies, i.e., $u_{i}=\overline{0}$ for $i=1,2,3$. In this situation, at $T_{f}=90$, the population is (85.16441616, 230.30053701, 57.07727059, 11.73755345, 10.32798982). It is observed that the growth of asymptomatically infective population first increases and reaches its maximum values within 
Table 2. Parametric values used in model system (7)

\begin{tabular}{|c|c|c|}
\hline Parameter & Value & Source \\
\hline$\Lambda$ & 10 person/day & {$[\underline{55]}$} \\
$\delta$ & $0.03 /$ day & {$[\underline{50]}$} \\
$d$ & 0.02 & {$[\underline{56}]$} \\
$\alpha$ & $0.05 /$ day & $\underline{[57]}$ \\
$\beta_{1}$ & $0.0002 /$ day & $\underline{[58]}$ \\
$\gamma$ & $0.04 /$ day & $\underline{[59]}$ \\
$\beta_{2}$ & $0.00005 /$ day & Assumed \\
$p$ & 0.4 & Assumed \\
$\theta$ & 0.04 & Assumed \\
$q$ & 0.6 & Assumed \\
$\omega_{1}$ & 0.3 & Assumed \\
$\omega_{2}$ & 0.05 & Assumed \\
$\beta_{1}^{\prime}$ & 0.1 & Assumed \\
$\beta_{2}^{\prime}$ & 0.01 & Assumed \\
$\eta$ & $0.02 /$ day & Assumed \\
$\epsilon_{1}$ & $0.001 /$ day & Assumed \\
$\epsilon_{2}$ & $0.8 /$ day & {$[\underline{50]}$} \\
$\epsilon_{3}$ & $0.8 /$ day & {$[\underline{50]}$} \\
$\zeta_{1}$ & $0.75 /$ day & Assumed \\
$\zeta_{2}$ & $0.045 /$ day & Assumed \\
$\zeta_{3}$ & $0.001 /$ day & Assumed \\
\hline
\end{tabular}

two weeks but then slowly decreases after around one month. Now, there is a significant number of infective present for more than 30 days which causes the economic burden in terms of productivity loss, mortality, morbidity and in procuring protective measures during this period.

In the following three figures the implementation effect of single control policy has been discussed where the positive weight constants have been taken as $w_{1}=1, w_{2}=500, w_{3}=$ 500 and $w_{4}=1800$ [50]. In Figure 15, the population trajectories have been drawn when the vaccination policy is applied only on susceptible individuals as a precaution, i.e., $u_{1}$ is taken to be a control parameter and the optimality of the system has been determined (taking $u_{2}=u_{3}=$ $0)$. At $T_{f}=90$, the population is $(85.16441508,230.30053695,57.07727047,11.73755344$, 10.32799112). It is observed that the recovered population becomes higher than the case when $u_{1}$ is not implemented. If the people get vaccinated at the susceptible stage, then the chances of being infected are lower and in fact, the count of people in both infectious stages has reduced in this case. The top figure of left column in Figure 18 depicts the path of optimal intensity which 

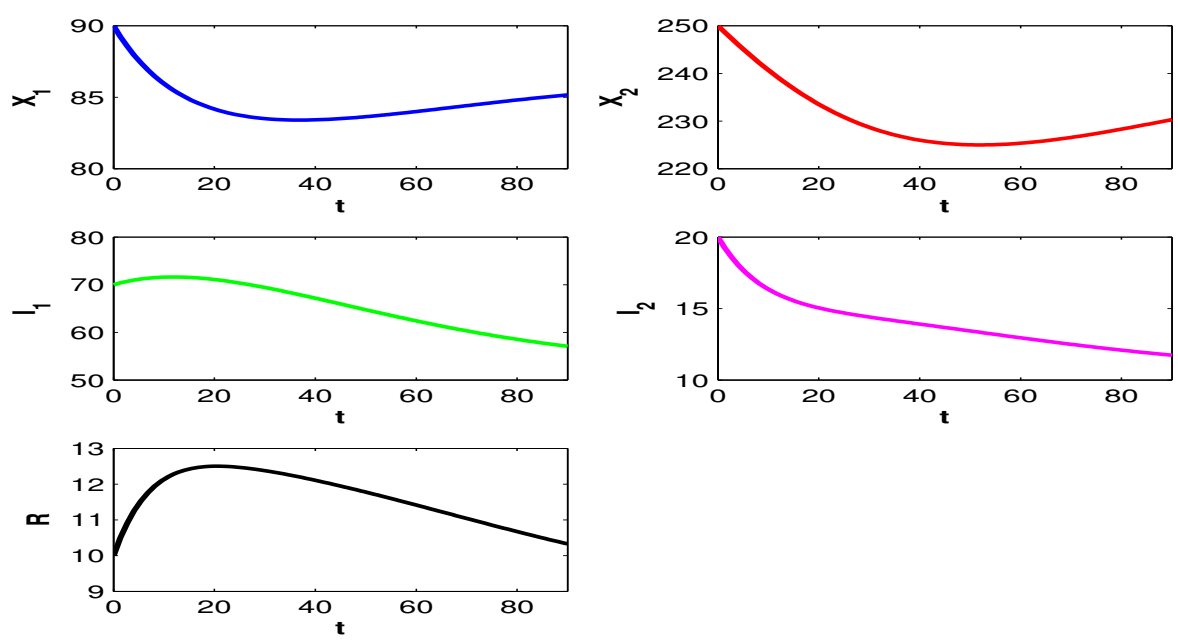

Fig. 14. Profiles of populations in absence of controls. Parameters are as in Table 2.
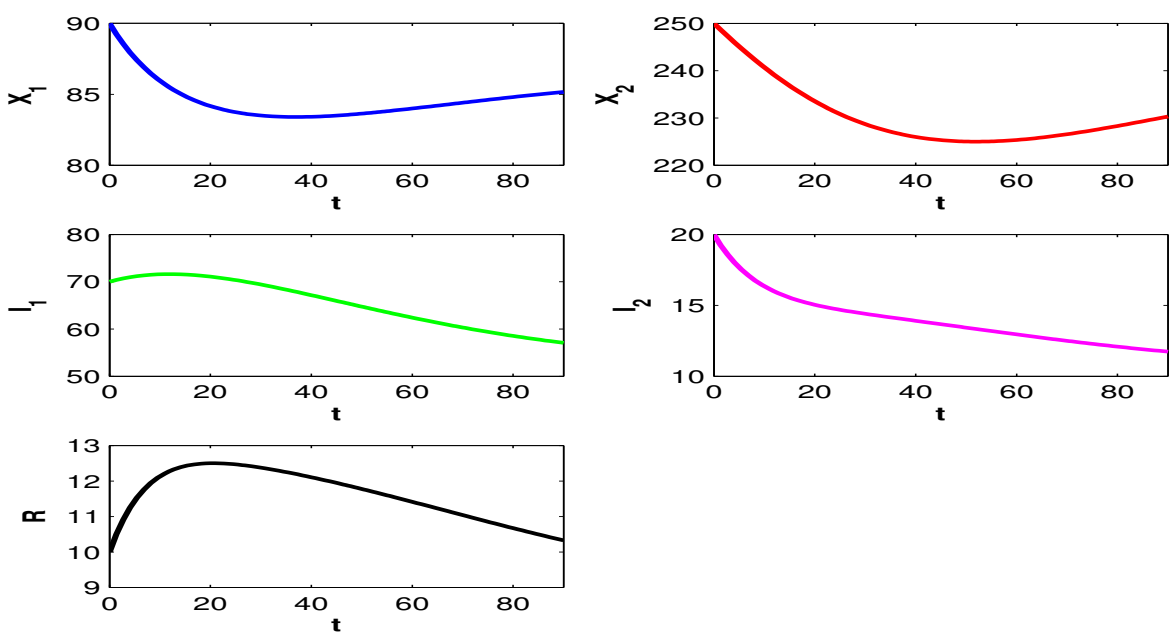

Fig. 15. Profiles of populations with applied optimal treatment $u_{1}^{*}$ only and $u_{2}=u_{3}=0$.

represents the pharmaceutical vaccination provided to the susceptible population. It shows that this particular vaccination strategy hardly makes any impact in earlier days but after almost two and a half months the intensity becomes higher. It leads to the conclusion that the applied vaccination needs some time to show its effect to control the disease burden.

The population trajectories in Figure 16 can be obtained by solving system (7) only when the vaccination applied to stressed population $\left(u_{2}\right)$ is considered. Here at $T_{f}=90$, the population is (85.1681878, 230.34242211, 57.07635690, 11.73587789, 10.32661119). Parameters have been taken from Table 2 with mentioned weights. Vaccination provided to stressed population works comparatively better to control the disease transmission as it increases both susceptible and stressed population but decreases both infected populations. The right side figure in Figure 18 depicts the path of optimal intensity which represents the pharmaceutical vaccination provided to the stressed population. In this case, it is observed that this particular vaccination strategy works with a higher rate at an earlier stage of infection but after almost one and a half month, the intensity becomes almost zero. It leads to the conclusion that the applied vaccination has a transient effect to control the disease burden. 

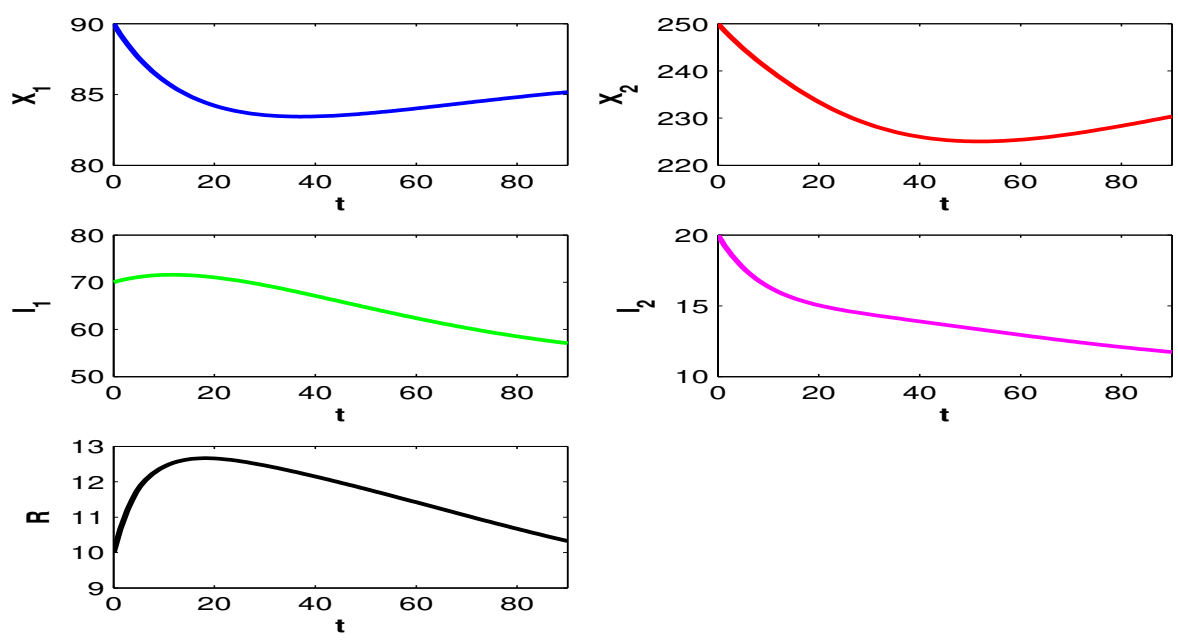

Fig. 16. Profiles of populations with applied optimal treatment $u_{2}^{*}$ only and $u_{1}=u_{3}=0$.
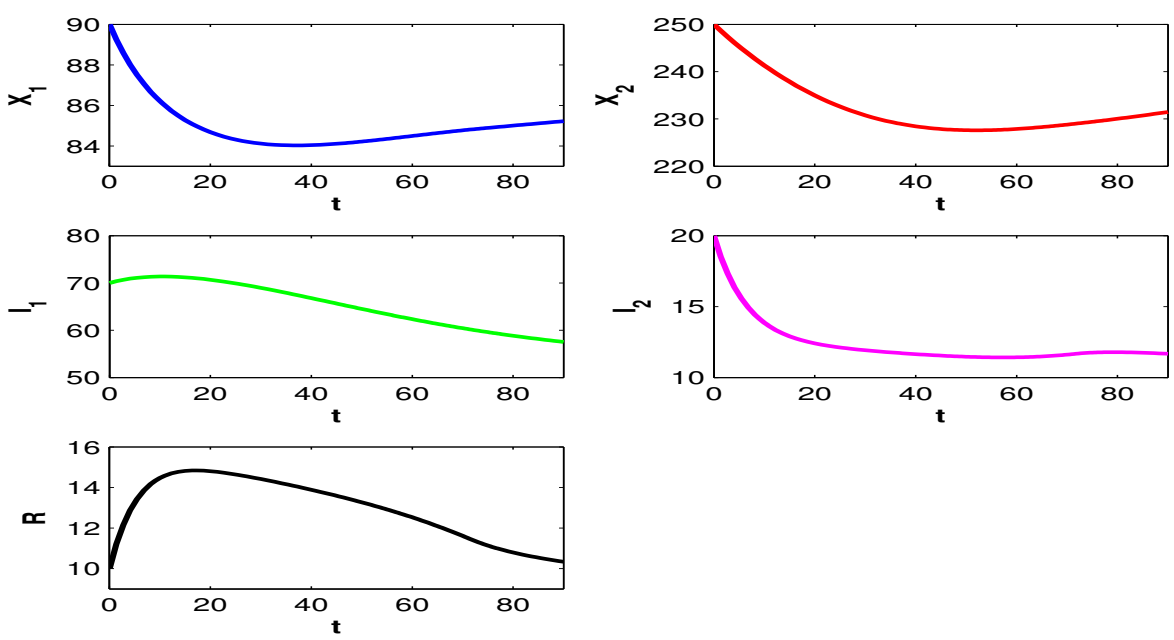

Fig. 17. Profiles of populations with applied optimal treatment $u_{3}^{*}$ only and $u_{1}=u_{2}=0$.

Trajectory profiles in Figure 17 depict the population when the treatment $\left(u_{3}\right)$ applied to symptomatically infected population is considered. At $T_{f}=90$, the population is (85.22158663, 231.45762501, 57.53470914, 11.67773883, 10.33995505). Providing better treatment to the symptomatically infected population can control the disease transmission significantly as this also increases both susceptible and stressed population and also recovered population. Moreover, the symptomatically infected population also decreases. The bottom figure of left column in Figure 18 represents the path of optimal intensity denoting the pharmaceutical treatment provided to the symptomatically infected population. This control policy works with higher intensity in the early stages and then it decreases with time. The treatment policy works for quite a long time and can control the disease burden with higher intensity.

Trajectory profiles in Figure 19 depict the population when vaccination applied to both susceptible and stressed population. At $T_{f}=90$, the population is (85.16818672, 230.34242204, $57.07635678,11.73587788,10.32661249)$. Providing immunization also increases the number of individuals in the susceptible and stressed population. So, a lesser number of people get infected and the disease can not transmit that much. 

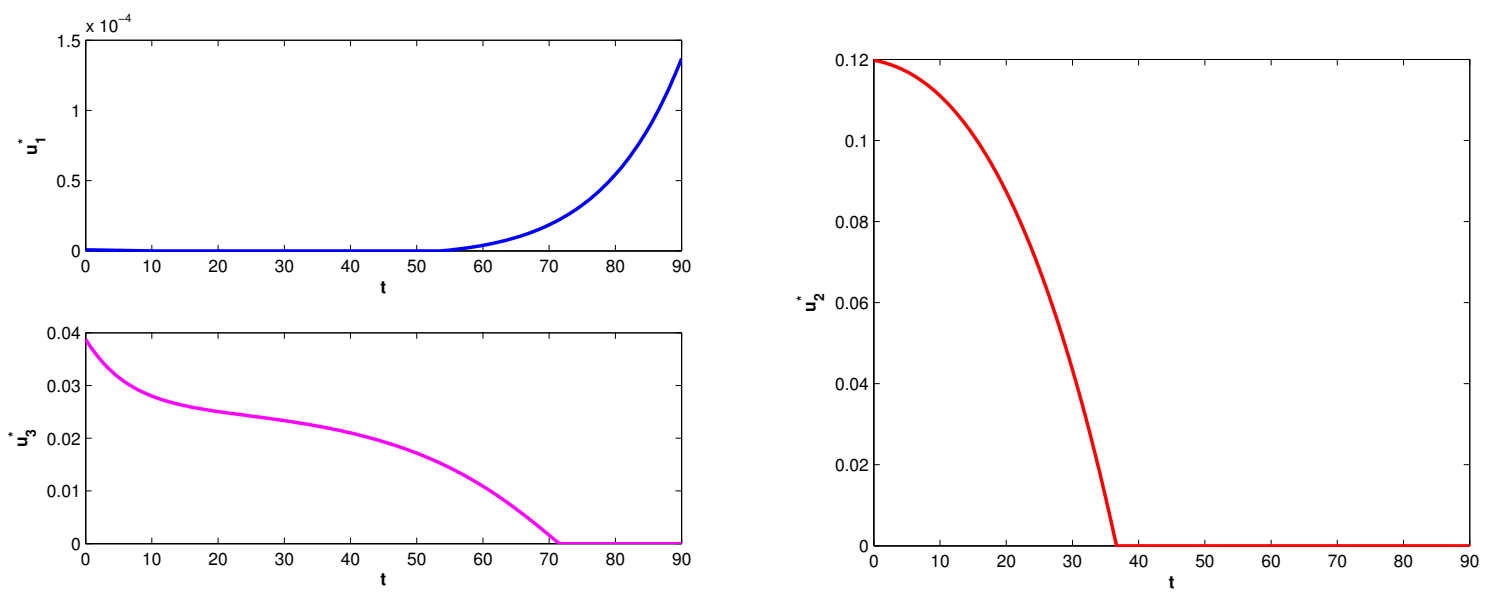

Fig. 18. Optimal intensity of treatment $u_{1}^{*}$ when $u_{2}=u_{3}=0$; $u_{2}^{*}$ when $u_{1}=u_{3}=0$ and $u_{3}^{*}$ when $u_{2}=u_{3}=0$.
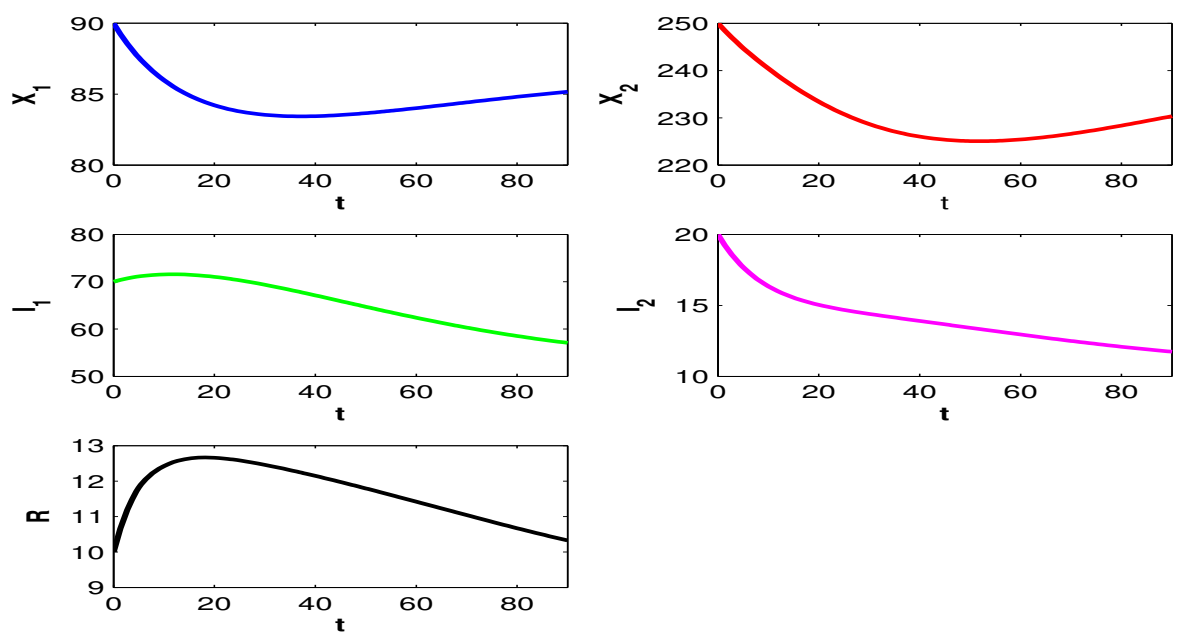

Fig. 19. Profiles of populations with applied optimal treatments $u_{1}^{*}$ and $u_{2}^{*}$ but $u_{3}=0$.

Trajectory profiles in Figure 20 depict the population when the susceptible population takes vaccine as a precaution as well as the symptomatically infected population goes through some better treatment policies. At $T_{f}=90$, the population is (85.22158566, 231.45762497, 57.53470902, 11.67773882, 10.33995621). Providing vaccine to susceptible individuals and treatment implemented to symptomatically infected population lead to more individuals in susceptible as well as recovered class. The size of symptomatically infected population decreases with a higher rate than the case when no control policies are applied.

Trajectory profiles in Figure 21 depict the population size when the stressed population takes vaccine as a precaution as well as the symptomatically infected population goes through some effective treatment policies. At $T_{f}=90$, the population size is (85.22485747, 231.49232666, $57.53314564,11.67620633,10.33877987)$. So, effective treatment on symptomatically infected population controls the disease transmission resulting in an increase of susceptible and stressed population and also recovered population. And the symptomatically infected population also decreases in this situation.

Implementation of the control policies is beneficial for the underlying system. Next, let us consider the combination of three control policies simultaneously, i.e., a system where 

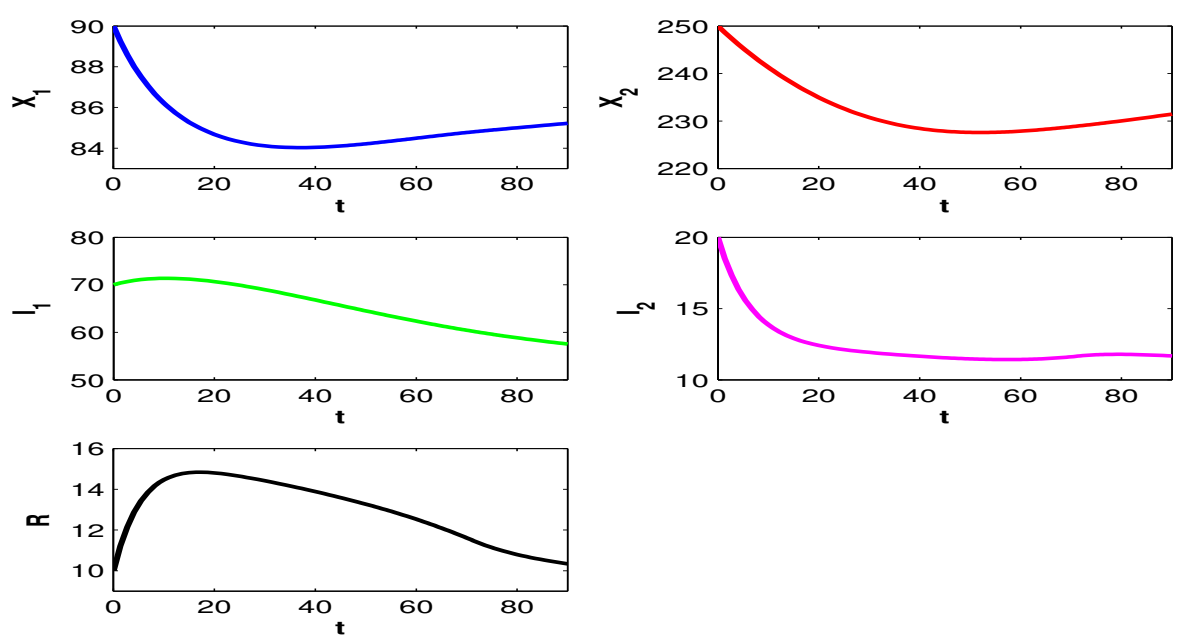

Fig. 20. Profiles of populations with applied optimal treatments $u_{1}^{*}$ and $u_{3}^{*}$ but $u_{2}=0$.
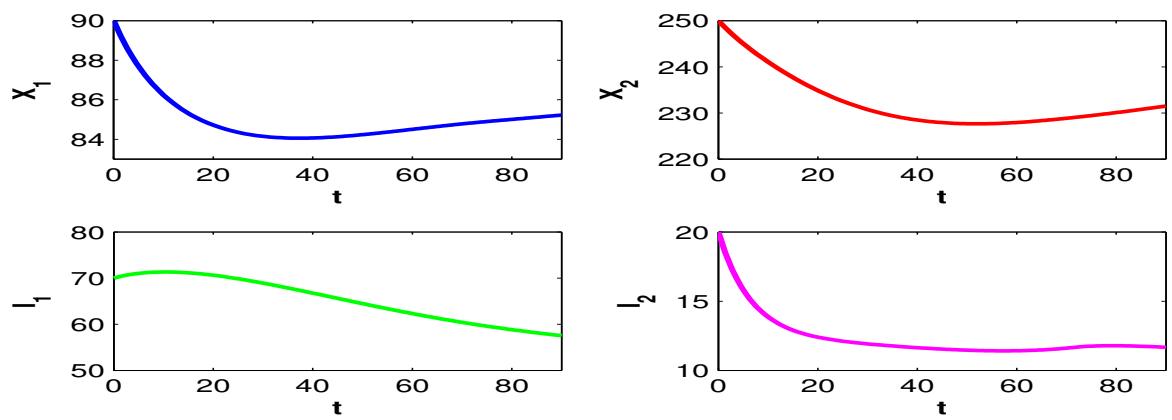

n

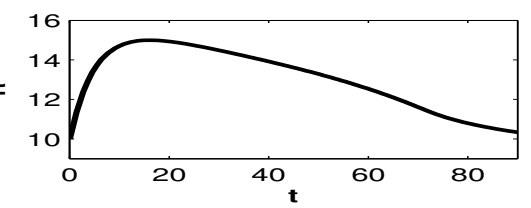

Fig. 21. Profiles of populations with applied optimal treatments $u_{2}^{*}$ and $u_{3}^{*}$ but $u_{1}=0$.
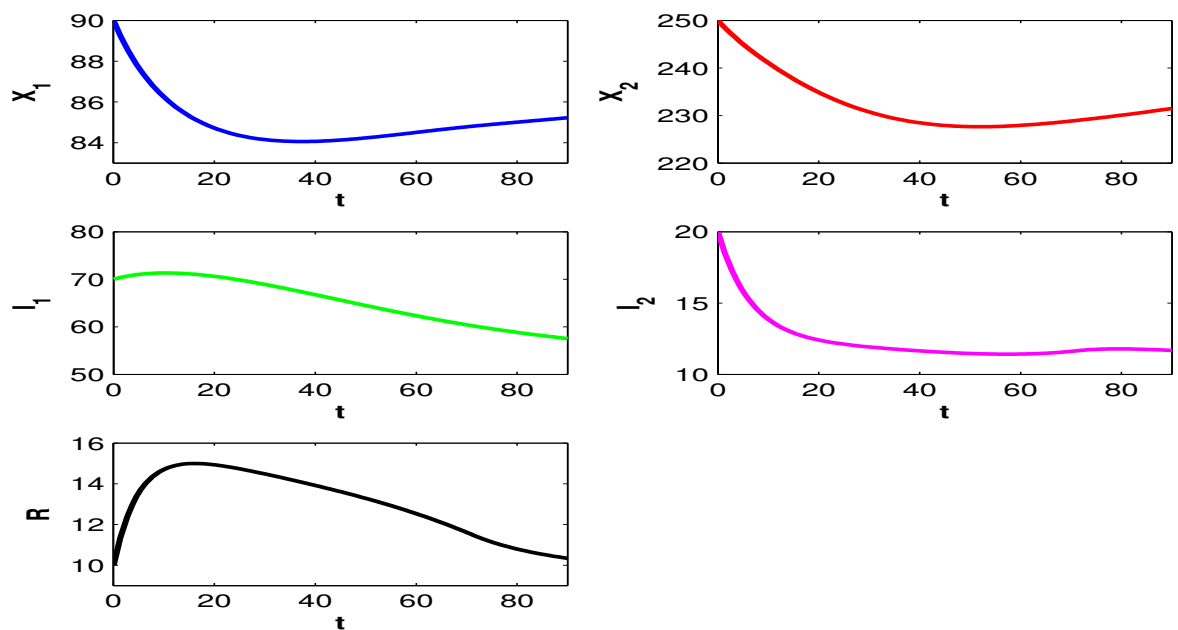

Fig. 22. Profiles of populations with both optimal control policies $u_{1}^{*}, u_{2}^{*}$ and $u_{3}^{*}$. Parameters are as in Table 2. 
the susceptible and stressed population are under vaccination strategy and effective treatment policy is applied to symptomatically infected individuals. Figure $\underline{22}$ depicts the trajectory profiles of the population in the presence of all control interventions. Here at $T_{f}=90$, the population size is (85.22485651, 231.49232661, 57.53314535, 11.67620632, 10.33878102). So, the implementation of all the control policies works better to control disease transmission as expected. Vaccination and treatment policies reduce the overall infected population and also increase the susceptible population. The paths of the optimal control strategies have been depicted in Figure 23. As per the simulations performed in this work: (i) the control policy representing vaccination applied to susceptible individuals works better in the later stage, i.e., it works after almost two months; (ii) the control policy for vaccination applied to stressed individuals works better in the earlier stage, i.e., it works better for almost first month and (iii) the control policy regarding treatment applied to symptomatically infected individuals works with higher intensity in the earlier stage but it decreases with time.
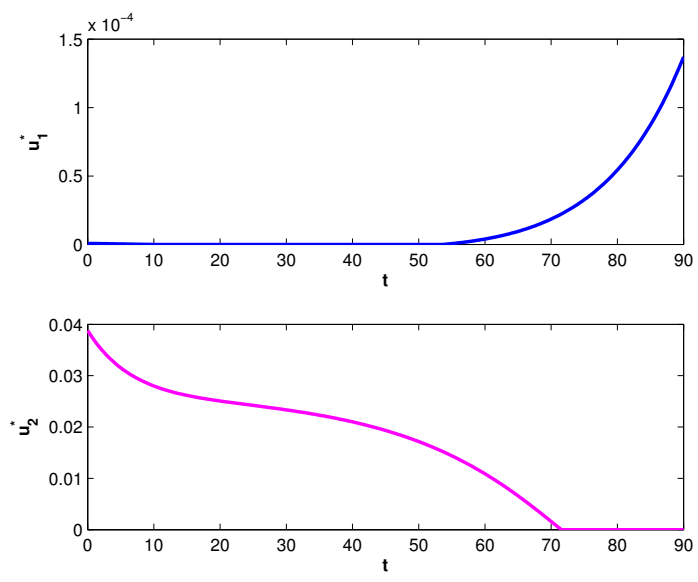

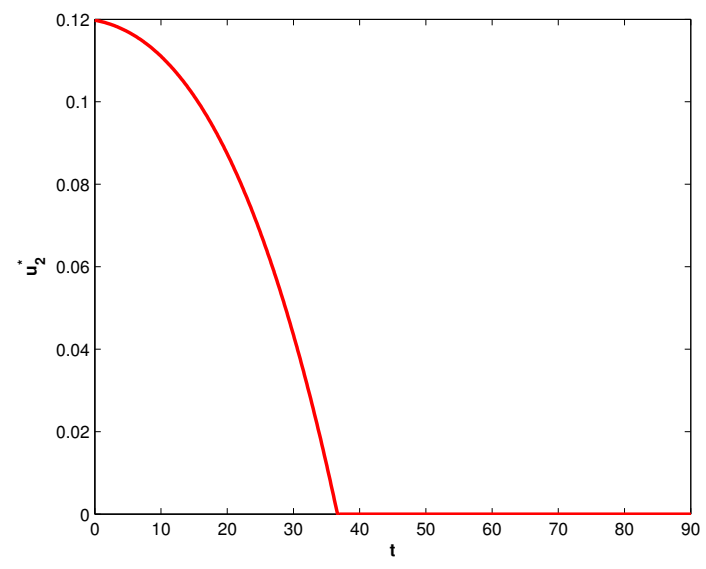

Fig. 23. Profiles of optimal controls $u_{1}^{*}, u_{2}^{*}$ and $u_{3}^{*}$. Other parameters are as in Table 2 .

The cost design analysis in presence as well as in absence of controls have been performed in Figure 24 because cost-effectiveness is one of the major attributes to determine the fitness. Two cases have been considered here: (i) when there is no implemented control policy and (ii) when all the control policies are implemented in the corresponding system. Optimal cost profiles for both the cases are depicted in Figure 24,a. Moreover, the trajectory profiles of symptomatically infected individuals and recovered individuals can be observed from figures 24,b and 24,c respectively. The cost, in absence of any control policy, occurs because of productivity loss by both infective populations. The opportunity loss is higher as the disease spread out and both the infected population increase with higher rates. As a result, after a certain time economic burden also increases. On the other hand, in the presence of all control policies in the system, the optimal cost is lower than the case when no control policy is implemented. Lesser number of infected individuals mainly reduce the overall cost due to opportunity loss.

\subsection{Effect of Saturated Treatment on Optimal Control Policies}

This section contains how pharmaceutical treatment affects the disease dynamics in the presence of control policies with optimal intensities. Saturation rate on treatment $\left(\zeta_{3}^{-1}\right)$ and the treatment rate $\left(\epsilon_{3}\right)$ have been varied in the following figures. Figure 25 shows the graphs of symptomatically infected population and corresponding cost for different values of $\zeta_{3}$. With the increase of $\zeta_{3}$, the number of infected individuals, as well as the associated cost, also increase because of the proliferation of infective related productivity loss. The optimal controls have been 


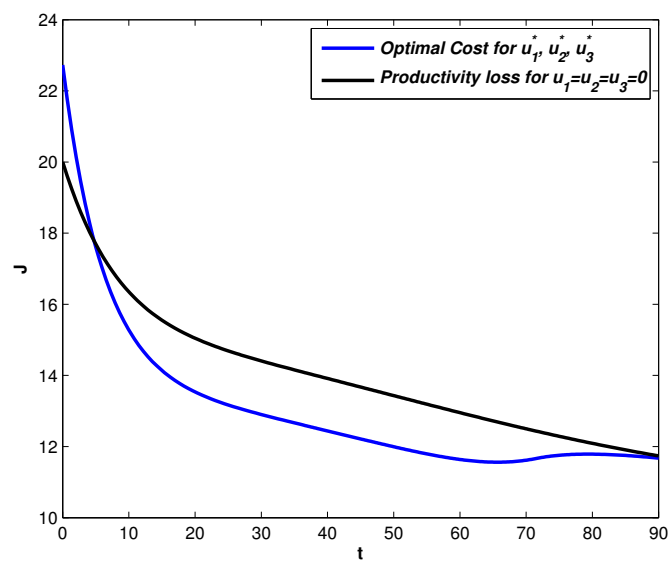

(a)

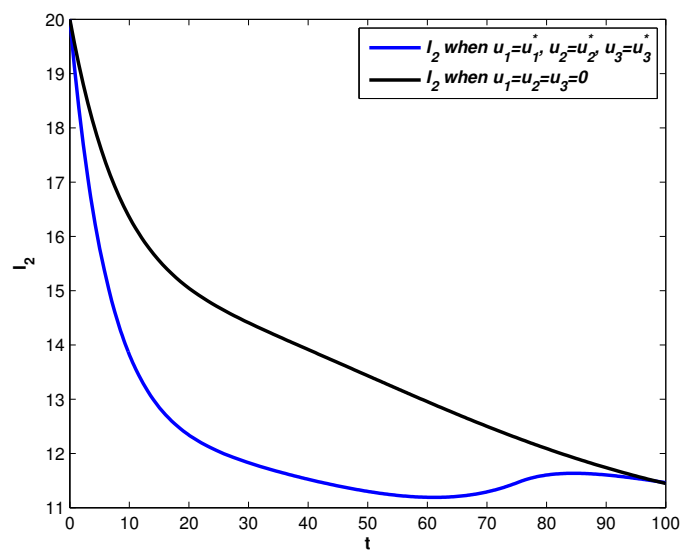

(b)

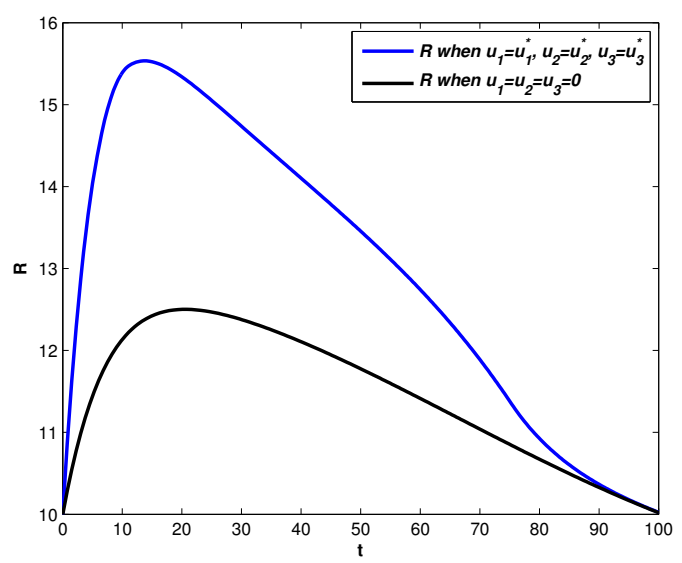

(c)

Fig. 24. (a) Cost distribution in presence and absence of control policies. (b) Profiles of symptomatically infected population under different control policies. (c) Profiles of recovered population in presence and absence of control policies. Other parameters are as in Table 2.

drawn in Figure 26 from which it is observed that for increasing value of $\zeta_{3}$, the control policy denoting vaccination for susceptible individuals starts to show its impact earlier. It means that smaller saturation rate increases the time period during which the control policy is implemented successfully. Also, increasing value of $\zeta_{3}$ increases the intensity level of the control denoting vaccination for the stressed population too. On the contrary, increasing value of $\zeta_{3}$ decreases the intensity level of the control denoting treatment of the symptomatically infected population.

Further, when the treatment rate $\left(\epsilon_{3}\right)$ is varied from 0.5 to 0.8 , the symptomatically infected population decreases with time following a reduction in associated cost too (Fig. 27). From Figure 28, it is observed that for increasing value of $\epsilon_{3}$, the control policy denoting vaccination for susceptible individuals starts to show its impact later. It means that higher treatment rate decreases the time period during which the control policy (for vaccination) is implemented successfully. Also, increasing value of $\epsilon_{3}$ decreases the intensity level of the control denoting vaccination for the stressed population too. On the contrary, increasing value of $\epsilon_{3}$ increases the intensity level of the control denoting treatment of the symptomatically infected population. 


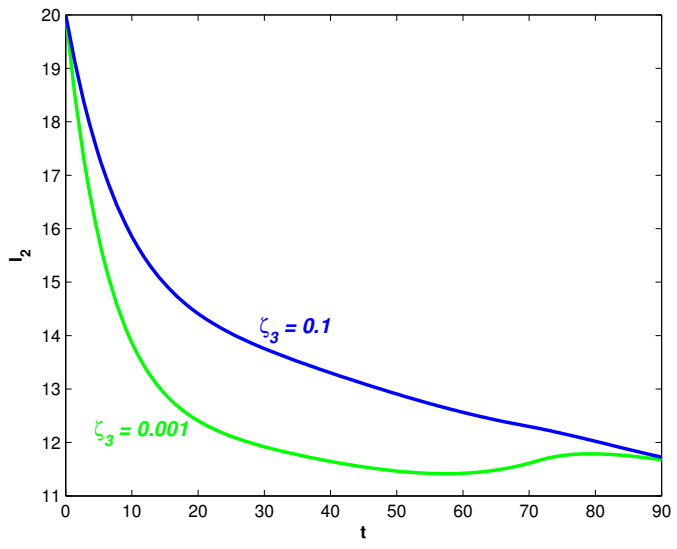

(a)

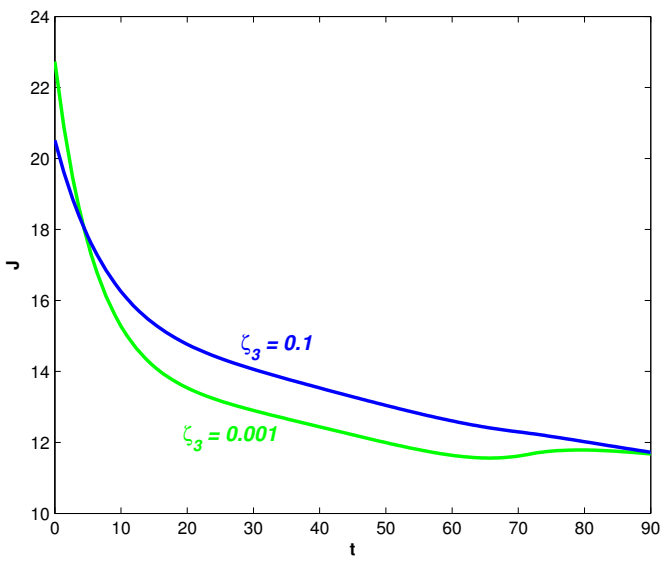

(b)

Fig. 25. (a) Profiles of symptomatically infected population for various $\zeta_{3}$ with $u_{1}^{*}, u_{2}^{*}$ and $u_{3}^{*}$. (b) Profiles of cost for various $\zeta_{3}$ with $u_{1}^{*}, u_{2}^{*}$ and $u_{3}^{*}$. Other parameters are as in Table 2.

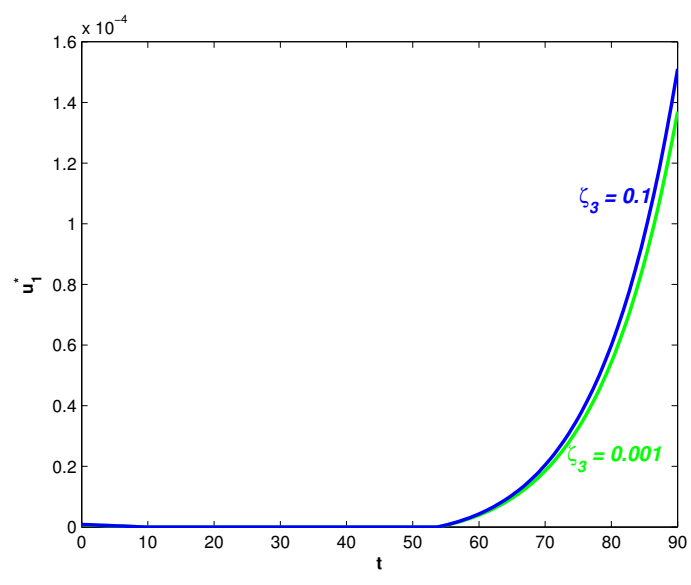

(a)

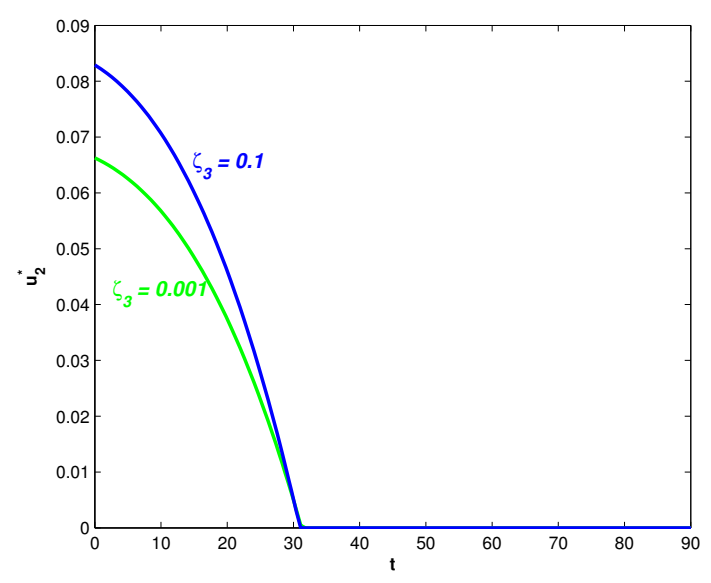

(b)

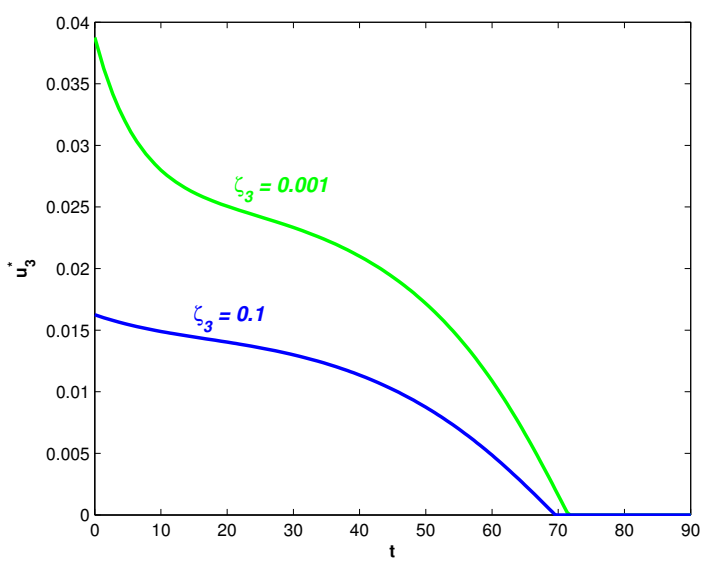

(c)

Fig. 26. (a) Plots of control $u_{1}^{*}$ for various $\zeta_{3}$. (b) Plots of control $u_{2}^{*}$ for various $\zeta_{3}$. (c) Plots of control $u_{3}^{*}$ for various $\zeta_{3}$. Other parameters are as in Table 2 . 


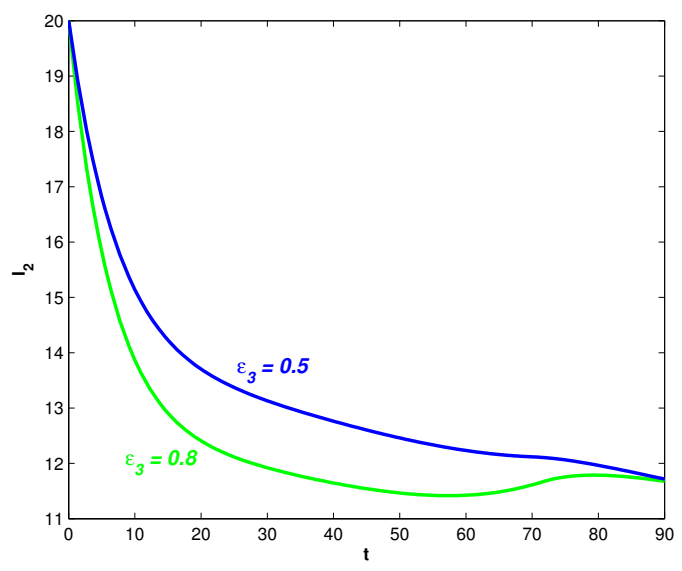

(a)

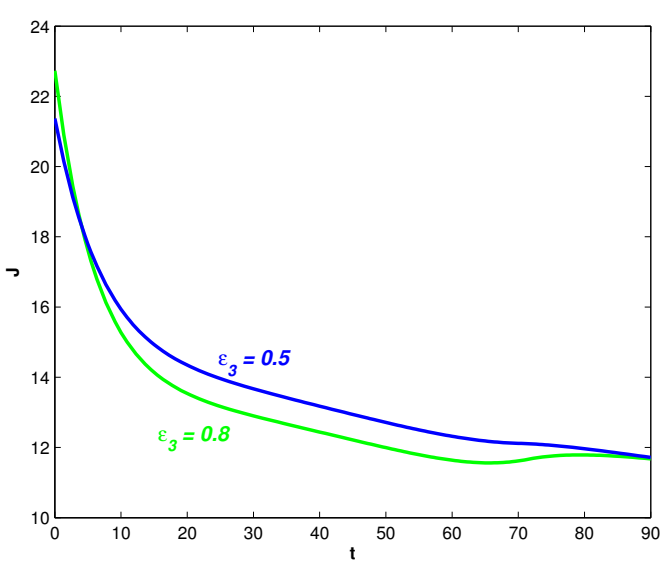

(b)

Fig. 27. (a) Profiles of infected population for various $\epsilon_{3}$ with $u_{1}^{*}, u_{2}^{*}$ and $u_{3}^{*}$. (b) Profiles of cost for various $\epsilon$ with $u_{1}^{*}, u_{2}^{*}$ and $u_{3}^{*}$. Other parameters are as in Table 2 .

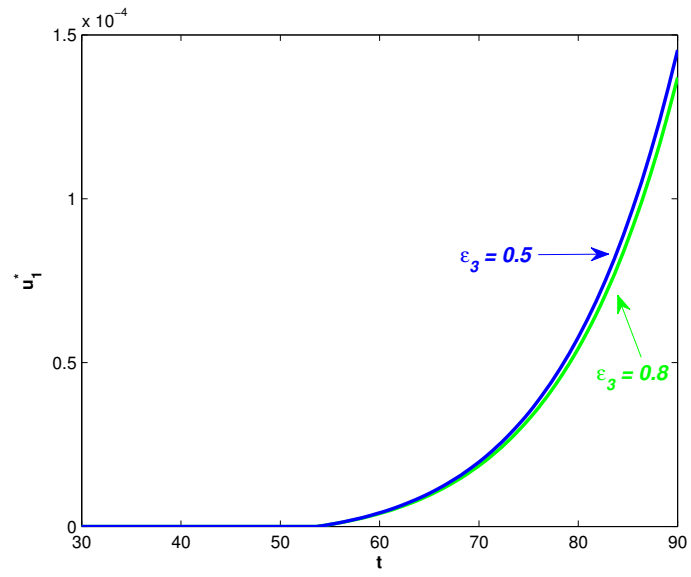

(a)

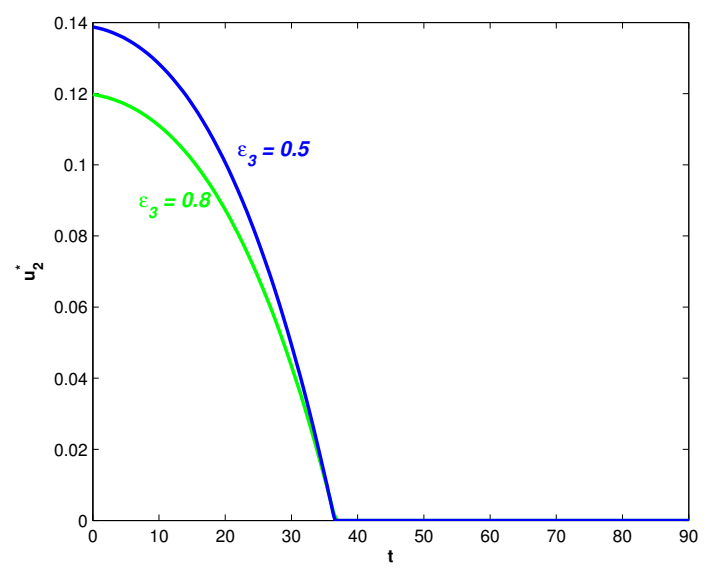

(b)

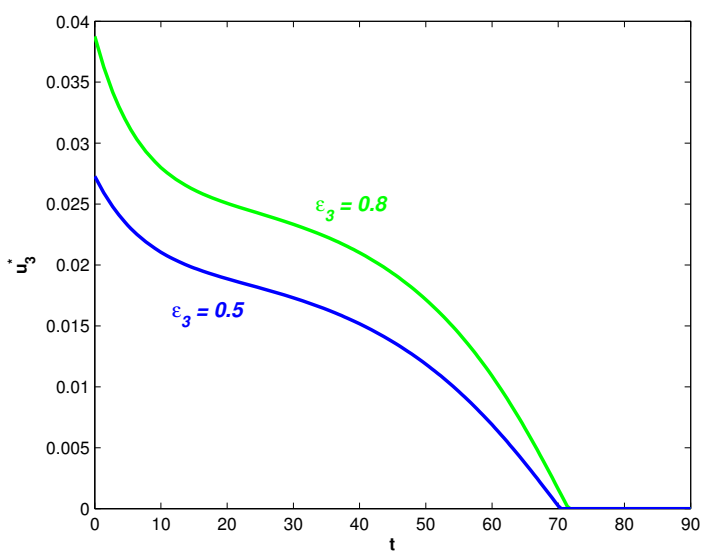

(c)

Fig. 28. (a) Plots of control $u_{1}^{*}$ for various $\epsilon_{3}$. (b) Plots of control $u_{2}^{*}$ for various $\epsilon_{3}$. (c) Plots of control $u_{3}^{*}$ for various $\epsilon_{3}$. Other parameters are as in Table 2 . 


\section{CONCLUSION}

Environmental stress mainly expedite the effect of pollutants on organisms. In this work, a compartmental model with infectious disease has been proposed in system (1) to analyse the impact of environmental stress on the dynamics of disease transmission. Pictorial scenarios have been depicted to show how environmental stress affects disease transmission. For increasing values of $\theta$ and $p$, the asymptomatically infected population also increases. It means, if the stressed population increases, it will lead to the inclination of the infected population also. Also, asymptomatically infected population with respect to the stress parameters $\omega_{1}, \omega_{2}$ and $\beta_{1}^{\prime}, \beta_{2}^{\prime}$ increases for increasing values of $p$ and $\theta$. But the increment of infected individuals are higher for $\omega_{1}, \beta_{1}^{\prime}$ rather than $\omega_{2}, \beta_{2}^{\prime}$. Now the basic reproduction number $R_{0}$ also increases for increasing environmental stress level but it is shown that $R_{0}$ is most sensitive to the disease transmission rate among asymptomatically infected individuals $\left(\beta_{1}\right)$. As the basic reproduction number denotes the average number of newly infective from a single infected, then it is evident that increasing the value of $R_{0}$ causes more infected individuals in the system.

Introducing optimal control policies reduce the disease prevalence to some extent. From the numerical scenarios of Section 10 it is observed that, if we apply any one of the control policies, that should be effective but with certain restrictions. Optimal response due to the vaccination policy among the individuals of the susceptible population works better at a later stage while vaccination policy for stressed people works with higher intensity at an earlier stage of the infected period. With time the vaccination control intensity (among individuals of the stressed population) decreases and ultimately it does not make any impact to reduce the disease burden. So, providing a suitable vaccine to the susceptible population at the beginning can reduce the economic burden. Moreover, the control policy corresponding to effective treatment for symptomatic individuals works better in the first month of infection and then starts to decrease (as per the simulations). Also, the optimal treatment can minimize the cost burden as well as the size of overall infected individuals and the duration of disease prevalence. It is evident that the overall infected population decreases significantly in the presence of all the control interventions rather than in the absence of control policies. These policies can minimize the overall economic load also. So, implementation of all control policies is more effective and economical to reduce disease transmission in the presence of environmental pollution.

The first author (Sangeeta Saha) is thankful to the University Grants Commission, India for providing SRF. 


\section{APPENDIX}

\section{Existence of Optimal Control Functions}

Here we derive the conditions for which optimal control interventions exist and minimize the cost function within a finite time.

\section{Proof of Theorem 10}

P r o o f. For existence of optimal controls, the following conditions need to be satisfied:

(i) Set of solutions of system (7) with control variables $u_{1}, u_{2}$ and $u_{3}$ in $\Pi \neq \phi$.

(ii) $\Pi$ is closed, convex and state system can be expressed as a linear function of control variables with coefficients depending on time and state variables.

(iii) The integrand of (6) denoted as $L$ is convex on $\Pi$ and $L\left(X_{1}, X_{2}, I_{1}, I_{2}, R, u_{1}, u_{2}, u_{3}\right) \geq$ $g\left(u_{1}, u_{2}, u_{3}\right)$ where $g\left(u_{1}, u_{2}, u_{3}\right)$ is continuous and $\left\|\left(u_{1}, u_{2}, u_{3}\right)\right\|^{-1} g\left(u_{1}, u_{2}, u_{3}\right) \rightarrow \infty$ whenever $\left\|\left(u_{1}, u_{2}, u_{3}\right)\right\| \rightarrow \infty$; $\|$.$\| represents the L^{3}\left(0, T_{f}\right)$ norm.

From (7), the total population $N=X_{1}+X_{2}+I_{1}+I_{2}+R$.

$$
\text { So, } \begin{aligned}
\frac{d N}{d t} & =\Lambda-d N-\delta\left(I_{1}+I_{2}\right) \leq \Lambda-d N \\
\Rightarrow 0 & <N(t) \leq N(0) e^{-d t}+\frac{\Lambda}{d}\left(1-e^{-d t}\right),
\end{aligned}
$$

where $N(0)$ represents the sum of the initial values of the variables $\left(X_{1}, X_{2}, I_{1}, I_{2}, R\right)$. As $t \rightarrow \infty, 0<N(t) \leq \frac{\Lambda}{d}$.

For each control variable in $\Pi$, the solution of (7) is bounded as well as right hand side functions are locally Lipschitzian with respect to all the variables. So, by Picard - Lindelöf theorem, condition (i) is satisfied [61].

Now the control set $\Pi$ is closed and convex by definition. Again each equation of system (7) can be written as a linear equation in control variables $u_{1}, u_{2}$ and $u_{3}$ with state variables dependent coefficients and so condition (ii) is also satisfied. Now the quadratic nature of all control variables $u_{1}, u_{2}$ and $u_{3}$ ensure that the integrand $L\left(X_{1}, X_{2}, I_{1}, I_{2}, R, u_{1}, u_{2}, u_{3}\right)$ is convex.

$$
\text { Also, } \begin{aligned}
L\left(X_{1}, X_{2}, I_{1}, I_{2}, R, u_{1}, u_{2}, u_{3}\right) & =w_{1} I_{2}+w_{2} u_{1}^{2}+w_{3} u_{2}^{2}+w_{4} u_{3}^{2} \\
& \geq w_{2} u_{1}^{2}+w_{3} u_{2}^{2}+w_{4} u_{3}^{2}
\end{aligned}
$$

Let, $\bar{c}=\min \left(w_{2}, w_{3}, w_{4}\right)>0$ and $g\left(u_{1}, u_{2}, u_{3}\right)=\bar{c}\left(u_{1}^{2}+u_{2}^{2}+u_{3}^{2}\right)$.

Then $L\left(X_{1}, X_{2}, I_{1}, I_{2}, R, u_{1}, u_{2}, u_{3}\right) \geq g\left(u_{1}, u_{2}, u_{3}\right)$.

Here $g$ is continuous and $\left\|\left(u_{1}, u_{2}, u_{3}\right)\right\|^{-1} g\left(u_{1}, u_{2}, u_{3}\right) \rightarrow \infty$ whenever $\left\|\left(u_{1}, u_{2}, u_{3}\right)\right\| \rightarrow \infty$. Hence, condition (iii) holds good. From $[\underline{62}, \underline{50}]$, it is concluded that there exists control interventions $u_{1}^{*}, u_{2}^{*}$ and $u_{3}^{*}$ such that $J\left[u_{1}^{*}, u_{2}^{*}, u_{3}^{*}\right]=\min \left[J\left[u_{1}, u_{2}, u_{3}\right]\right]$.

\section{Characterization of Optimal Control Functions}

By Pontryagin's Maximum Principle, we have derived here the necessary conditions for optimal control functions for system (6)-(7) [62, 63]. Let us define the Hamiltonian function as:

$$
\begin{aligned}
H\left(X_{1}, X_{2}, I_{1}, I_{2}, R, u_{1}, u_{2}, u_{3}, \Psi\right) & =L\left(X_{1}, X_{2}, I_{1}, I_{2}, R, u_{1}, u_{2}, u_{3}\right)+\lambda_{1} \frac{d X_{1}}{d t}+\lambda_{2} \frac{d X_{2}}{d t}+\lambda_{3} \frac{d I_{1}}{d t} \\
& +\lambda_{4} \frac{d I_{2}}{d t}+\lambda_{5} \frac{d R}{d t} \\
237 &
\end{aligned}
$$


So, $H=w_{1} I_{2}+w_{2} u_{1}^{2}+w_{3} u_{2}^{2}+w_{4} u_{3}^{2}+\lambda_{1}\left[(1-p) \Lambda-d X_{1}-\theta X_{1}+(1-q) \gamma R\right.$

$$
\begin{aligned}
& \left.-\left(\beta_{1} I_{1}+\beta_{2} I_{2}\right) X_{1}-\frac{\epsilon_{1} u_{1} X_{1}}{1+\zeta_{1} X_{1}}\right]+\lambda_{2}\left[p \Lambda+\theta X_{1}-d X_{2}+q \gamma R\right. \\
& \left.-\left\{\beta_{1}\left(1+\omega_{1} \beta_{1}^{\prime}\right) I_{1}+\beta_{2}\left(1+\omega_{2} \beta_{2}^{\prime}\right) I_{2}\right\} X_{2}-\frac{\epsilon_{2} u_{2} X_{2}}{1+\zeta_{2} X_{2}}\right]+\lambda_{3}\left[\left(\beta_{1} I_{1}+\beta_{2} I_{2}\right) X_{1}\right. \\
& \left.+\left\{\beta_{1}\left(1+\omega_{1} \beta_{1}^{\prime}\right) I_{1}+\beta_{2}\left(1+\omega_{2} \beta_{2}^{\prime}\right) I_{2}\right\} X_{2}-(d+\delta+\eta) I_{1}\right]+\lambda_{4}\left[\eta I_{1}-(d+\delta) I_{2}-\alpha I_{2}\right. \\
& \left.-\frac{\epsilon_{3} u_{3} I_{2}}{1+\zeta_{3} I_{2}}\right]+\lambda_{5}\left[\alpha I_{2}-(\gamma+d) R+\frac{\epsilon_{1} u_{1} X_{1}}{1+\zeta_{1} X_{1}}+\frac{\epsilon_{2} u_{2} X_{2}}{1+\zeta_{2} X_{2}}+\frac{\epsilon_{3} u_{3} I_{2}}{1+\zeta_{3} I_{2}}\right]
\end{aligned}
$$

Here $\Psi=\left(\lambda_{1}, \lambda_{2}, \lambda_{3}, \lambda_{4}, \lambda_{5}\right)$ is the adjoint variable. We get minimized Hamiltonian by Pontryagin's Maximum Principle to minimize the cost functional. Pontryagin's Maximum Principle mainly adjoin the cost functional with the state equations by introducing adjoint variables.

\section{Proof of Theorem 11}

Proof. Let $u_{1}^{*}, u_{2}^{*}$ and $u_{3}^{*}$ be optimal control variables and $X_{1}^{*}, X_{2}^{*}, I_{1}^{*}, I_{2}^{*}, R^{*}$ are corresponding optimal state variables of the control system (7) which minimize the cost functional (6). So, by Pontryagin's Maximum Principle, there exist adjoint variables $\lambda_{1}, \lambda_{2}, \lambda_{3}, \lambda_{4}, \lambda_{5}$ which satisfy the following canonical equations:

$$
\frac{d \lambda_{1}}{d t}=-\frac{\partial H}{\partial X_{1}}, \quad \frac{d \lambda_{2}}{d t}=-\frac{\partial H}{\partial X_{2}}, \quad \frac{d \lambda_{3}}{d t}=-\frac{\partial H}{\partial I_{1}}, \quad \frac{d \lambda_{4}}{d t}=-\frac{\partial H}{\partial I_{2}}, \quad \frac{d \lambda_{4}}{d t}=-\frac{\partial H}{\partial R} .
$$

So, we have

$$
\begin{aligned}
\frac{d \lambda_{1}}{d t} & =\lambda_{1}\left[d+\theta+\beta_{1} I_{1}+\beta_{2} I_{2}+\frac{\epsilon_{1} u_{1}(t)}{\left(1+\zeta_{1} X_{1}\right)^{2}}\right]-\lambda_{2} \theta-\lambda_{3}\left(\beta_{1} I_{1}+\beta_{2} I_{2}\right)-\lambda_{5} \frac{\epsilon_{1} u_{1}(t)}{\left(1+\zeta_{1} X_{1}\right)^{2}} \\
\frac{d \lambda_{2}}{d t} & =\lambda_{2}\left[d+\beta_{1}\left(1+\omega_{1} \beta_{1}^{\prime}\right) I_{1}+\beta_{2}\left(1+\omega_{2} \beta_{2}^{\prime}\right) I_{2}+\frac{\epsilon_{2} u_{2}}{\left(1+\zeta_{2} X_{2}\right)^{2}}\right] \\
& -\lambda_{3}\left[\beta_{1}\left(1+\omega_{1} \beta_{1}^{\prime}\right) I_{1}+\beta_{2}\left(1+\omega_{2} \beta_{2}^{\prime}\right) I_{2}\right]-\lambda_{5} \frac{\epsilon_{2} u_{2}(t)}{\left(1+\zeta_{2} X_{2}\right)^{2}}, \\
\frac{d \lambda_{3}}{d t} & =\lambda_{1} \beta_{1} X_{1}+\lambda_{2} \beta_{1}\left(1+\omega_{1} \beta_{1}^{\prime}\right) X_{2}-\lambda_{3}\left\{\beta_{1} X_{1}+\beta_{1}\left(1+\omega_{1} \beta_{1}^{\prime}\right) X_{2}-(d+\delta+\eta)\right\}-\lambda_{4} \eta \\
\frac{d \lambda_{4}}{d t} & =-w_{1}+\lambda_{1} \beta_{2} X_{1}+\lambda_{2} \beta_{2}\left(1+\omega_{2} \beta_{2}^{\prime}\right) X_{2}-\lambda_{3}\left\{\beta_{2} X_{1}+\beta_{2}\left(1+\omega_{2} \beta_{2}^{\prime}\right) X_{2}\right\} \\
& +\lambda_{4}\left(d+\delta+\alpha+\frac{\epsilon_{3} u_{3}(t)}{\left(1+\zeta_{3} I_{2}\right)^{2}}\right)-\lambda_{5}\left(\alpha+\frac{\epsilon_{3} u_{3}(t)}{\left(1+\zeta_{3} I_{2}\right)^{2}}\right) \\
\frac{d \lambda_{5}}{d t} & =-\lambda_{1}(1-q) \gamma-\lambda_{2} q \gamma+\lambda_{5}(d+\gamma)
\end{aligned}
$$

with the transversality conditions $\lambda_{i}\left(T_{f}\right)=0$, for $i=1,2,3,4,5$.

$$
\text { From optimality conditions : }\left.\frac{\partial H}{\partial u_{1}}\right|_{u_{1}=u_{1}^{*}}=0,\left.\frac{\partial H}{\partial u_{2}}\right|_{u_{2}=u_{2}^{*}}=0 \text { and }\left.\frac{\partial H}{\partial u_{3}}\right|_{u_{3}=u_{3}^{*}}=0 \text {. }
$$

So, $u_{1}^{*}=\frac{\epsilon_{1} X_{1}^{*}}{2 w_{2}\left(1+\zeta_{1} X_{1}^{*}\right)}\left(\lambda_{1}-\lambda_{5}\right), u_{2}^{*}=\frac{\epsilon_{2} X_{2}^{*}}{2 w_{3}\left(1+\zeta_{2} X_{2}^{*}\right)}\left(\lambda_{2}-\lambda_{5}\right)$ and $u_{3}^{*}=\frac{\epsilon_{3} I_{2}^{*}}{2 w_{4}\left(1+\zeta_{3} I_{2}^{*}\right)}\left(\lambda_{4}-\lambda_{5}\right)$. Now 
from these findings along with the characteristics of control set $\Pi$, we have

$$
\begin{aligned}
& u_{1}^{*}= \begin{cases}0, & \text { if } \frac{\epsilon_{1} X_{1}^{*}}{2 w_{2}\left(1+\zeta_{1} X_{1}^{*}\right)}\left(\lambda_{1}-\lambda_{5}\right)<0 \\
\frac{\epsilon_{1} X_{1}^{*}}{2 w_{2}\left(1+\zeta_{1} X_{1}^{*}\right)}\left(\lambda_{1}-\lambda_{5}\right), & \text { if } 0 \leq \frac{\epsilon_{1} X_{1}^{*}}{2 w_{2}\left(1+\zeta_{1} X_{1}^{*}\right)}\left(\lambda_{1}-\lambda_{5}\right) \leq 1 \\
1, & \text { if } \frac{\epsilon_{1} X_{1}^{*}}{2 w_{2}\left(1+\zeta_{1} X_{1}^{*}\right)}\left(\lambda_{1}-\lambda_{5}\right)>1\end{cases} \\
& u_{2}^{*}= \begin{cases}0, & \text { if } \frac{\epsilon_{2} X_{2}^{*}}{2 w_{3}\left(1+\zeta_{2} X_{2}^{*}\right)}\left(\lambda_{2}-\lambda_{5}\right)<0 \\
\frac{\epsilon_{2} X_{2}^{*}}{2 w_{3}\left(1+\zeta_{2} X_{2}^{*}\right)}\left(\lambda_{2}-\lambda_{5}\right), & \text { if } 0 \leq \frac{\epsilon_{2} X_{2}^{*}}{2 w_{3}\left(1+\zeta_{2} X_{2}^{*}\right)}\left(\lambda_{2}-\lambda_{5}\right) \leq 1 \\
1, & \text { if } \frac{\epsilon_{2} X_{2}^{*}}{2 w_{3}\left(1+\zeta_{2} X_{2}^{*}\right)}\left(\lambda_{2}-\lambda_{5}\right)>1\end{cases} \\
& u_{3}^{*}= \begin{cases}0, & \text { if } \frac{\epsilon_{3} I_{2}^{*}}{2 w_{4}\left(1+\zeta_{3} I_{2}^{*}\right)}\left(\lambda_{4}-\lambda_{5}\right)<0 \\
\frac{\epsilon_{3} I_{2}^{*}}{2 w_{4}\left(1+\zeta_{3} I_{2}^{*}\right)}\left(\lambda_{4}-\lambda_{5}\right), & \text { if } 0 \leq \frac{\epsilon_{3} I_{2}^{*}}{2 w_{4}\left(1+\zeta_{3} I_{2}^{*}\right)}\left(\lambda_{4}-\lambda_{5}\right) \leq 1 \\
1, & \text { if } \frac{\epsilon_{3} I_{2}^{*}}{2 w_{4}\left(1+\zeta_{3} I_{2}^{*}\right)}\left(\lambda_{4}-\lambda_{5}\right)>1\end{cases}
\end{aligned}
$$

which is equivalent as (9).

\section{Optimal System}

Now we state the optimal system with the optimal control variables $u_{1}^{*}, u_{2}^{*}$ and $u_{3}^{*}$ defined in section 9. The optimal system with minimized Hamiltonian $H^{*}$ at $\left(X_{1}^{*}, X_{2}^{*}, I_{1}^{*}, I_{2}^{*}, R^{*}, \lambda_{1}\right.$, $\left.\lambda_{2}, \lambda_{3}, \lambda_{4}, \lambda_{5}\right)$ is as follows:

$$
\begin{aligned}
\frac{d X_{1}^{*}}{d t} & =(1-p) \Lambda-d X_{1}^{*}-\theta X_{1}^{*}+(1-q) \gamma R^{*}-\left(\beta_{1} I_{1}^{*}+\beta_{2} I_{2}^{*}\right) X_{1}^{*}-\frac{\epsilon_{1} u_{1}^{*} X_{1}^{*}}{1+\zeta_{1} X_{1}^{*}}, \\
\frac{d X_{2}^{*}}{d t} & =p \Lambda+\theta X_{1}^{*}-d X_{2}^{*}+q \gamma R^{*}-\left\{\beta_{1}\left(1+\omega_{1} \beta_{1}^{\prime}\right) I_{1}^{*}+\beta_{2}\left(1+\omega_{2} \beta_{2}^{\prime}\right) I_{2}^{*}\right\} X_{2}^{*}-\frac{\epsilon_{2} u_{2}^{*} X_{2}^{*}}{1+\zeta_{2} X_{2}^{*}}, \\
\frac{d I_{1}^{*}}{d t} & =\left(\beta_{1} I_{1}^{*}+\beta_{2} I_{2}^{*}\right) X_{1}^{*}+\left\{\beta_{1}\left(1+\omega_{1} \beta_{1}^{\prime}\right) I_{1}^{*}+\beta_{2}\left(1+\omega_{2} \beta_{2}^{\prime}\right) I_{2}^{*}\right\} X_{2}^{*}-(d+\delta+\eta) I_{1}^{*}, \\
\frac{d I_{2}^{*}}{d t} & =\eta I_{1}^{*}-(d+\delta) I_{2}^{*}-\alpha I_{2}^{*}-\frac{\epsilon_{3} u_{3}^{*} I_{2}^{*}}{1+\zeta_{3} I_{2}^{*}}, \\
\frac{d R^{*}}{d t} & =\alpha I_{2}^{*}-(\gamma+d) R^{*}+\frac{\epsilon_{1} u_{1}^{*} X_{1}^{*}}{1+\zeta_{1} X_{1}^{*}}+\frac{\epsilon_{2} u_{2}^{*} X_{2}^{*}}{1+\zeta_{2} X_{2}^{*}}+\frac{\epsilon_{3} u_{3}^{*} I_{2}^{*}}{1+\zeta_{3} I_{2}^{*}},
\end{aligned}
$$

with initial conditions: $X_{1}^{*}(0)>0, X_{2}^{*}(0)>0, I_{1}^{*}(0) \geq 0, I_{2}^{*}(0) \geq 0$ and $R^{*}(0) \geq 0$. The corresponding adjoint system is:

$$
\begin{aligned}
\frac{d \lambda_{1}}{d t} & =\lambda_{1}\left[d+\theta+\beta_{1} I_{1}^{*}+\beta_{2} I_{2}^{*}+\frac{\epsilon_{1} u_{1}^{*}}{\left(1+\zeta_{1} X_{1}^{*}\right)^{2}}\right]-\lambda_{2} \theta-\lambda_{3}\left(\beta_{1} I_{1}^{*}+\beta_{2} I_{2}^{*}\right)-\lambda_{5} \frac{\epsilon_{1} u_{1}^{*}}{\left(1+\zeta_{1} X_{1}^{*}\right)^{2}}, \\
\frac{d \lambda_{2}}{d t} & =\lambda_{2}\left[d+\beta_{1}\left(1+\omega_{1} \beta_{1}^{\prime}\right) I_{1}^{*}+\beta_{2}\left(1+\omega_{2} \beta_{2}^{\prime}\right) I_{2}^{*}+\frac{\epsilon_{2} u_{2}^{*}}{\left(1+\zeta_{2} X_{2}^{*}\right)^{2}}\right] \\
& -\lambda_{3}\left[\beta_{1}\left(1+\omega_{1} \beta_{1}^{\prime}\right) I_{1}^{*}+\beta_{2}\left(1+\omega_{2} \beta_{2}^{\prime}\right) I_{2}^{*}\right]-\lambda_{5} \frac{\epsilon_{2} u_{2}^{*}}{\left(1+\zeta_{2} X_{2}^{*}\right)^{2}}, \\
\frac{d \lambda_{3}}{d t} & =\lambda_{1} \beta_{1} X_{1}^{*}+\lambda_{2} \beta_{1}\left(1+\omega_{1} \beta_{1}^{\prime}\right) X_{2}^{*}-\lambda_{3}\left\{\beta_{1} X_{1}^{*}+\beta_{1}\left(1+\omega_{1} \beta_{1}^{\prime}\right) X_{2}^{*}-(d+\delta+\eta)\right\}-\lambda_{4} \eta, \\
\frac{d \lambda_{4}}{d t} & =-w_{1}+\lambda_{1} \beta_{2} X_{1}^{*}+\lambda_{2} \beta_{2}\left(1+\omega_{2} \beta_{2}^{\prime}\right) X_{2}^{*}-\lambda_{3}\left\{\beta_{2} X_{1}^{*}+\beta_{2}\left(1+\omega_{2} \beta_{2}^{\prime}\right) X_{2}^{*}\right\} \\
& +\lambda_{4}\left(d+\delta+\alpha+\frac{\epsilon_{3} u_{3}^{*}}{\left(1+\zeta_{3} I_{2}^{*}\right)^{2}}\right)-\lambda_{5}\left(\alpha+\frac{\epsilon_{3} u_{3}^{*}}{\left(1+\zeta_{3} I_{2}^{*}\right)^{2}}\right), \\
\frac{d \lambda_{5}}{d t} & =-\lambda_{1}(1-q) \gamma-\lambda_{2} q \gamma+\lambda_{5}(d+\gamma),
\end{aligned}
$$


with transversality conditions $\lambda_{i}\left(T_{f}\right)=0$, for $i=1,2,3,4,5$ and $u_{1}^{*}, u_{2}^{*}$ and $u_{3}^{*}$ are same as (9).

\section{REFERENCES}

1. Holmes J.C. Parasites as threats to biodiversity in shrinking ecosystems. Biodivers. Conserv. 1996. V. 5. P. 975-983.

2. Rigby M.C., More, Y. Life-history trade-offs and immune defenses. In: Evolutionary Biology of Host-Parasite Relationships: Theory Meets Reality. Eds. Poulin R., Morand S., Skorping A. Amsterdam: Elsevier Science, 2000. P. 129-142.

3. Beck M.A., Levander O.A. Host nutritional status and its effect on a viral pathogen. $J$. Infect. Dis. 2000. V. 182. P. S93-S96.

4. Khan R.A. Parasitism in marine fish after chronic exposure to petroleum hydrocarbons in the laboratory and to the Exxon Valdez oil spill. Bull. Environ. Contam. Toxicol. 1990. V. 44. P. 759-763.

5. Harvell C.D., Kim K., Burkholder J.M., Colwell R.R., Epstein P.R., Grimes D.J., Hofmann E.E., Lipp E.K., Osterhaus A.D., Overstreet R.M. et al. Emerging marine diseases-climate links and anthropogenic factors. Science. 1999. V. 285. P. 1505-1510. doi: 10.1126/science.285.5433.1505

6. Scott M.E. The impact of infection and disease on animal populations: implications for conservation biology. Conserv. Biol. 1980. V. 2. P. 40-56.

7. Blanford S., Thomas M.B, Pugh C., Pell J.K. Temperature checks the Red Queen? Resistance and virulence in a fluctuating environment. Ecol. Lett. 2003. V. 6. P. 2-5. doi: 10.1046/j.1461-0248.2003.00387.x

8. Dubey B. A model for the effect of pollutant on human population dependent on a resource with environmental and health policy. J. Biol. Syst. 2010. V. 18. No. 03. P. 571-592.

9. Shukla J., Agrawal A., Dubey B., Sinha P. Existence and survival of two competing species in a polluted environment: a mathematical model. J. Biol. Syst. 2001. V. 9. No. 02. P. 89-103. doi: 10.1142/S0218339001000359

10. Shukla J., Misra A., Chandra P. Mathematical modelling of the survival of a biological species in polluted water bodies. Differ. Equ. Dyn. Syst. 2007. V. 15. P. 209-230.

11. Mandal P.K. Dioxin: a review of its environmental effects and its aryl hydrocarbon receptor biology. J. Comp. Physiol. B. 2005. V. 175. No. 4. P. 221-230.

12. Brook R. Cardiovascular effects of air pollution. Clin. Sci. 2008. V. 115. P. 175-187.

13. Nawahda A., Yamashita K., Ohara T., Kurokawa J., Yamaji K. Evaluation of premature mortality caused by exposure to $P M_{2.5}$ and ozone in East Asia: 2000, 2005, 2020. Water Air Soil Pollut. 2012. V. 223. No. 6. P. 3445-3459. doi: $10.1007 / \mathrm{s} 11270-012-1123-7$

14. Pope C.A. 3rd, Burnett R.T., Thun M.J., Calle E.E., Krewski D., Ito K., Thurston G.D. Lung cancer, cardiopulmonary mortality, and long-term exposure to fine particulate air pollution. JAMA 2002. V. 287. No. 9. P. 1132-1141. doi: 10.1001/jama.287.9.1132

15. Javan, S., Rahdar, S., Miri, M., Djahed B., Kazemian H., Fakhri Y., Eslami H., Fallahzadeh R.A., Gholizadeh A., Taghavi M. Modeling of the $P M_{10}$ pollutant health effects in a semi-arid area: a case study in Zabol, Iran. Model. Earth Syst. Environ. 2021. V. 7. P. 455-463. doi: 10.1007/s40808-020-00874-y

16. Laumbach R.J., Kipen H.M. Respiratory health effects of air pollution: update on biomass smoke and traffic pollution. J. Allergy Clin. Immunol. 2012. V. 129. No. 1. P. 3-11.

17. Salvi S. Health effects of ambient air pollution in children. Paediatr. Respir. Rev. 2007. V. 8. No. 4. P. 275-280.

18. Schell L. M., Gallo M.V., Denham M., Ravenscroft J. Effects of pollution on human growth and development: an introduction. J. Physiol. Anthropol. 2006. V. 25. No. 1. P. 103-112. doi: 10.2114/jpa2.25.103 
19. van Rossem L., Rifas-Shiman S.L., Melly S.J., Kloog I., Luttmann-Gibson H., Zanobetti A., Coull B.A., Schwartz J.D., Mittleman M.A., Oken E. et al. Prenatal air pollution exposure and newborn blood pressure. Environ. Health Perspect. 2015. V. 123 No. 4. P. 353-359. doi: 10.1289/ehp.1307419

20. Schwarzman M.R., Wilson M.P. New science for chemicals policy. Science. 2009. V. 326. No. 5956. P. 1065.

21. Richardson S.D. Disinfection by-products and other emerging contaminants in drinking water. Trends Anal. Chem. 2003. V. 22. No. 10. P. 666-684.

22. Hamidin N., Yu, Q.J., Connell, D.W. Human health risk assessment of chlorinated disinfection by-products in drinking water using a probabilistic approach. Water Res. 2008. V. 42. No. 13. P. 3263-3274.

23. Al-Mikhlafi A. S. Groundwater quality of yemen volcanic terrain and their geological and geochemical controls. Arab. J. Geosci. 2010. V. 3. No. 2. P. 193-205.

24. Ahmad S.A., Sayed M., Barua S., Khan M.H., Faruquee M.H., Jalil A., Hadi S.A., Talukder H.K. Arsenic in drinking water and pregnancy outcomes. Environ. Health Perspect. 2001. V. 109. No. 6. P. 629. doi: 10.1289/ehp.01109629

25. Waller K., Swan S.H., DeLorenze G., Hopkins B. Trihalomethanes in drinking water and spontaneous abortion. Epidemiology 1998. V. 9. No. 2. P. 134-140.

26. Collie A.C. Pharmaceutical contaminants in potable water: potential concerns for pregnant women and children. EcoHealth. 2007. V. 4. No. 2. P. 164-171.

27. Hertz-Picciotto I., Park H.Y., Dostal M., Kocan A., Trnovec T., Sram R. Prenatal exposures to persistent and non-persistent organic compounds and effects on immune system development. Basic Clin. Pharmacol. Toxicol. 2008. V. 102. No. 2. P. 146-154. doi: 10.1111/j.1742-7843.2007.00190.x

28. Grandjean P., Bellinger D., Bergman A., Cordier S., Davey-Smith G., Eskenazi B., Gee D., Gray K., Hanson M., van den Hazel P. et al. The faroes statement: human health effects of developmental exposure to chemicals in our environment. Basic Clin. Pharmacol. Toxicol. 2008. V. 102. No. 2. P. 73-75. doi: 10.1111/j.1742-7843.2007.00114.x

29. Raqib R., Ahmed S., Sultana R., Wagatsuma Y., Mondal D., Hoque A.M., Nermell B., Yunus M., Roy S., Persson L.A. et al. Effects of in utero arsenic exposure on child immunity and morbidity in rural Bangladesh. Toxicol. Lett. 2009. V. 185. No. 3. P. 197-202. doi: 10.1016/j.toxlet.2009.01.001

30. McMichael A.J., Woodruff R.E., Hales S. Climate change and human health: present and future risks. Lancet. 2006. V. 367. No. 9513. P. 859-869.

31. Patz J. A., Graczyk T.K., Geller N., Vittor A.Y. Effects of environmental change on emerging parasitic diseases. Int. J. Parasitol. 2000. V. 30. No. 12. P. 1395-1405. doi: 10.1016/s0020-7519(00)00141-7

32. Lipp E. K., Huq A., Colwell R.R. Effects of global climate on infectious disease: the cholera model. Clin. Microbiol. Rev. 2002. V. 15. No 4. P. 757-770.

33. Dolschak K., Gartner K., Berger T.W. The impact of rising temperatures on water balance and phenology of European beech (Fagus sylvatica L.) stands. Model. Earth Syst. Environ. 2019. V. 5. P. 1347-1363. doi: $10.1007 / \mathrm{s} 40808-019-00602-1$

34. Stemn E., Kumi-Boateng B. Modelling of land surface temperature changes as determinant of urban heat island and risk of heat-related conditions in the Wassa West Mining Area of Ghana. Model. Earth Syst. Environ. 2020. V. 6. P. 1727-1740. doi: 10.1007/s40808-020-00786-X

35. Ayub S., Akhter G., Ashraf A., Iqbal M. Snow and glacier melt runoff simulation under variable altitudes and climate scenarios in Gilgit River Basin, Karakoram region. Model. Earth Syst. Environ. 2020. V. 6. P. 1607-1618. doi: 10.1007/s40808-020-00777-y 
36. Devi S., Mishra R.P. A mathematical model to see the effects of increasing environmental temperature on plant-pollinator interactions. Model. Earth Syst. Environ. 2020. V. 6. P. 1315--1329. doi: 10.1007/s40808-020-00763-4

37. Traoré A.N., Mulaudzi K., Chari G.J., Foord S.H., Mudau L.S., Barnard T.G., Potgieter $\mathrm{N}$. The impact of human activities on microbial quality of rivers in the Vhembe District, South Africa. Int. J. Environ. Res. Public Health. 2016. V. 13. No. 8. P. 817. doi: 10.3390/ijerph13080817

38. Roumagnac P., Weill F.X., Dolecek C., Baker S., Brisse S., Chinh N.T., Le T.A., Acosta C.J., Farrar J., Dougan G. et al. Evolutionary history of Salmonella typhi. Science 2006. V. 314. No. 5803. P. 1301-1304. doi: 10.1126/science.1134933

39. Riggs M.M., Sethi A.K., Zabarsky T.F., Eckstein E.C., Jump R.L., Donskey C.J. Asymptomatic Carriers Are a Potential Source for Transmission of Epidemic and Nonepidemic Clostridium difficile Strains among Long-Term Care Facility Residents. Clinical Infectious Diseases. 2007. V. 45. No. 8. P. 992-998. doi: 10.1086/521854.

40. Lafferty K.D., Holt R.D. How should environmental stress affect the population dynamics of disease? Ecol. Lett. 2003. V. 6. No. 7. P. 654-664.

41. Hale J.K. Theory of functional Differential Equations. Heidelberg: Springer-Verlag, 1977.

42. Van den Driessche P., Watmough J. Reproduction numbers and sub-threshold endemic equilibria for compartmental models of disease transmission. Mathematical Biosciences 2002. V. 180. No. 1. P. 29-48.

43. Arriola L., Hyman J. Lecture notes on forward and adjoint sensitivity analysis with applications in Dynamical Systems, Linear Algebra and Optimisation. Mathematical and Theoretical Biology Institute, 2005.

44. Mathematical Approaches for Emerging and Reemerging Infectious Diseases: Models, Methods, and Theory. Eds. Castillo-Chavéz C., Blower S., van den Driessche P., Kirschner D., Yakubu A.-A. Springer, 2002. (The IMA Volumes in Mathematics and its Applications, V. 126). doi: 10.1007/978-1-4613-0065-6

45. LaSalle J. The stability of dynamical systems. Philadelphia: SIAM, 1976. (CBMS-NSF Regional Conference Series in Applied Mathematics). doi: 10.1137/1.9781611970432

46. Castillo-Chavéz, C., Song, B. Dynamical models of tuberculosis and their applications. Math. Biosci. Eng. 2004. V. 1. P. 361-404.

47. Saha S., Samanta G.P. Modelling and optimal control of HIV/AIDS prevention through PrEP and limited treatment. Physica A: Statistical Mechanics and Its Applications. 2019. V. 516. P. 280-307. doi: 10.1016/j.physa.2018.10.033

48. Saha S., Samanta G.P. Dynamics of an epidemic model with impact of toxins. Physica A: Statistical Mechanics and Its Applications. 2019. V. 527. Article No. 121152. doi: 10.1016/j.physa.2019.121152

49. Saha S., Samanta G.P., Nieto J.J. Epidemic model of COVID-19 outbreak by inducing behavioural response in population. Nonlinear Dyn. 2020. V. 102. P. 455-487. doi: 10.1007/s11071-020-05896-w

50. Gaff H., Schaefer E. Optimal control applied to vaccination and treatment strategies for various epidemiological models. Mathematical Biosciences and Engineering. 2009. V. 6. No. 3. P. 469-492.

51. Kassa S., Ouhinou A. The impact of self-protective measures in the optimal interventions for controlling infectious diseases of human population. Journal of Mathematical Biology. 2015. V. 70. No. 1-2. P. 213-236.

52. Joshi H., Lenhart S., Li M., Wang L. Optimal control methods applied to disease models. Contemporary Mathematics. 2006. V. 410. P. 187-208. doi: 10.1090/conm/410/07728

53. Behncke H. Optimal control of deterministic epidemics. Optimal Control Applications and 
Methods. 2000. V. 21. No. 6. P. 269-285.

54. Castilho, C. Optimal control of an epidemic through educational campaigns. Electronic Journal of Differential Equations. 2006. V. 125. P. 1-11.

55. Hove-Musekwa S.D., Nyabadza F., Chiyaka C., Das P., Tripathi A., Mukandavire Z. Modelling and analysis of the effects of malnutrition in the spread of cholera. Math. Comput. Model. 2011. V. 53. No. 9. P. 1583-1595. doi: $10.1016 / \mathrm{j} . \mathrm{mcm} .2010 .11 .060$

56. Vardavas R., Blower S. The emergence of HIV transmitted resistance in Botswana: when will the WHO detection threshold be exceeded? PLOS ONE. 2007. V. 2. No. 1. Article No. e152. doi: 10.1371/journal.pone.0000152

57. Donnell D., Baeten J.M., Kiarie J., Thomas K.K., Stevens W., Cohen C.R., McIntyre J., Lingappa J.R., Celum C. Heterosexual HIV-1 transmission after initiation of antiretroviral therapy: a prospective cohort analysis. Lancet 2010. V. 375. P. 2092-2098. doi: 10.1016/S0140-6736(10)60705-2

58. Collins O., Govinder K. Incorporating heterogeneity into the transmission dynamics of a waterborne disease model. J. Theor. Biol. 2014. V. 356. P. 133-143.

59. Tien J. H., Earn D.J. Multiple transmission pathways and disease dynamics in a waterborne pathogen model. Bull. Math. Biol. 2010. V. 72. No. 6. P. 1506-1533.

60. Kirk D. Optimal control theory: an introduction. Dover Publications, 2012. (Dover Books on Electrical Engineering)

61. Coddington E., Levinson N. Theory of ordinary differential equations. Tata McGraw-Hill Education, 1955.

62. Fleming W., Rishel R. Deterministic and stochastic optimal control. New York: Springer, 1975. (Applications of Mathematics, V. 1)

63. Pontryagin L. Mathematical theory of optimal processes. CRC Press, 1987.

Accepted 12.01.2021.

Revised 23.06.2021.

Published 07.07.2021. 